\title{
DEVELOPMENT AND VALIDATION OF A COMPUTATIONAL MULTIBODY MODEL OF THE ELBOW JOINT
}

\author{
A THESIS IN \\ Mechanical Engineering \\ Presented to the Faculty of the University \\ of Missouri-Kansas City in partial fulfillment of \\ the requirements for the degree \\ MASTER OF SCIENCE
}

\author{
by \\ MD MUNSUR RAHMAN
}

B.S., Bangladesh University of Engineering and Technology, 2009

Kansas City, Missouri

2013 
(C) 2013

MD MUNSUR RAHMAN

ALL RIGHTS RESERVED 


\title{
DEVELOPMENT AND VALIDATION OF A COMPUTATIONAL MULTIBODY MODEL OF THE ELBOW JOINT
}

\author{
MD MUNSUR RAHMAN, Candidate for the Master of Science Degree \\ University of Missouri - Kansas City, 2013
}

\begin{abstract}
Computational multibody models of the elbow joint can provide a powerful tool to study joint biomechanics, examine muscle and ligament function, soft tissue loading, and the effects of joint trauma. Such models can reduce the cost of expensive experimental testing and can predict some parameters that are difficult to investigate experimentally, such as forces within ligaments and contact forces between cartilage covered bones. These parameters can assist surgeons and other investigators to develop better treatments for elbow injuries and thereby increase patient care. Biomechanical computational models of the elbow exist in the literature, but these models are typically limited in their applicability by artificially constraining the joint (e.g. modeling the elbow as a hinge joint), prescribing specific kinematics, simplifying ligament characteristics or ignoring cartilage geometries. The purpose of this thesis was to develop anatomically correct subject specific computational multibody models of elbow joints and validate these models against experimental data. In these models, the joints were constrained by three-dimensional deformable contacts between articulating geometries, passive muscle loading, and multiple bundles of non-linear ligaments wrapped around the bones.
\end{abstract}


In this approach, three-dimensional bone geometries for the model were constructed from volume images generated by computed tomography (CT) scans obtained from cadaver elbows. The ligaments and triceps tendon were modeled as spring-damper elements with non-linear stiffness. Articular cartilage was represented as uniform thickness solids covering the articulating bone surfaces. Finally, the model was validated by placing the cadaver elbows in a mechanical testing apparatus and comparing predicted kinematics and triceps tendon forces to experimentally measured values. A small improvement in predicted kinematics was observed compared to experimental values when the lateral ulnar collateral and annular ligament were wrapped around the bone. Some reductions of RMS error were also observed when a non-linear toe region was modeled in the ligament compared to models that had only a linear force-displacement relationship. None of these changes were statistically significant (ANOVA $p$-value was greater than 0.05 ). 
The undersigned, appointed by the Dean of School of Computing and Engineering, have examined the thesis titled " Development and Validation of a Computational Multibody Model of the Elbow Joint," presented by Md Munsur Rahman, candidate for the Master of Science in Mechanical Engineering degree, and thereby certify that in their opinion it is worthy of acceptance.

$\underline{\text { Supervisory Committee }}$

Trent M. Guess, Ph.D

Associate Professor

School of Computing and Engineering

Ganesh Thiagarajan, Ph.D

Associate Professor

School of Computing and Engineering

Gregory W. King, Ph.D

Assistant Professor

School of Computing and Engineering 


\section{TABLE OF CONTENTS}

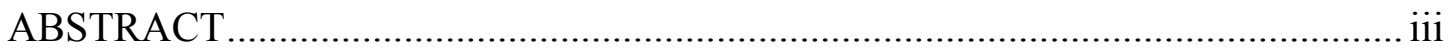

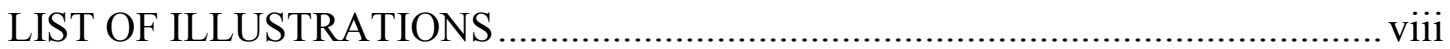

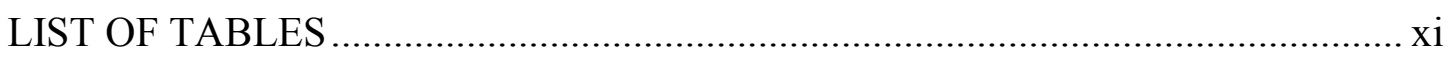

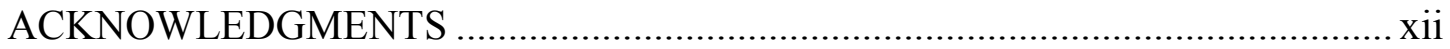

Chapter

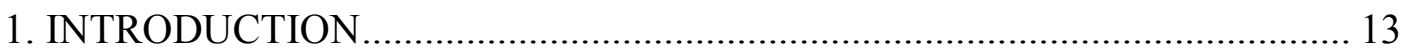

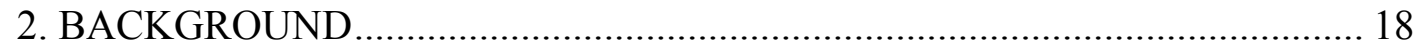

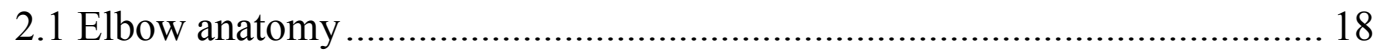

2.1.1 Bone anatomy ................................................................... 19

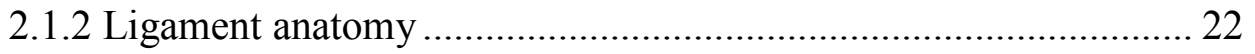

2.1.3 Muscle anatomy................................................................. 25

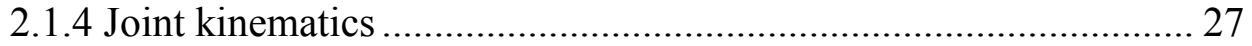

3. METHODS AND MATERIALS .............................................................. 30

3.1 Cadaver elbow measurements and testing ...................................... 30

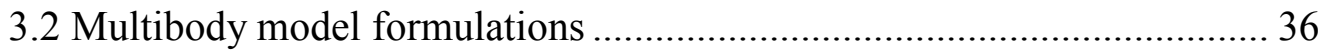

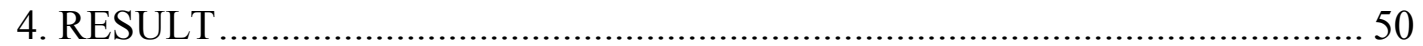

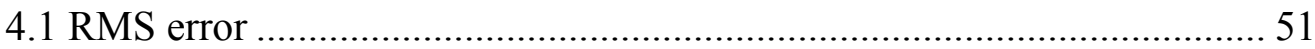

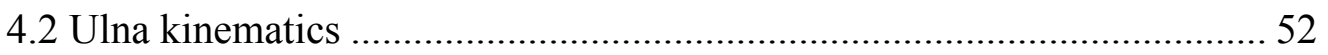

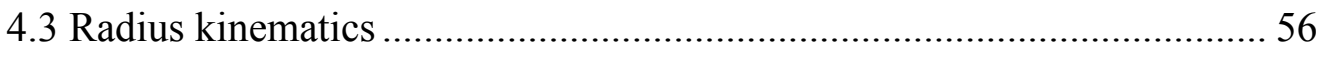


4.3 Triceps tendon forces.

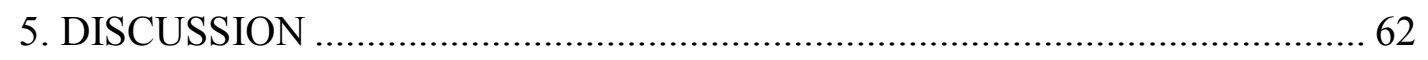

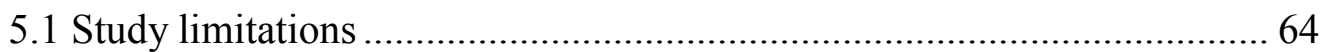

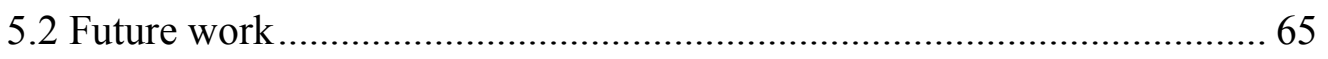

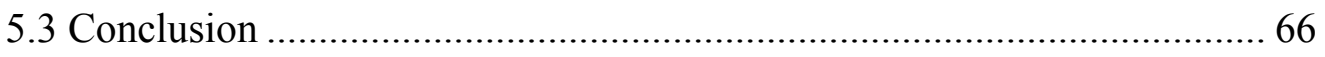

Appendix

A. KINEMATICS COMPARISON FOR SPECIMEN 2 AND 3 ........................... 67

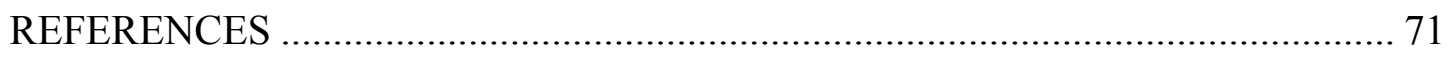

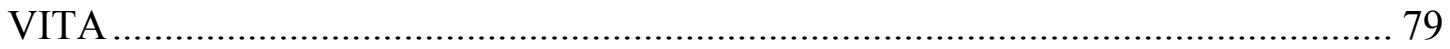




\section{LIST OF ILLUSTRATIONS}

Figure

Page

2.1. (A) The view of entire upper extremity and (B) the articulations of elbow joint. Source: Ferreira (2011). 18

2.2. Osteology of the elbow joint. Anterior (left) and a posterior (right) view of the right elbow is shown. Source: Netter and Hansen (2003).

2.3. Osteology of ulna and radius. Anterior (left) and lateral view of ulna (right). Source: Tate (2012).

2.4. Medial (left) and Lateral (right) collateral ligament complex. Source: Morrey (2000).................................................................. 23

2.5. Interosseous membrane of the forearm. Source: Fisk (2007). ........................... 25

2.6. View of the four major muscles crossing the elbow joint. Source: ("Identify the muscles crossing the elbow joint," 2013)....

2.7. (A) The flexion-extension and (B) pronation supination view of forearm. Right arm is shown. Source: Ferreira (2011).

3.1. (a) The disarticulated radius for specimen 1. The radius was cut from the distal radioulnar joint and the ulna was cemented directly to the cup. (b) A ten hole steel dynamic compression plate was used to constrain the ulna for specimens 2 and 3.

3.2. Triceps tendon attachment to the load cell and motion applied by the mechanical tester. 33

3.3. Laxity test to measure the zero load length. 34

3.4. Auto threshold segmentation of CT images to isolate the bone geometries in 3D Slicer.

3.5. Three-dimensional bone geometries a) before and b) after post processing in Geomagic Studio.

3.6. The experimental cadaver elbow setup in the mechanical tester. (b) Multibody model of the elbow in Adams. The approximate position of the humerus, ulna, and radius coordinate system are also indicated. The definition of the local elbow coordinate systems were obtained from Ferreira et al. (2011) , and Morrey and Chao (1976).

3.7. The force-displacement relationship for the central bundle of the medial collateral ligament (cMCL) anterior part. The 
measured zero-load length of the cMCL was $17.2 \mathrm{~mm}$ and the stiffness coefficient in the linear region was $24.1 \mathrm{~N} / \mathrm{mm}$.

3.8. Interosseous membrane and distal radioulnar ligaments in the model. Right limb shown.

3.9. Wrapping of the LUCL and annular ligament around the bone. Also shown is the point-curve constraints and parallel connection between two spheres of the LUCL and annular ligaments.

3.10. Approximate position and orientation of the elbow joint coordinate system. Source: Ferreira (2011).

4.1. Superior-inferior (S-I) displacement of the ulna coordinate system relative to the humerus coordinates for specimen 1 .

4.2. Anterior-posterior (A-P) displacement of the ulna coordinate system relative to the humerus coordinates for specimen 1 .....

4.3. Medial-lateral (M-L) displacement of the ulna coordinate system relative to the humerus coordinates for specimen 1 .

4.4. Ulna internal-external (I-E) rotation relative to the humerus coordinates for specimen 1

4.5. Ulna adduction-abduction (AD-AB duction) relative to the humerus coordinates for specimen 1 . 55

4.6. Ulna flexion-extension (F-E) relative to the humerus coordinates for specimen 1 .

4.7. S-I displacement of the radius coordinate system relative to the humerus coordinates for specimen 1 .

4.8. A-P displacement of the radius coordinate system relative to the humerus coordinates for specimen 1

4.9. M-L displacement of the radius coordinate system relative to the humerus coordinates for specimen 1 .

4.10. Radius I-E rotation relative to the humerus coordinates for specimen 1 .

4.11. Radius I-E rotation relative to the humerus coordinates for specimen 1 .

4.12. Radius $A D-A B$ duction relative to the humerus coordinates for specimen 1

4.13. Comparison of triceps tendon force for specimen 1.

A.1. Measured and predicted displacement and rotation of the ulna coordinate system relative to the humerus coordinate system for specimen 2 . 
A.2 Measured and predicted displacement and rotation of the radius coordinate system relative to the humerus coordinate system for specimen 2 .

A.3. Measured and predicted displacement and rotation of the ulna coordinate system relative to the humerus coordinate system for specimen 3 .

A.4. Measured and predicted displacement and rotation of the radius coordinate system relative to the humerus coordinate system for specimen 3 . 


\section{LIST OF TABLES}

Table

Page

3.1. Information regarding each cadaver elbow used in this study. .......................... 31

3.2. Elbow flexion angle during movement. ........................................................... 35

3.3. Ligament modeling parameters. (Fisk \& Wayne, 2009; Regan et al., 1991; Spratley \& Wayne, 2011).......................................................... 42

3.4. Stiffness parameters for interosseous membrane. (Fisk, 2007; Peck et al., 2000; Schuind et al., 1991)........................................................ 43

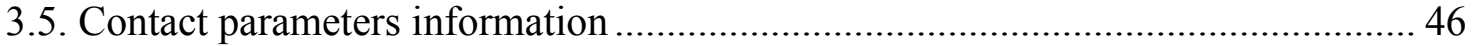

4.1. RMS error between predicted and measured kinematics and triceps tendon forces. Errors are shown for each ligament condition. The top values for each axis are shown in bold. 


\section{ACKNOWLEDGMENTS}

I would like to thank the School of Medicine, University of Missouri-Kansas City (UMKC), for funding this research.

I would like to acknowledge many people who have helped and provided support for me during this project. To begin with my advisor Dr. Guess, who has provided a great opportunity to work in the Musculoskeletal Biomechanics Research Laboratory and enabled me to have a fulfilling research experience. He opened the door to a subject that I have become passionate about. His guidance, support, and wisdom have also been indispensable. I would also like to acknowledge Dr. Akin Cil for his support and guidance to successfully finish this research. I would like to thank my advisory committee members Dr. Thiagarajan and Dr. King for taking the time and effort to serve on my committee. I want to thank my lab partners Mohammad Kia, Antonis Stylianou, and Katherine Bloemker, Yunkai Lu for helping troubleshoot problems.

I would also like to thank my wife Mashruba for her unwavering support and care throughout this process. 


\section{CHAPTER 1}

\section{INTRODUCTION}

The human elbow joint is a unique joint that produces the complex motion of the forearm for hand positioning and allows humans to accomplish numerous significant activities in their daily life that makes them distinct from other mammals (Gonzalez, Hutchins, Barr, \& Abraham, 1996).Unfortunately, this most important joint of the upper extremity (Morrey, 2000) has been recognized as the second most commonly dislocated joint in adults (de Haan et al., 2011). Although not as common as in the knee joint, osteoarthritis of the elbow can cause severe pain in the joint, loss of joint mobility, and can be a cause of entire upper limb disability (Degreef \& De Smet, 2011). The enervating nature of osteoarthritis is well established, but the underlying reasons of this chronic disease are not completely understood. Furthermore, fracture, tennis elbow, tendinitis, bursitis, and motion impingement can be significantly debilitating for the elbow joint. Additionally, the high frequency of dislocation (Wiesel \& Delahay, 2010), complexity of posttraumatic instability (Ring \& Jupiter, 2000), and operative complications associate with joint trauma (Ring \& Jupiter, 2000) have made the elbow joint an important focus of research (Fisk, 2007).

Detailed knowledge of the in vivo loading of elbow structures is important to understand the biomechanical causes associated with joint degeneration and injuries and to find suitable treatment. Prediction of joint and tissue level loading during elbow activities has a great potential to significantly improve orthopaedic repair. In addition, better understanding of in vivo mechanical loads has good indications regarding the development 
and progression of osteoarthritis and thereby reduces the cost of treatment (Guess, Thiagarajan, Kia, \& Mishra, 2010). Likewise, knowledge of in vivo ligament and tendon forces of elbow joint would provide valuable insight regarding joint stability and injury mechanism. But currently, measuring the in vivo tendon and ligament forces and cartilage contact pressures of the elbow joint during various activities is not possible.

Computational multibody models can be a potential tool to predict tendon, ligament and contact forces (Cohen, Henry, McCarthy, Mow, \& Ateshian, 2003; Giddings, Beaupre, Whalen, \& Carter, 2000; Hirokawa, 1991; Kwak, Blankevoort, \& Ateshian, 2000; Lemay \& Crago, 1996; Shelburne, Pandy, Anderson, \& Torry, 2004; Wismans, Veldpaus, Janssen, Huson, \& Struben, 1980). These models could provide valuable understanding to the in vivo loading environment of the elbow joint and enhance our comprehension of elbow mechanics and tissue interactions during dynamic activities (Guess, 2012). Therefore, computational multibody models of the elbow could be a valuable tool to improve the diagnosis, treatment and rehabilitation of post-traumatic injuries of the elbow joint. Many researchers have used these model to investigate muscle contribution to joint moment (Arnold \& Delp, 2001; Gonzalez et al., 1996; Hutchins, Gonzalez, \& Barr, 1993; Lemay \& Crago, 1996; Murray, Delp, \& Buchanan, 1995; van der Helm, 1994a) and body segment motion (Anderson \& Pandy, 2001; Gonzalez, Abraham, Barr, \& Buchanan, 1999; Nagano, Komura, Yoshioka, \& Fukashiro, 2005; Peck, Langenbach, \& Hannam, 2000; van der Helm, 1994b). A validated model can be used as a potential biomechanical tool for patient-specific preoperative planning, computer-aided surgery, and computer-aided rehabilitation (Chao, Armiger, Yoshida, Lim, \& Haraguchi, 2007; Fernandez \& Pandy, 2006; Fisk \& Wayne, 2009; 
Holzbaur, Murray, \& Delp, 2005; Kwak et al., 2000; Woo, Debski, Wong, Yagi, \& Tarinelli, 1999).

Two main tools have been used in biomechanics for developing computational models: finite element analysis (FEA) (Giddings et al., 2000; Li, Gil, Kanamori, \& Woo, 1999; van der Helm, 1994b; Wu, Dong, Smutz, \& Schopper, 2003) and multibody dynamics (MBD) (Anderson \& Pandy, 2001; Barker, Kirtley, \& Ratanapinunchai, 1997; Chaudhari \& Andriacchi, 2006; Cohen et al., 2003; Freund \& Takala, 2001; Gonzalez, Andritsos, Barr, \& Abraham, 1993; Gonzalez et al., 1996; Hirokawa, 1991; Iwasaki et al., 1998; Kwak et al., 2000; Lemay \& Crago, 1996; Li et al., 1999; Liacouras \& Wayne, 2007; Lin et al., 2005; Morey-Klapsing, Arampatzis, \& Bruggemann, 2005; Nagano et al., 2005; Peck et al., 2000; Piazza \& Delp, 2001; Raikova, 1992, 1996; Shelburne et al., 2004; Triolo, Werner, \& Kirsch, 2001; Wismans et al., 1980). FEA has the ability to predict the stress and strain within tissue in articulating contacts. The models are based on the concepts of continuum mechanics and contain many equations and unknown variables. As a result, finite element models take extensive amounts of time for both development and simulation. Therefore, FEA is not an efficient option in body level dynamic simulation (Guess \& Stylianou, 2012). On the other hand, multibody modeling uses rigid body dynamics algorithms where the models have fewer unknown variables. MBD simulations are computationally efficient for dynamic simulation compared to FEA. As a result, for the situations where calculating bone deformation or stress-strain computations are not the choice, multibody modeling could be an appealing option. Many researchers have applied multibody models for specific applications such as predicting joint stability, joint contact areas and pressure, ligament functions, muscle contributions to joint moments, body segment motion, and menisci effect in the knee 
(Donahue, Hull, Rashid, \& Jacobs, 2002; Ferreira, King, \& Johnson, 2011; Fisk \& Wayne, 2009; Guess, 2012; Guess, Liu, Bhashyam, \& Thiagarajan, 2013; Guess et al., 2010; Stylianou, Guess, \& Cook, 2012; Zielinska \& Donahue, 2006).

Numerous studies of computational modeling have been developed to investigate joint behavior (Buchanan, Delp, \& Solbeck, 1998; Fisk \& Wayne, 2009; Garner \& Pandy, 2001; Gonzalez et al., 1999; Gonzalez et al., 1996; Holzbaur et al., 2005; Kwak et al., 2000; Lemay \& Crago, 1996; Raikova, 1992; Schuind et al., 1991; Spratley \& Wayne, 2011; Triolo et al., 2001). Gonzalez et al. (1996) developed a computational elbow model to investigate the elbow joint movement, relationship among muscle excitation patterns, and to determine the effects of forearm and elbow position on the recruitment of individual muscles during ballistic movements. Holzbaur et al. (2005) developed a three-dimensional model of the upper extremity that comprises all the major muscles of the upper limb and provides accurate estimation of muscle moment arms. Lemay and Crago (1996) developed a dynamic skeletal model of the elbow joint where the model movements were produced by activation of Hilltype muscle models and were capable of simulating elbow and wrist flexion-extension, and radial-ulnar deviation movement. However, these models have assumed the joint structure to have idealized joint motion (e.g. hinge joint) rather than true anatomical joint motion constrained by ligament force and cartilage contact. Although in some circumstance such simplification would be helpful for our understanding of joint kinematics and muscle functions, it is not always appropriate to assume a human joint as a generalized mechanical joint (Benham, Wright, \& Bibb, 2001). These presumptions decrease the validity of model results, and prevent investigation of ligament function and joint laxity (Fisk, 2007). Fewer biomechanical models of the elbow joint have investigated the ligamentous constraints, 
articular surface contact, and muscle loading effect on joint stability, but have not included wrapping of ligaments around bone, the non-linear ligament 'toe' region, and articular cartilage contribution (Fisk \& Wayne, 2009; Spratley \& Wayne, 2011). Irrespective of any modeling assumption, some models have been limited by model validation (Chao et al., 2007; Delp \& Loan, 1995; Fernandez \& Pandy, 2006; Kwak et al., 2000; Woo et al., 1999). Model predicted results should be compared with experimental data before a computational model can be used as a predictive and meaningful tool (Fisk, 2007).

The main purpose of this thesis is to develop an anatomically correct computational elbow joint model that includes representation of articular cartilage. The elbow joint is constrained by three-dimensional contact between articular bone geometries, triceps tendon loading, and multiple ligament bundles having a non-linear toe region. Wrapping of the lateral ulnar collateral and annular ligaments around the bone are also considered. This study has examined the effects of articular contacts and different ligament loading on kinematics during elbow flexion-extension associated with forearm pronation-supination. The models have used easily accessible, well documented commercial software for developing the computational models. The models are validated by comparing the predicted humerus, radius, ulna kinematics and passive triceps tendon forces to identically loaded experimentally measured values. Developing anatomically correct elbow models in the multibody framework for incorporation in musculoskeletal models of the upper extremities is the overall goal of this research. 


\section{CHAPTER 2}

\section{BACKGROUND}

\subsection{Elbow anatomy}

Elbows play an important role for positioning and orientating the upper arm in threedimensional space. Careful examination of elbow anatomy is important to understand the significant influences of various components on joint structure and behavior. Anatomy of the elbow is relevant to all structures that accomplish and affect elbow motion such as bone, ligaments, and muscle. As the most important joint of the upper extremity (Alcid, Ahmad, \& Lee, 2004), the elbow joint is comprised of all three long bones of the arm; humerus, radius, and ulna (Fig. 2.1).

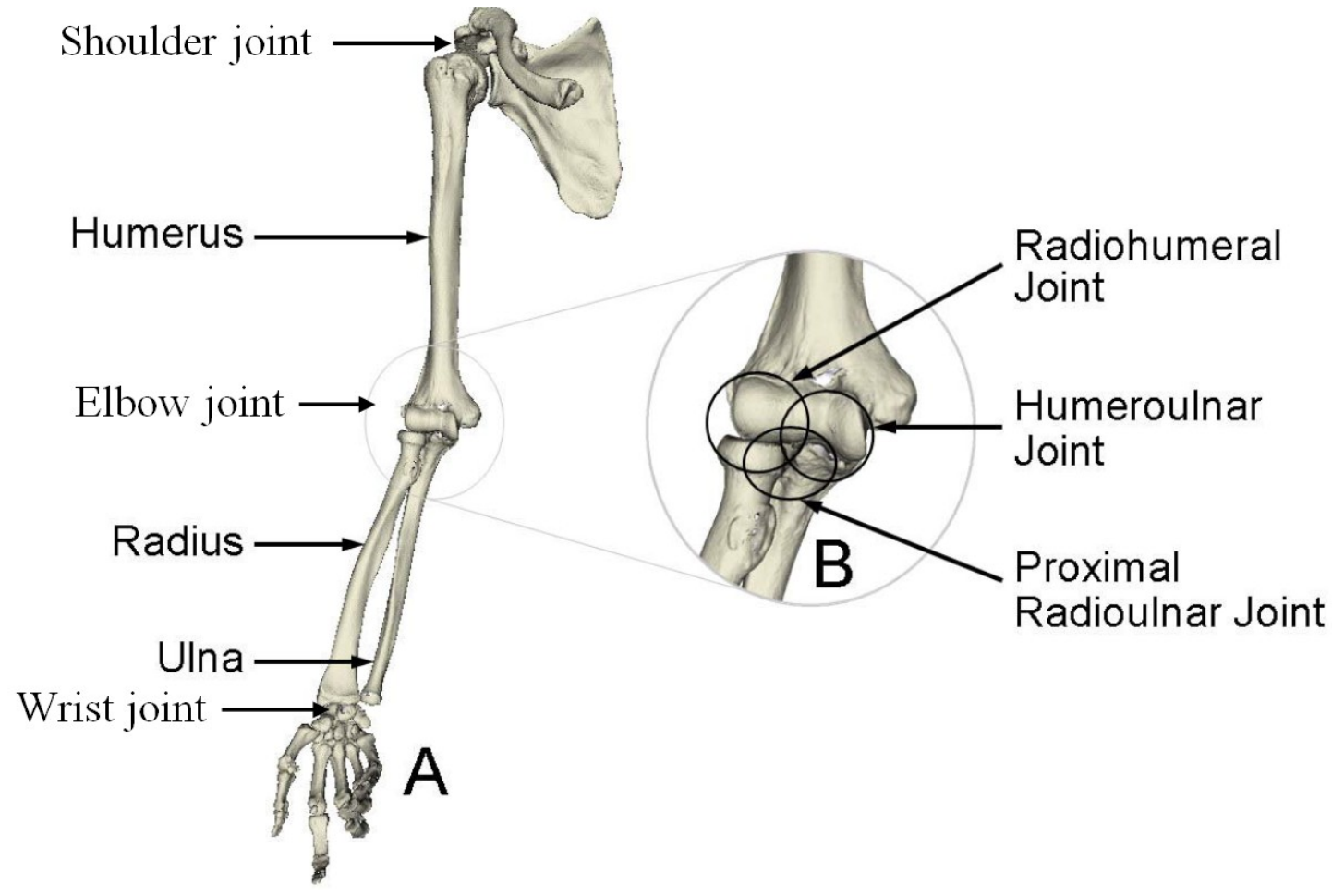

Figure 2.1. (A) The view of entire upper extremity and (B) the articulations of elbow joint. Source: Ferreira (2011). 


\subsubsection{Bone anatomy}

The humerus is the longest bone of the upper arm. It is associated with articulation of both the shoulder and elbow joint. The humeral head has a half spheroid shape that is covered with articular hyaline cartilage. The humerus shaft is cylindrical at the proximal end, and becomes wedge shaped distally. The distal humerus widens on both sides inferiorly and forms medial and lateral epicondyles (Fig. 2.2).

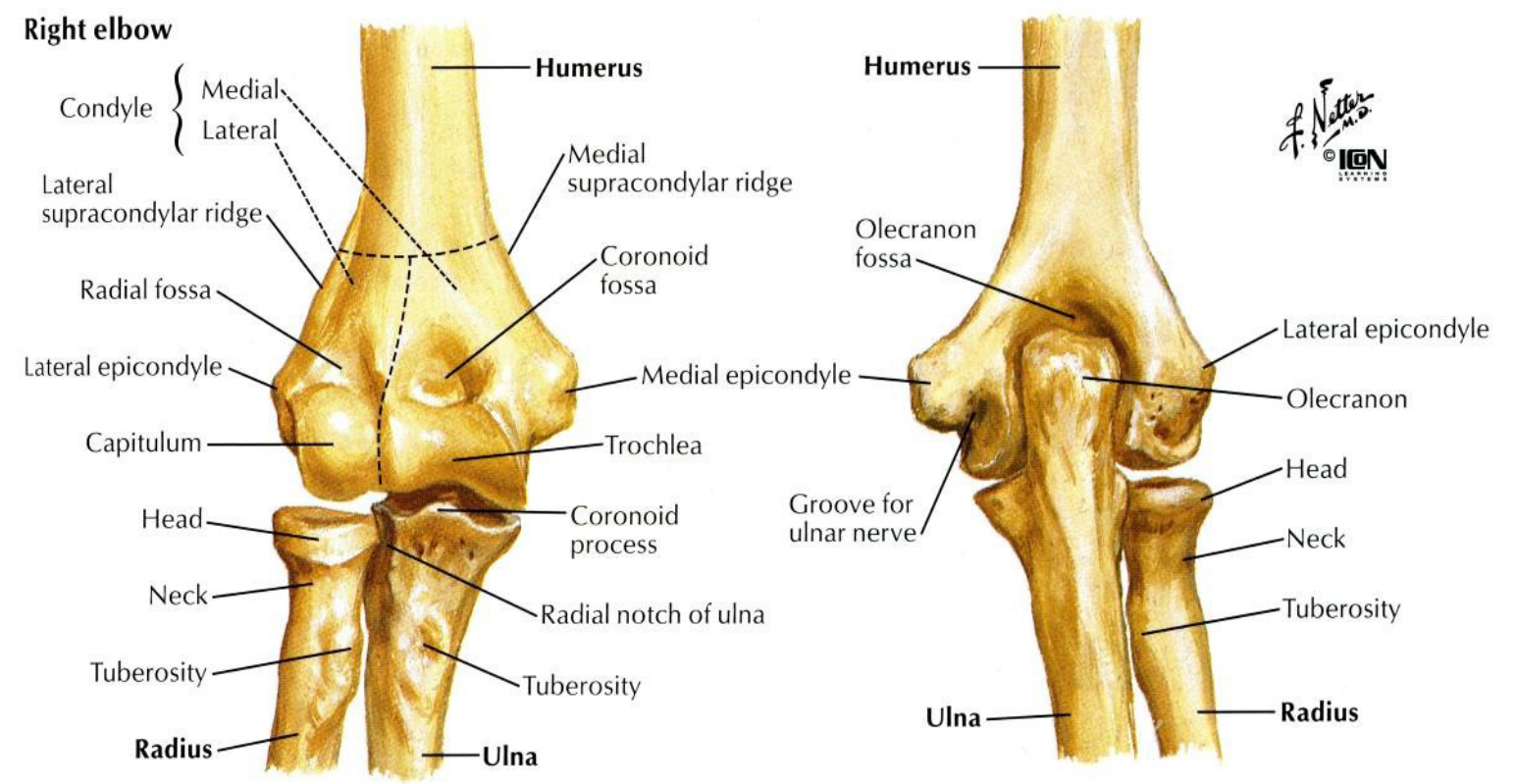

Figure 2.2. Osteology of the elbow joint. Anterior (left) and a posterior (right) view of the right elbow is shown. Source: Netter and Hansen (2003).

The epicondyles serve as the attachment sites for the medial and lateral collateral ligaments, as well as muscle attachment points for the hand and forearm. The distal surface of the humerus has a large but complex contour of hyaline cartilage, allowing articulation with both the ulna and radius. The distal humerus contains many intricate structures necessary for elbow function (e.g. elbow flexion-extension and forearm pronation- 
supination). Adjacent to the medial epicondyle is the spool-shaped surface of the trochlea. The trochlea surface is covered by $300^{\circ}$ of articular cartilage (Chuang, Wu, Lin, \& Lur, 2012; Morrey, 2000) which articulates with the greater sigmoid notch of the ulna. This surface has a circular cross-section in the sagittal plane and contains the trochlear sulcus at the center which provides an articular bearing surface for flexion-extension motion. The trochlear sulcus with the medial and lateral lips forms a track that keeps the greater sigmoid notch of the ulna centered. Lateral to the trochlea is the capitulum that has a nearly spherical structure. The capitulum is covered with articular cartilage by approximately $180^{\circ}$ (Ferreira, 2011) that allows articulation with the concave dish of the radial head and provides a bearing for both elbow flexion-extension and forearm pronation-supination. Two groove structures located superior to trochlear and capitulum are called the coronoid fossa and radial fossa respectively (Fig. 2.2). The depression region on the posterior distal humerus is the olecranon fossa that provides clearance of the ulna's olecranon at high elbow extension.

Structures of the proximal radius include several crucial features that are necessary for proper elbow operation. At its most proximal aspect is the cup-shaped structure called the radial head (Fig. 2.2). The axis of the radial head and the adjacent neck make a $15^{\circ}$ angle with the radius shaft (Morrey, 2000). The radial head is fully enveloped with articular cartilage and provides articulation with the capitulum to allow forearm rotation. The circumference of the radial head is also covered with hyaline cartilage by $240^{\circ}$ (Ferreira, 2011). This cartilage contributes to articulation with the lesser sigmoid notch of the proximal ulna and forms the proximal radioulnar joint. A bony outcropping at the distal radius is the radial tubercle that serves as the insertion site for biceps tendon (Morrey, 2000). 
The proximal ulna has some very significant structures for elbow function. Most superiorly, the ulna bone comes forward, approximating the form of a beak (Fig. 2.2). This beak is called the olecranon process. The anteriorly extended surface is called coronoid process that stays distally from the olecranon process. The olecranon and coronoid process fits into their corresponding fossae during full elbow flexion and extension. The cartilage enveloped region of the proximal ulna is divided into two regions; the lesser and greater sigmoid notch. The lesser sigmoid notch has $60-80^{\circ}$ of articular cartilage that articulates with the radial head and forms the proximal radioulnar joint (PRUJ) which provides forearm pronation-supination. The greater sigmoid notch generates articulations with the distal humerus where the guiding ridge fits into the track of the trochlear sulcus of the humerus.

Elbow function is also influenced by skeletal features distal to the joint. The diaphysis shafts of the ulna and radius area are triangular in cross section and run almost parallel to each other to the distal end when the forearm is supinated (Fig. 2.3). Interosseous margin areas are located at the medial and lateral boundaries of the supinated radius and ulna diaphysis respectively. The radius becomes tapered as it goes distally to its limit, but the ulna expands with triangular structure till it terminates at its distal head. At the distal end of the radius, the bony lateral extension is called the styloid process. The styloid process provides attachments for ligaments of the wrist. At the distal end of the ulna, the cartilage covered knoblike head articulates laterally with a notch on the radius (ulnar notch) and forms the distal radioulnar joint. This joint, together with the proximal radioular joint, provides articulation for forearm rotational motion. A medial styloid process located at the distal end of the ulna provides attachments for ligaments of the wrist. 


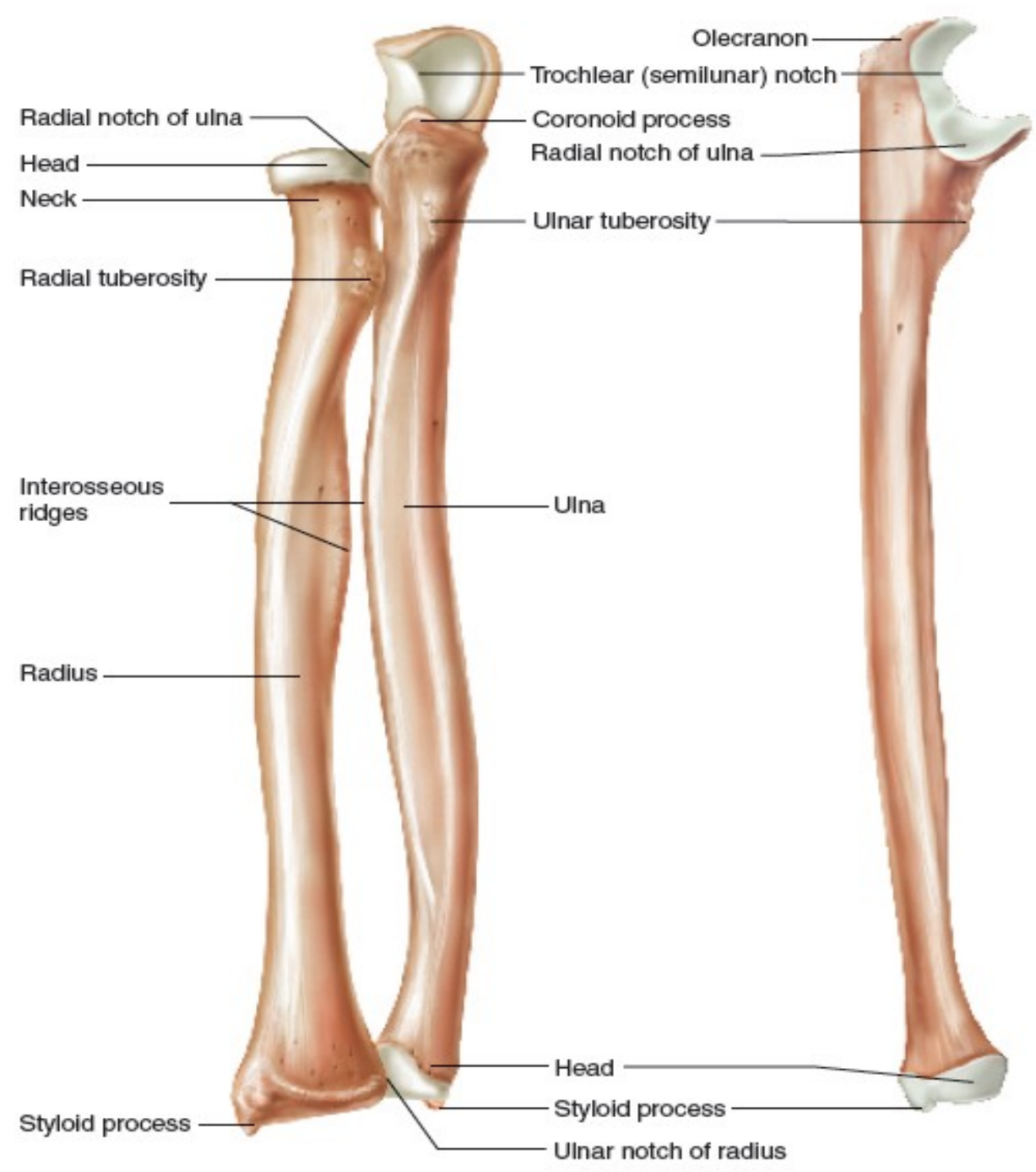

Figure 2.3. Osteology of ulna and radius. Anterior (left) and lateral view of ulna (right). Source: Tate (2012)

\subsubsection{Ligament anatomy}

The bony structures of the elbow joint are restrained and stabilized by other passive structures. Predominantly, the medial and lateral collateral ligaments are two major ligamentous structures that contribute to primary stabilization of the elbow. The forearm is largely stabilized by the interosseous membrane between the ulna and radius. Distal radioulnar ligaments, located at distal radioulnar joint, can also influence elbow behavior. 
The medial collateral ligament (MCL) complex consists of three segments: the anterior, posterior, and transverse bundles (Cohen \& Bruno, 2001; Fuss, 1991; Morrey \& An, 1985). The anterior bundle of the MCL originates from the medial epicondyle of the humerus and attaches to the sublime tubercle on the coronoid process of the ulna (Fig. 2.4). The posterior bundle also originates from the humerus medial epicondyle, however, its ulnar attachments are much broader. Though the attachment site is less defined, it generally inserts more distally onto the medial aspect of the olecranon. The anterior and posterior bundles of the medial collateral ligament have a substantial effect on joint stability in response to valgus movement. It also plays a significant role in limiting elbow flexion and extension. The transverse ligament bundle originates and attached to the ulna only, and so far has no known function (Morrey, 2000).
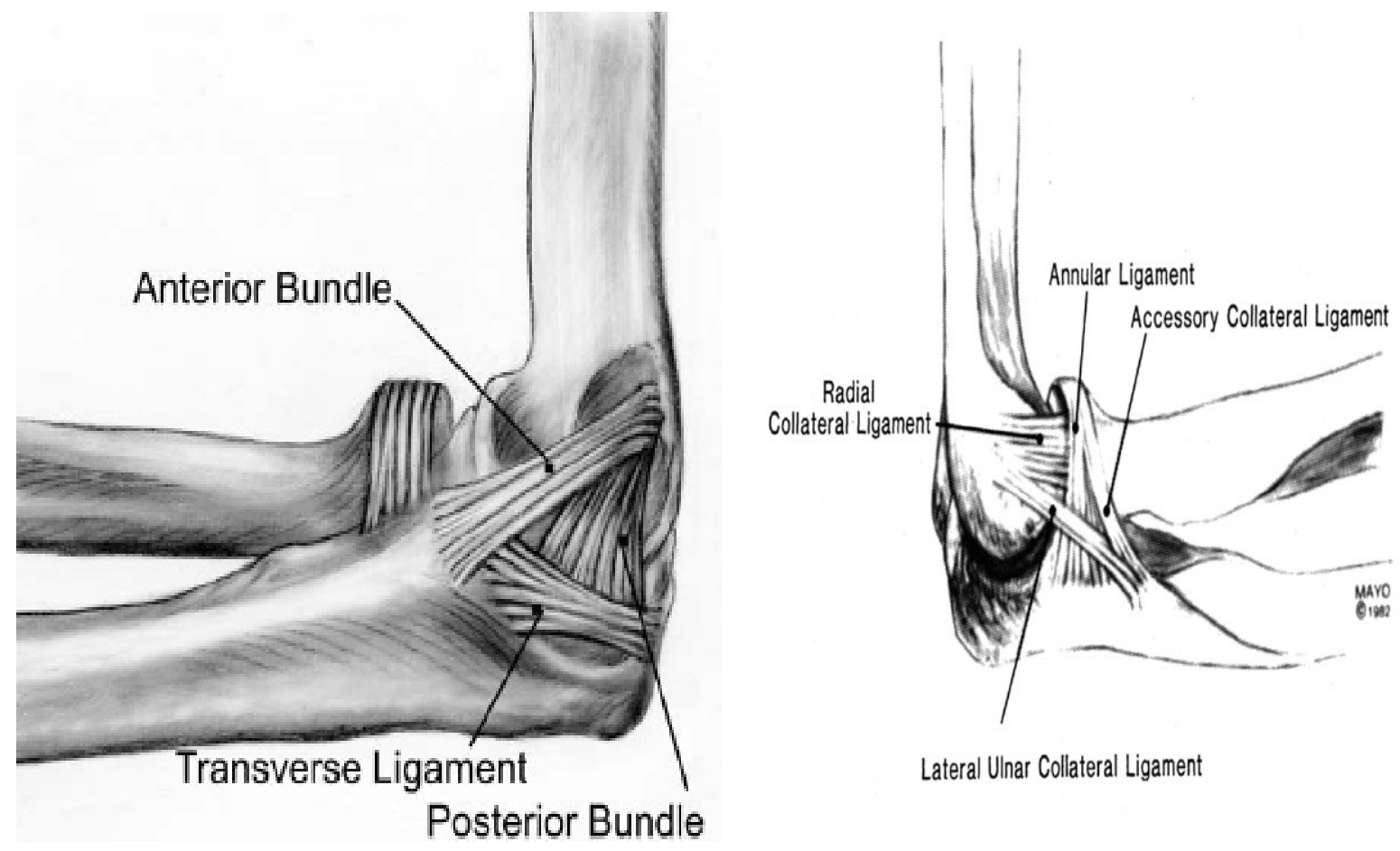

Lateral Ulnar Collateral Ligament

Figure 2.4. Medial (left) and Lateral (right) collateral ligament complex. Source: Morrey (2000). 
The lateral collateral ligament (LCL) typically includes four components (Morrey, 2000; Morrey \& An, 1985). The lateral ulnar collateral ligament (LUCL) originates from the lateral epicondyle of the humerus, and inserts in the crista supinatorum tubercle of the ulna and superficially blends with the annular ligament (Fig. 2.4). The ring-shaped annular ligament attaches to the anterior rim of the lesser sigmoid notch, wraps around approximately $80 \%$ of the radial head and attaches to the posterior rims of the lesser sigmoid notch. The radial collateral ligament $(\mathrm{RCL})$ originates from the lateral epicondyle of the humerus, fans out at its distal end, and blends with the lateral portion of the annular ligament. A variable accessory collateral ligament is sometimes described, which attaches to the crista supinatorum and blends proximally with the distal lateral rim of the annular ligament (Morrey, 2000).

Ligaments are viscoelastic (An, 2005), making the ligament mechanical characteristics dependent on the direction of load and also on loading rate. Each ligament bundle has fibers orientated in different directions according to the primary tensile force. The collateral ligaments are heterogeneous structures which are composed of a combination of collagen and elastin that provide stability of the ligament in various directions. The interosseous membrane (IOM) is the sturdy thin collagenous sheet that usually attaches on the interosseous borders of the radius and ulna (Fig. 2.5). It consists of several bands: proximal band, central band, accessory bands, and distal membranous band (McGinley \& Kozin, 2001; Skahen, Palmer, Werner, \& Fortino, 1997). Other than the proximal band, all interosseous membrane bands runs distally and medially from its radial origin to its ulnar insertion. The fiber bands make an average $21^{0}$ angle with the long axis of the ulna (Skahen et al., 1997). The proximal band is an oblique structure that attaches proximally to the ulna 
and distally to the radius. The central band shares the insertion with proximal band and is approximately twice as thick as other bands (Amis, Dowson, \& Wright, 1979). Several other bands are located inferior to the central band; distal to these bands are areas of membranous tissue. Researchers have suggested that the interosseous membrane also acts as a stabilizer of the distal radioulnar joint (Schuind et al., 1991).

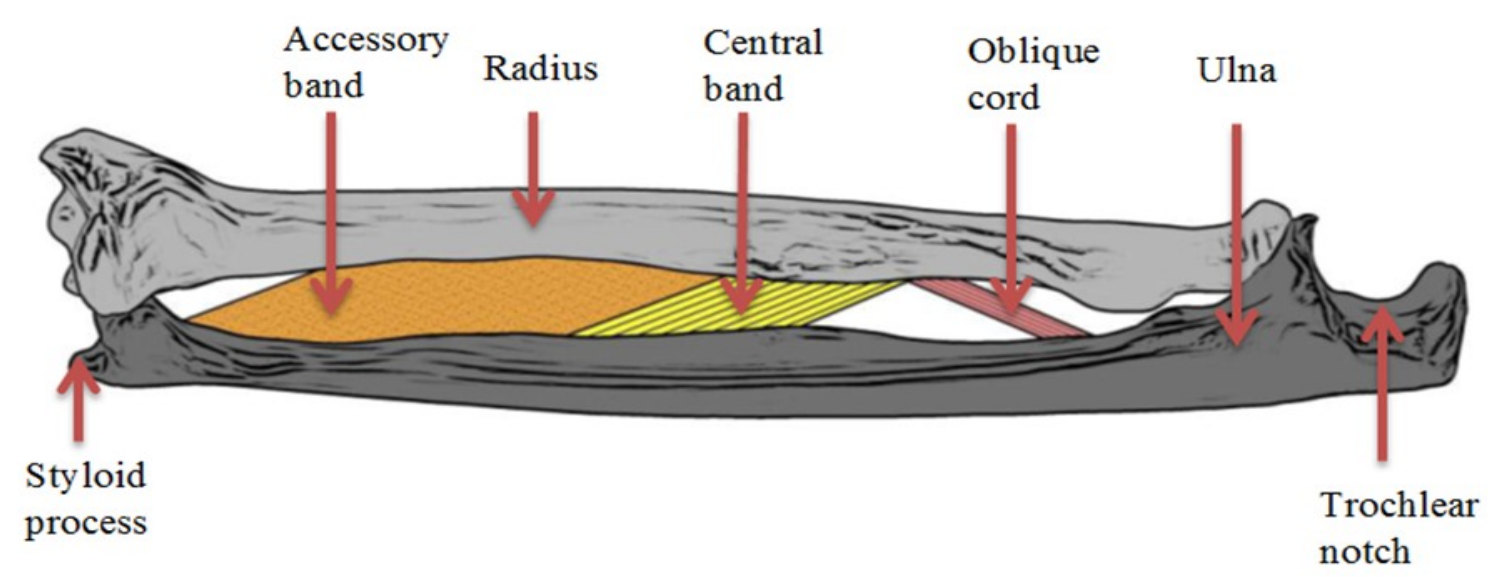

Figure 2.5. Interosseous membrane of the forearm. Source: Fisk (2007).

\subsubsection{Muscle anatomy}

Twenty four distinct muscles cross the elbow joint and these muscles originate from the distal humerus and insert on the forearm and hand (Morrey, 2000; Pigeon, Yahia, \& Feldman, 1996). These muscles produce flexion-extension, forearm pronation-supination, and flexion-extension of the wrist and fingers. Although all of the muscles are essential for proper elbow function, only a select subset of muscles that significantly influence elbow operation will be discussed here. 
Brachialis, biceps brachii, and brachioradialis are the three muscles that cross the elbow joint to generate flexion moment (Fig 2.6). The brachialis originates broadly on the anterior, distal half of the humerus and converges to insert more discretely on the ulnar tuberosity and base of the coronoid process. The biceps brachaii has two origins (its name is derived from the Latin word biceps which means "two heads") and positioned more superficially on the anterior aspect of the upper arm. The long head originates from the superior glenoid tubercle of the scapula and wraps around the humeral head and runs down to the intertubercular sulcus of the humerus. The short head originates from the apex of the coracoid process of scapula and blends with the long head approximately 7 centimeters proximal to the elbow to form a single tendon which inserts at the bicipital tuberosity of the proximal radius. The biceps brachii has a distinct large cross section and insertion on the medial aspect of the radius, as a result, the biceps works as a powerful forearm supinator. Particularly, when the forearm is supinated, biceps brachii works as a significant elbow flexor (Shiba et al., 1988). The brachioradialis originates from the lateral supracondylar ridge of the humerus and inserts distally at the radial styloid (Morrey, 2000). Brachioradialis has the longest moment arm of elbow flexion, but due to its small cross section, the brachioradialis works as the weakest of the three flexor muscles (Hotchkiss, An, Sowa, Basta, \& Weiland, 1989; Murray et al., 1995; Shiba et al., 1988). 


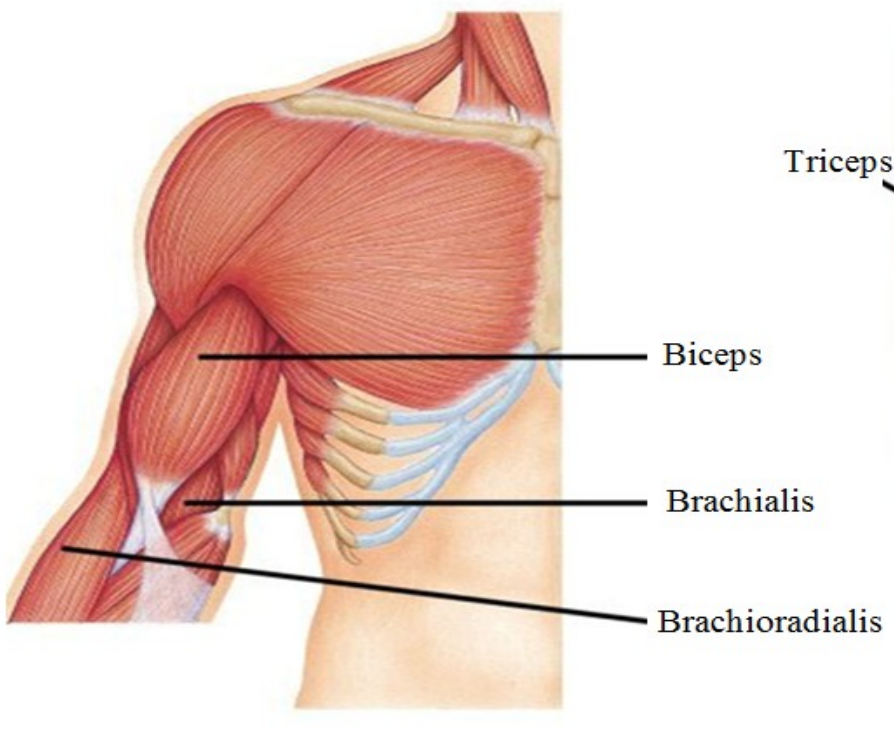

Anterior View

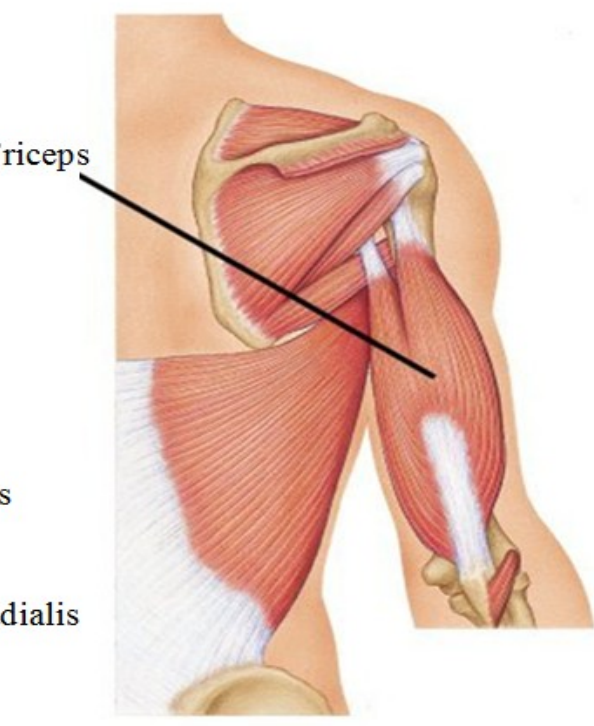

Posterior View

Figure 2.6. View of the four major muscles crossing the elbow joint. Source: ("Identify the muscles crossing the elbow joint," 2013)

The triceps is the only muscle that generates elbow extension moment. It has three heads, as its name implies. The long head has the most medial attachment and originates from the scapula at the infraglenoid tubercle. The lateral head originates on the lateral intermuscular septum and passes along a thin linear strip superior to the radial groove. The medial head has a broad origin and attaches to the posteromedial humeral shaft and medial intermuscular septum. All three heads begin to converge in the middle of the muscle and ultimately merge to form a single large tendon that inserts at the olecranon process of the ulna (Morrey, 2000).

\subsubsection{Joint kinematics}

The ulnohumeral, radiohumeral, proximal radioulnar, and distal radioulnar joint are described as a trochoginglymoid joint of upper extremities (Morrey, 2000) that jointly 
construct two distinct forms of motion (flexion-extension and pronation-supination). The flexion and extension motion is primarily produce by the ulnohumeral joint. The motion axis for flexion-extension is defined as an axis through the centers of the capitellum and the trochlear sulcus of the humerus (Currier, 1972). The full flexion range for normal subjects is approximately from $0^{\circ}$ (full extension) to $145^{\circ}$ (full flexion) (Figure 2.7A) (Morrey, 2000). Sometimes the hyperextension obtained from a subject is indicated by a negative flexion angle. The actual flexion range for an individual can be affected by many reasons such as prior disease or trauma, the bulk of soft tissue presence, and the ligamentous laxity or looseness.

Forearm pronation-supination is generated by the incorporation of the radiohumeral, proximal radioulna, and distal radioulnar articulation. To produce this motion, the ulna remains stationary and the radius pronates and supinates around it. Rather than circling the whole radius about the ulna, the distal radius encircles the distal ulna and the proximal radius pivots about its own center on the capitulum surface. For a normal subject, the attainable forearm ration range is about $150^{\circ}-160^{\circ}$ (Figure 2.7B) (Morrey, 2000), but it may vary depending on subject joint condition.

In addition to the above mentioned principal motions, the forearm bones exhibit other motion patterns. Along with rotation, the radius also moves proximally with pronation and distally with supination in the sagittal plane (Morrey, 2000). The proximal ulna rotates a few angles with respect to humerus and pronation-supination also causes the ulna to rotate internally and externally. 

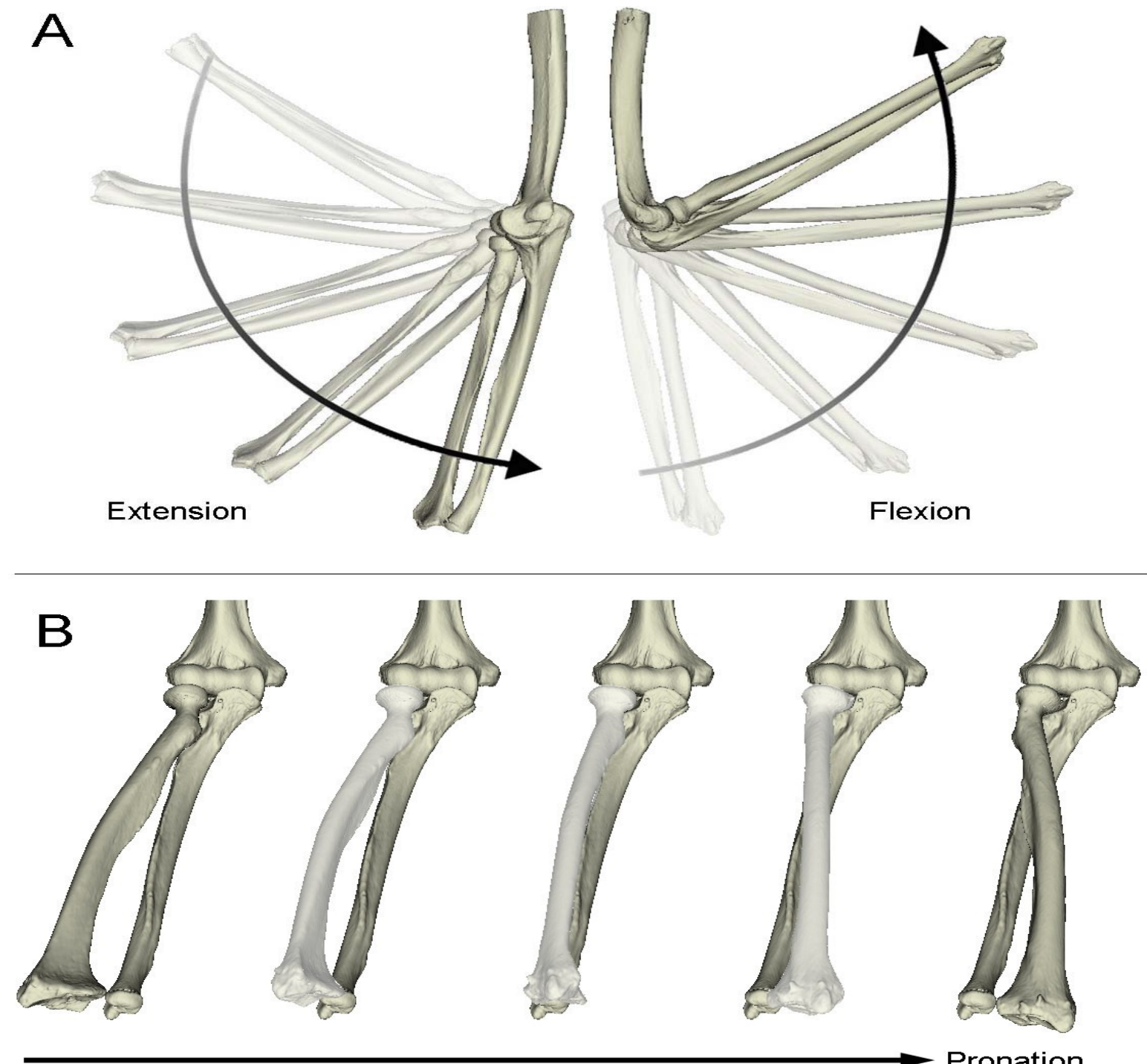

Supination

Figure 2.7. (A) The flexion-extension and (B) pronation supination view of forearm. Right arm is shown. Source: Ferreira (2011). 


\section{CHAPTER 3}

\section{METHODS AND MATERIALS}

\subsection{Cadaver elbow measurements and testing}

Three fresh frozen cadaver elbow specimens were used for this study (Table 3.1). The specimens were thawed at room temperature for 24 hours before collecting medical images. The elbow donors had never been diagnosed with major elbow diseases and the elbows appeared normal and intact during visual inspection. Two elbows were imaged with computed tomography (CT) scans and one was imaged with magnetic resonance imaging (MRI). The entire arm was scanned to obtain the complete bone lengths. CT scans of the elbows were taken to create three mutually perpendicular imaging sequences using Syngo CT (Siemens, Siemens medical solutions, PA) 20108 version software with 8 allocated bits and fine resolution scan. The parameters used for the CT scan imaging were: slice thickness of $1.5 \mathrm{~mm}$, imaging frequency $63.68 \mathrm{~Hz}$, spacing between slices $2 \mathrm{~mm}$, and group lengths 192 . MRIs were attained using a Siemens $1.5 \mathrm{~T}$ machine with a narrow field fine resolution setting. The parameters used for MRI were: TR:13.64, TE:6.82, image resolution 512 x 512, slice thickness $1.5 \mathrm{~mm}$, and spacing between slices $1.875 \mathrm{~mm}$. Before imaging, one custom made ABS plastic "localizer" was rigidly attached with titanium screws to each bone segment (humerus, ulna and radius) after limited incisions through the skin and soft tissues with the help of a shoulder and elbow fellowship trained orthopaedic surgeon. During the incisions, much care was taken to save the joint capsule and ligaments. Every localizer had two perpendicular tubes that were packed with Vaseline to assist in global coordinate registration 
later in the experiment (Stylianou et al., 2012). Following medical imaging, the elbows were dissected by the orthopedic surgeon. Keeping the joint capsule, ligaments, and triceps tendon intact, all other tissue was removed from the bone.

Table 3.1. Information regarding each cadaver elbow used in this study.

\begin{tabular}{l|c|c|c|c|c}
\hline & $\begin{array}{c}\text { Age at death } \\
\text { (years) }\end{array}$ & Gender & Right or Left & Height (in) & Weight (lbs) \\
\hline Specimen \#1 & 61 & Male & Left & 68 & 160 \\
\hline Specimen \#2 & 42 & Male & Right & 72 & 270 \\
\hline Specimen \#3 & 44 & Female & Left & 60 & 93 \\
\hline
\end{tabular}

After dissection, the elbows were mounted in a dynamic bi-axial mechanical tester (Bose 3510-AT). The humeral head was cemented inside a cylinder that was attached by a 1DOF hinge joint to the top ram of the mechanical tester. The top ram of the mechanical tester could only move in the vertical direction. The distal radius was cut about $8 \mathrm{~cm}$ from its distal end and disarticulated from the distal radioulnar joint, keeping the interosseous membrane partially intact (Fig. 3.1(a)) for specimen 1. The ulna was then constrained by cementing it into a cup that was connected to the bottom ram of the mechanical tester through a universal joint. The bottom ram of the mechanical tester could only rotate about a vertical axis. Rather than disarticulating the distal radioulnar joint for specimens 2 and 3, a 10 hole steel dynamic compression plate (Stryker, Mahwah, NJ) was used to constrain the distal ulna. Four titanium screws were applied through the holes of the plate to the ulna while the rest of the plate was cemented into the cup (Fig. 3.1(b)). 

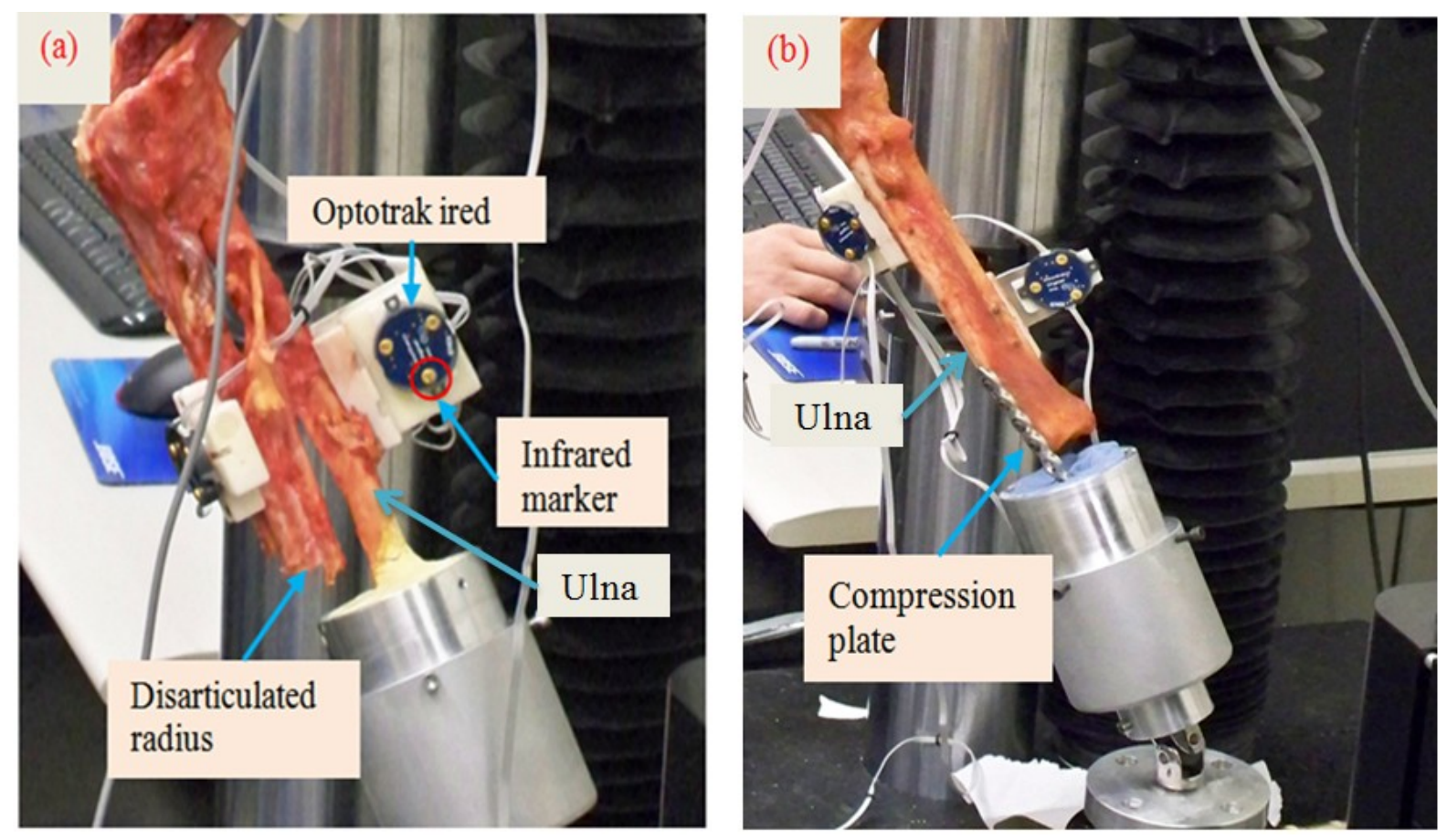

Figure 3.1. (a) The disarticulated radius for specimen 1. The radius was cut from the distal radioulnar joint and the ulna was cemented directly to the cup. (b) A ten hole steel dynamic compression plate was used to constrain the ulna for specimens 2 and 3 .

The radius had no extra mechanical constraint for all specimens. A $100 \mathrm{~N}$ load cell was rigidly attached to the humerus cylinder to measure force in the triceps tendon. The triceps tendon was threaded with a suture and the suture was attached to the load cell with the help of a threaded nut and bolt (Fig. 3.2). After attaching the arm to the testing machine, three rigid-body marker ireds (each containing three infrared markers (Fig. 3.1(a))) were firmly attached to the humerus, radius, and ulna. The humerus cylinder also had a marker ired added to it to measure the top ram movement and to aid in computational model alignment. A 3-camera Optotrak Certus motion Capture system (Northern Digital Inc,waterloo, Ontario, Canada) was used to track the motion of each bone segment during experimental testing. 


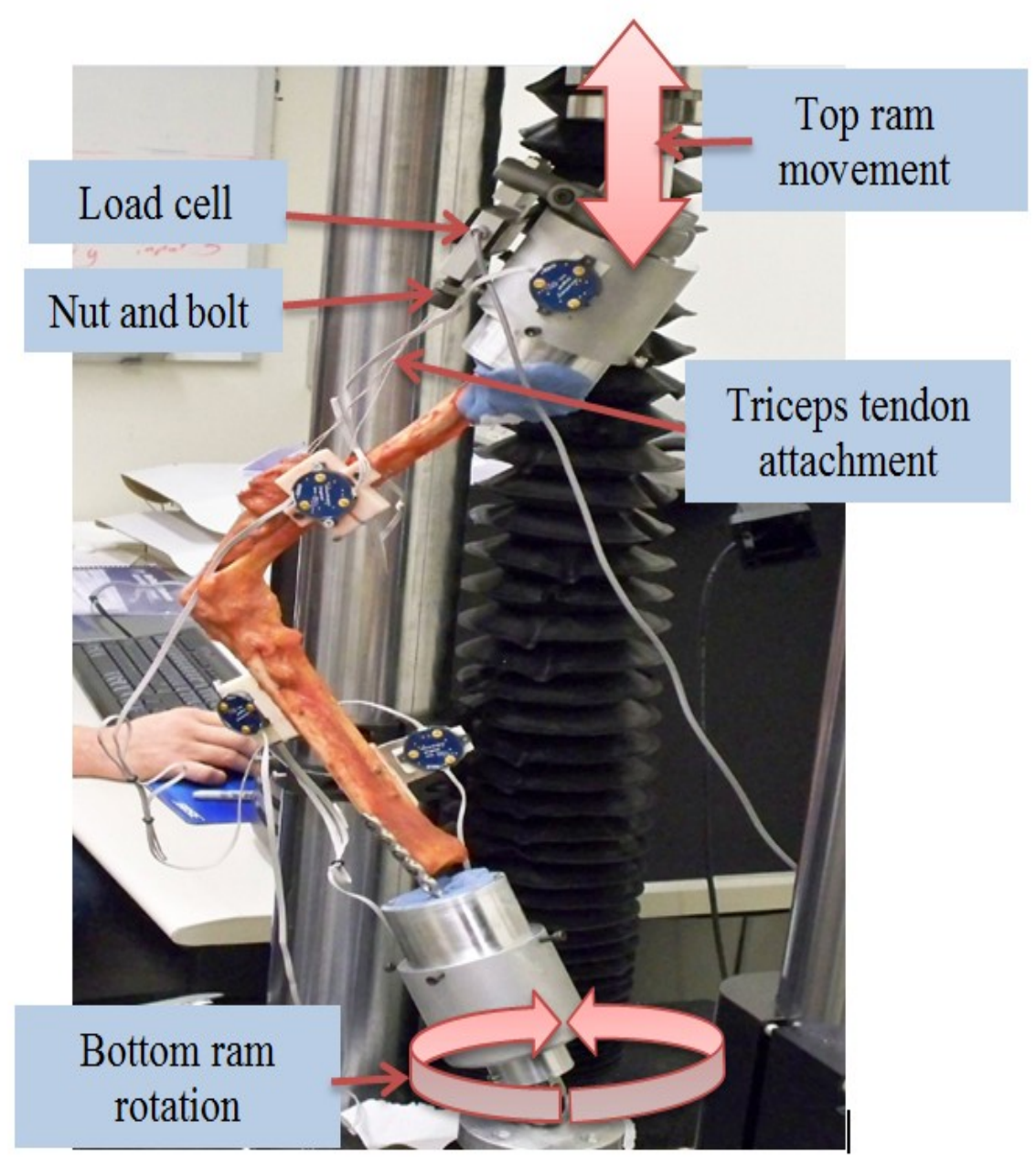

Figure 3.2. Triceps tendon attachment to the load cell and motion applied by the mechanical tester.

Before the experimental trials began, a laxity test was performed to calculate ligament bundle zero-load lengths (the lengths at which ligament bundles first become taut). To accomplish the laxity test, the humerus was held in a fixed position (Fig. 3.3) while the ulna and radius were manually moved through their full range of motion with minimal force applied (as judged by the experimenter) (Guess et al., 2013). The kinematic envelope of motion (KEM) was measured from corresponding bone segments by using the attached 
Optotrak markers and camera system throughout this process. The zero-load length for each ligament was then determined by calculating the maximum straight-line distance between insertion and origin sites of the individual ligament throughout the range of motion and then multiplying by a correction factor. A correction factor of 0.80 was applied to each ligament bundle (Bloemker, Guess, Maletsky, \& Dodd, 2012). The purpose of the correction factor was to reduce the error inadvertently introduced by the experimenter during the laxity test when a small amount of force was applied to each ligament when the experimenter moves the joint throughout its range of motion.

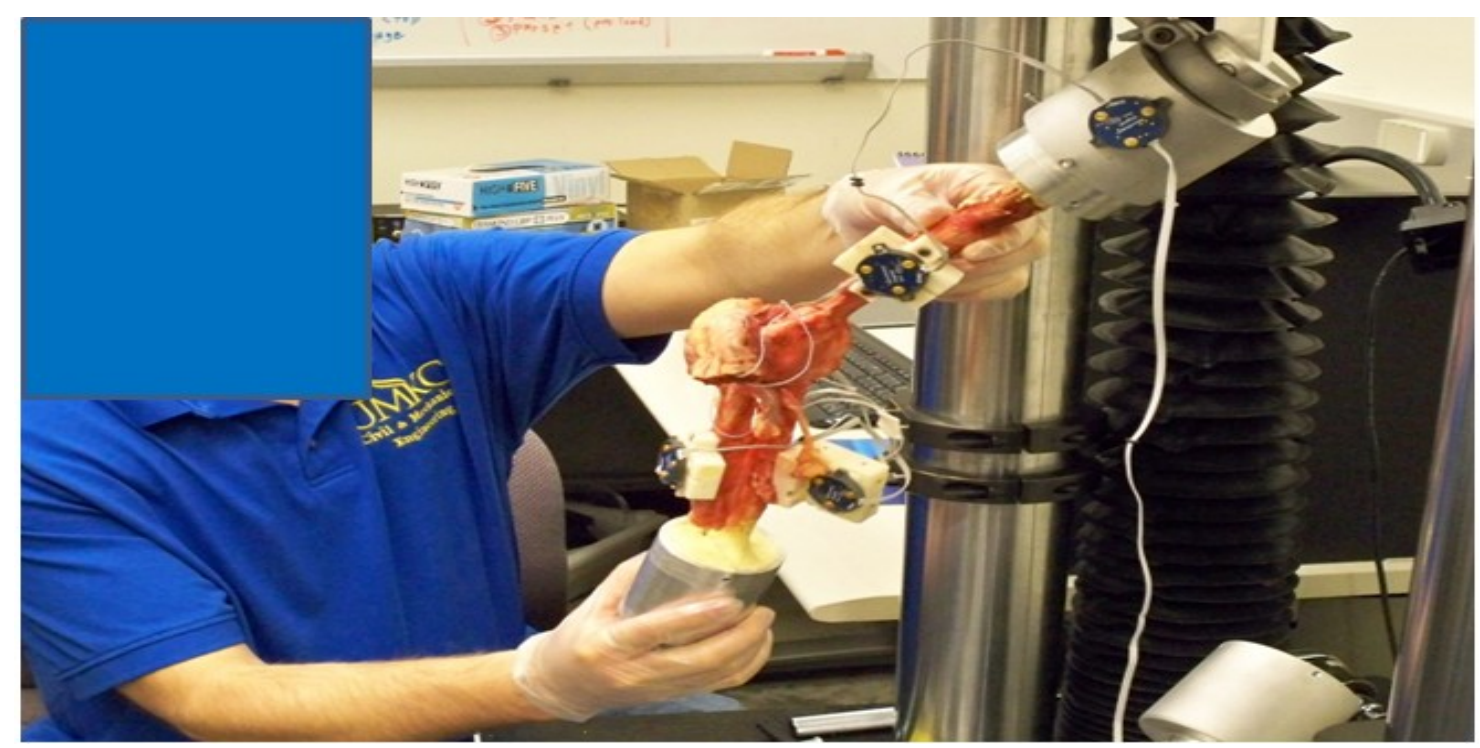

Figure 3.3. Laxity test to measure the zero load length.

Once the laxity test was completed, the arm was put back on the testing machine and the suture was pulled taut and secured to the load cell. The initial position and orientation of the cadaveric bone geometries relative to the mechanical tester were determined by recording multiple points on the localizers, along the bone surfaces, and on the load cell by using a probing tip of the Optotrak system. The mechanical tester was set at neutral position before 
starting a sinusoidal motion. A motion profile of $50 \mathrm{~mm}(25 \mathrm{~mm}$ downward and $25 \mathrm{~mm}$ upward from neutral position) vertical displacement was applied and recorded to the top ram. A 10 degree axial rotation ( \pm 5 degrees from equilibrium position) was also applied to the bottom ram with the same frequency of vertical displacement during testing. The approximate change of elbow flexion angle for each specimen resulting from the vertical motion of the top ram is shown in Table 3.2. For each experimental trial, the threedimensional coordinates obtained from each infrared marker as well as the associated triceps tendon forces from the load cell were recorded.

Table 3.2. Elbow flexion angle during movement.

\begin{tabular}{c|c|c|c}
\hline & $\begin{array}{c}\text { Initial flexion angle } \\
(\mathrm{deg})\end{array}$ & $\begin{array}{c}\text { Maximum flexion } \\
\text { angle(deg) }\end{array}$ & $\begin{array}{c}\text { Minimum flexion angle } \\
(\mathrm{deg})\end{array}$ \\
\hline Specimen\#1 & 75 & 82 & 65 \\
\hline Specimen\#2 & 91 & 96 & 86 \\
\hline Specimen\#3 & 76 & 83 & 68 \\
\hline
\end{tabular}

After all testing was completed; the elbow was dis-articulated and point clouds were collected along the articulating surfaces of the humerus, radius, and ulna along with ligament insertion and origins using the Optotrak digitizing probe. To minimize the error during point cloud collection, each segment was fixed by clamping it into a vice mounted to the work surface to keep them from moving. The main focus for the point clouds was to obtain the overall shape of the articular surfaces and to collect the insertion and origin sites for the ligaments and triceps tendon. Additional points were also taken on the surface of the sigmoid notch, capitellum, trochlea, coronoid, coronoid fossa, olecranon, olecranon fossa, radial head, 
radial neck, bicipital tuberosity, and on the diaphyses to aid in orienting the bone geometries during model generation.

\subsection{Multibody model formulations}

The program 3D Slicer (www.slicer.org) was used to generate three-dimensional bone geometries of the humerus, ulna, and radius from the two-dimensional Digital Imaging and Communications in Medicine (DICOM) files generated by Computed Tomography (CT) scan and Magnetic Resonance Imaging (MRI). An auto thresholding tool available in 3D slicer was used to isolate bone geometries from the CT scan images (Fig. 3.4) and a manual segmentation tool was used for MRI. For manual segmentation, a Wacom Cintiq 12wx Interactive Pen Display (Wacom Company, Ltd., Tokyo, Japan) tablet was used to create segment outline of geometries by hand. 


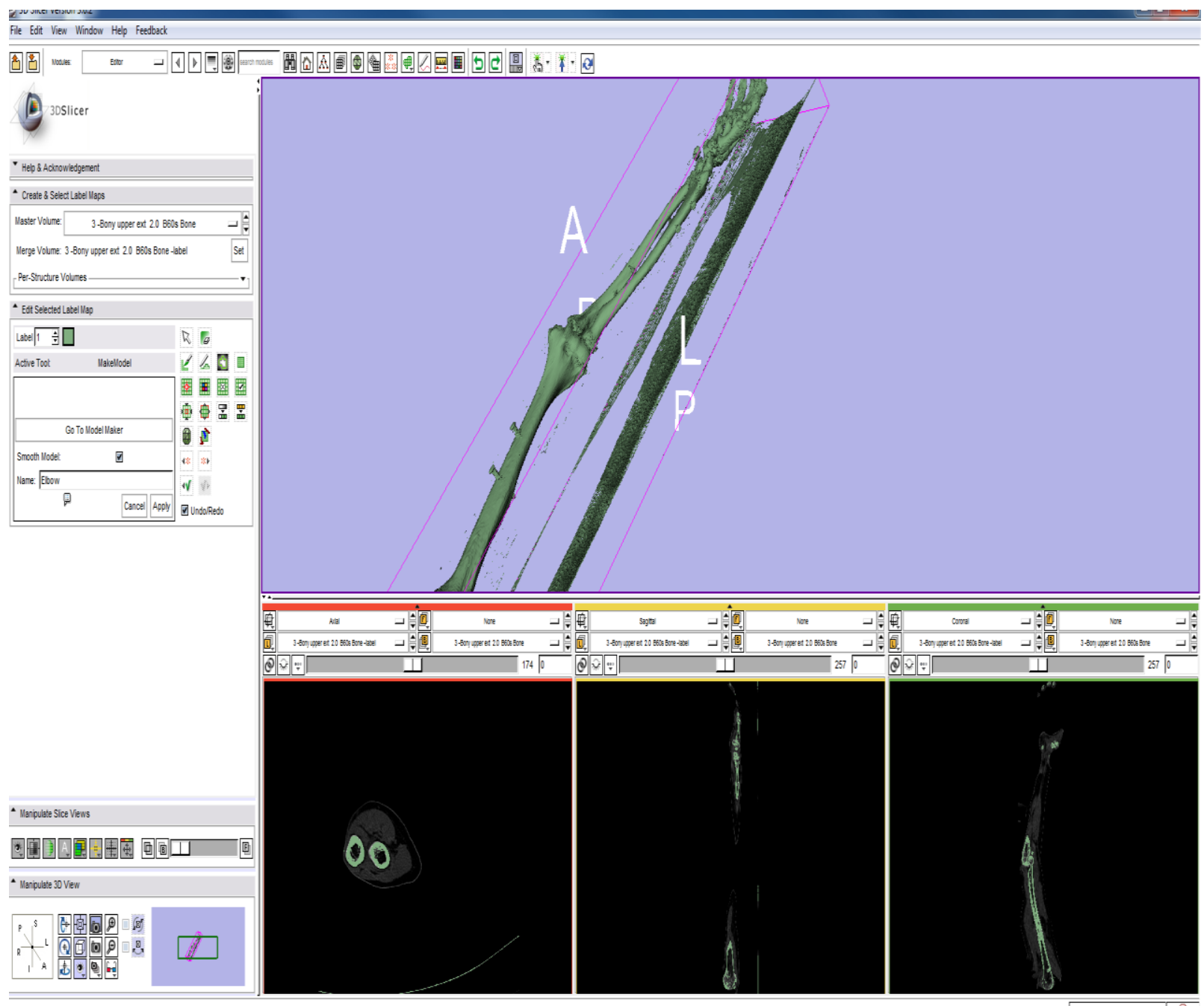

Figure 3.4. Auto threshold segmentation of $\mathrm{CT}$ images to isolate the bone geometries in 3D Slicer.

The geometries from 3D Slicer were imported into Geomagic Studio (Geomagic, Inc. Research Triangle Park, NC) as STereoLithography (STL) files for post-processing (Fig. 3.5). The processing included removing spikes from the geometry by setting a threshold to a maximum height relative to its surrounding points. A reduce noise command was also used to smooth serrated features produced by the geometry segmentation. The file size was reduced by decimating the geometries while maintaining the anatomical shape and volume. 
The cartilage geometries were extracted as solid bodies of uniform thickness from the articulating surfaces of the respective bones by using a feature available in Geomagic Studio.
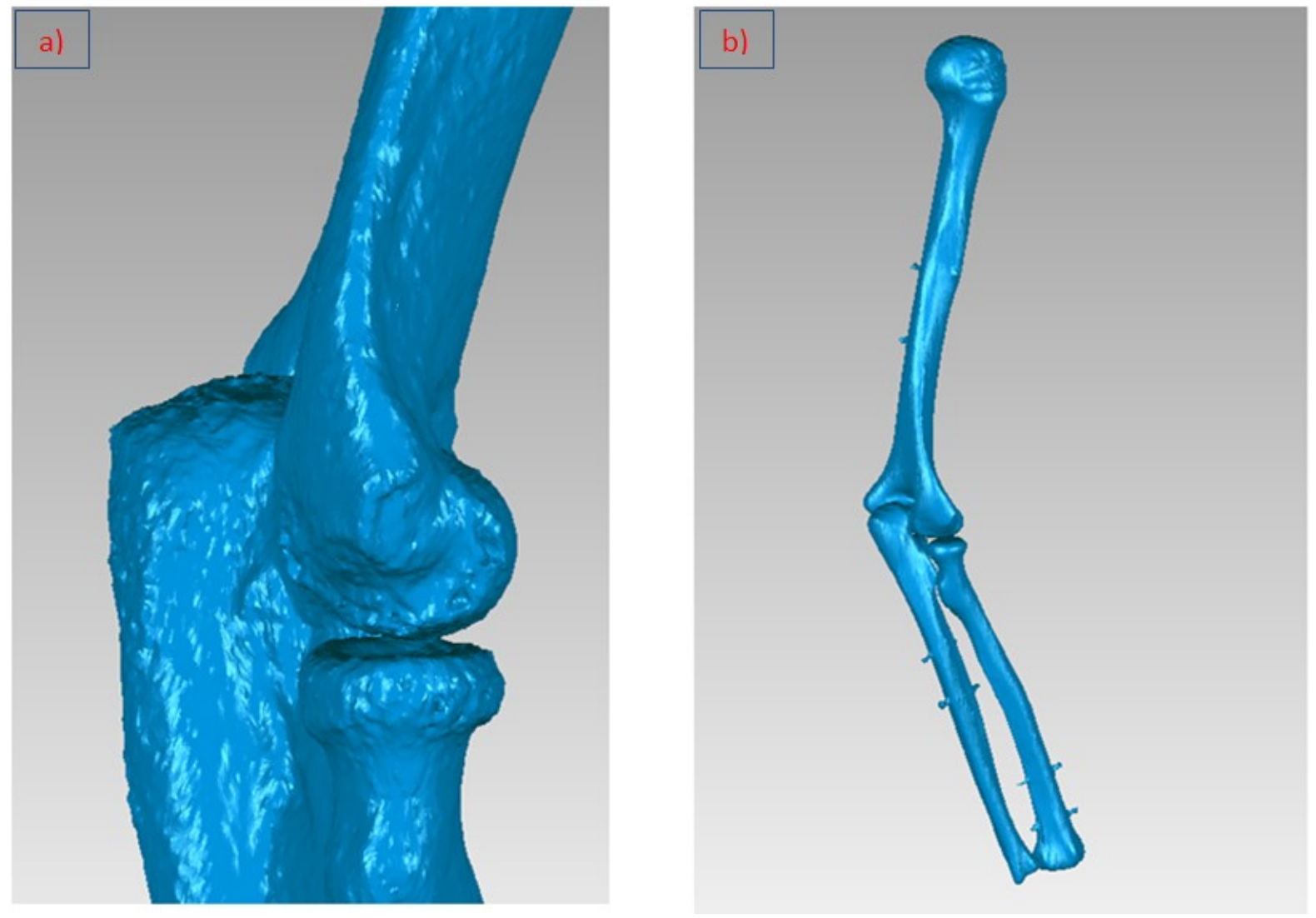

Figure 3.5. Three-dimensional bone geometries a) before and b) after post processing in Geomagic Studio.

A model was created in MD Adams (MSC Software Corporation, Santa Ana, CA) by importing the geometries of the bones, cartilages, top and bottom cylinder, and top ram mechanical tester. The geometries were then aligned by using the initial position and point clouds of each bone collected during the experimental testing (Fig. 3.6). 


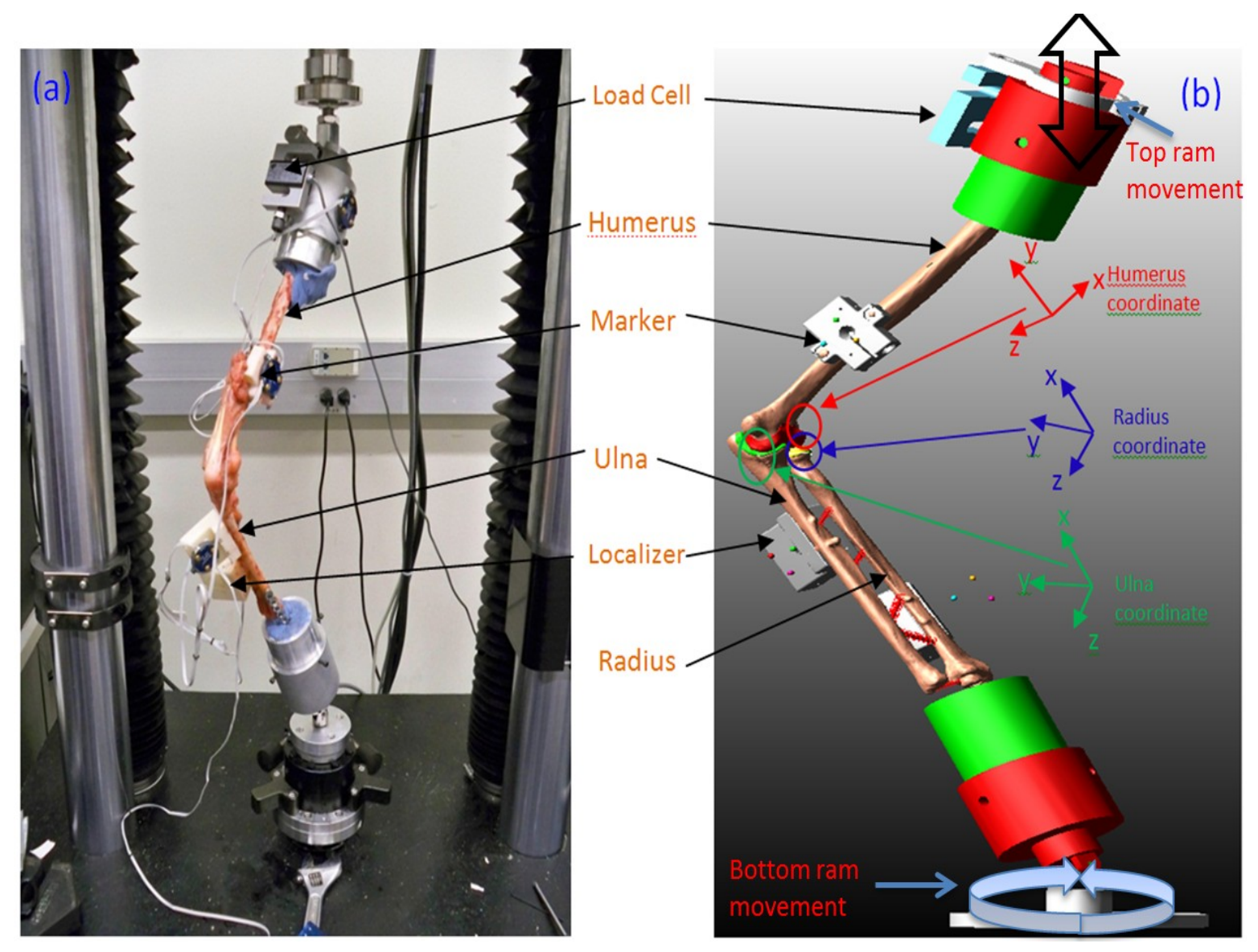

Figure 3.6. The experimental cadaver elbow setup in the mechanical tester. (b) Multibody model of the elbow in Adams. The approximate position of the humerus, ulna, and radius coordinate system are also indicated. The definition of the local elbow coordinate systems were obtained from Ferreira et al. (2011), and Morrey and Chao (1976).

The humerus and ulna geometries were attached by fixed joint with the top and bottom cylinders of the mechanical tester respectively. Similar to the experimental study, there was no constraint between the radius and testing machine. The top cylinder was attached by a hinge joint with the top ram and the bottom cylinder was attached by a universal joint with the bottom ram of the mechanical tester. The top ram of the mechanical tester was constrained by a translational joint with ground that allowed the model vertical 
movement. A density of $1600 \mathrm{~kg} / \mathrm{m}^{3}$ (Donahue et al., 2002) were defined for humerus, radius, and ulna bone and $1000 \mathrm{~kg} / \mathrm{m}^{3}$ for each articular cartilage (Zielinska \& Donahue, 2006).

The ligament and tendons were attached to the model according to the insertion and origin point cloud information collected during experimental testing. The point clouds were identified by an orthopaedic surgeon and were imported and added to their respective geometries. The ligaments were divided into different bundles according to their structure and function. The model included three bundles for the lateral ulnar collateral ligament (LUCL), three bundles for the radial collateral ligament (RCL) (Spratley \& Wayne, 2011), three bundles for the medial collateral ligament (MCL) anterior part, three bundles for MCL posterior part, and two bundles for the annular ligament (Fisk \& Wayne, 2009).

The ligaments and tendon were modeled as non-linear springs using a piecewise function describing the force-length relationship including the non-linear "toe" region to describe the characteristics of human ligaments. The force-length relationship is described by equations (3-1) and (3-2) (Blankevoort, Kuiper, Huiskes, \& Grootenboer, 1991; Wismans et al., 1980).

$$
\begin{aligned}
& f=\left\{\begin{array}{cllll}
\frac{1}{4} k \varepsilon^{2} / \varepsilon_{l} & 0 \leq \varepsilon \leq 2 \varepsilon_{l} & & & \\
k\left(\varepsilon-\varepsilon_{l}\right) & \varepsilon>2 \varepsilon_{l} & \ldots & \ldots & \ldots(3-1) \\
0 & \varepsilon<0 & & &
\end{array}\right. \\
& \varepsilon=\left(\frac{l-l_{0}}{l_{0}}\right) \ldots \quad \ldots \quad \ldots(3-2)
\end{aligned}
$$

Here, $\mathrm{k}$ is the stiffness parameter, $\varepsilon_{l}$ is a spring parameter assumed to be 0.03 ( $\mathrm{Li}$ et al., 1999), and $\varepsilon$ is the ligament engineering strain (the ratio of range of motion divided by initial length (equation (3-2)). The stiffness parameter $(\mathrm{k})$ is defined in units of force $(\mathrm{N})$ and is derived from the stiffness coefficient $(\mathrm{N} / \mathrm{mm})$ by multiplying it by the ligament bundle zero 
load length $(\mathrm{mm})$. The zero strain regions resemble the ligament behavior when the ligament length is less than the zero load length. The toe region corresponds to the parabolic transition between the zero strain and linear regions that simulate the nonlinearity and crimping effect of the ligament (Fig. 3.7). The linear region increases at a constant rate with the application of forces to the ligament.

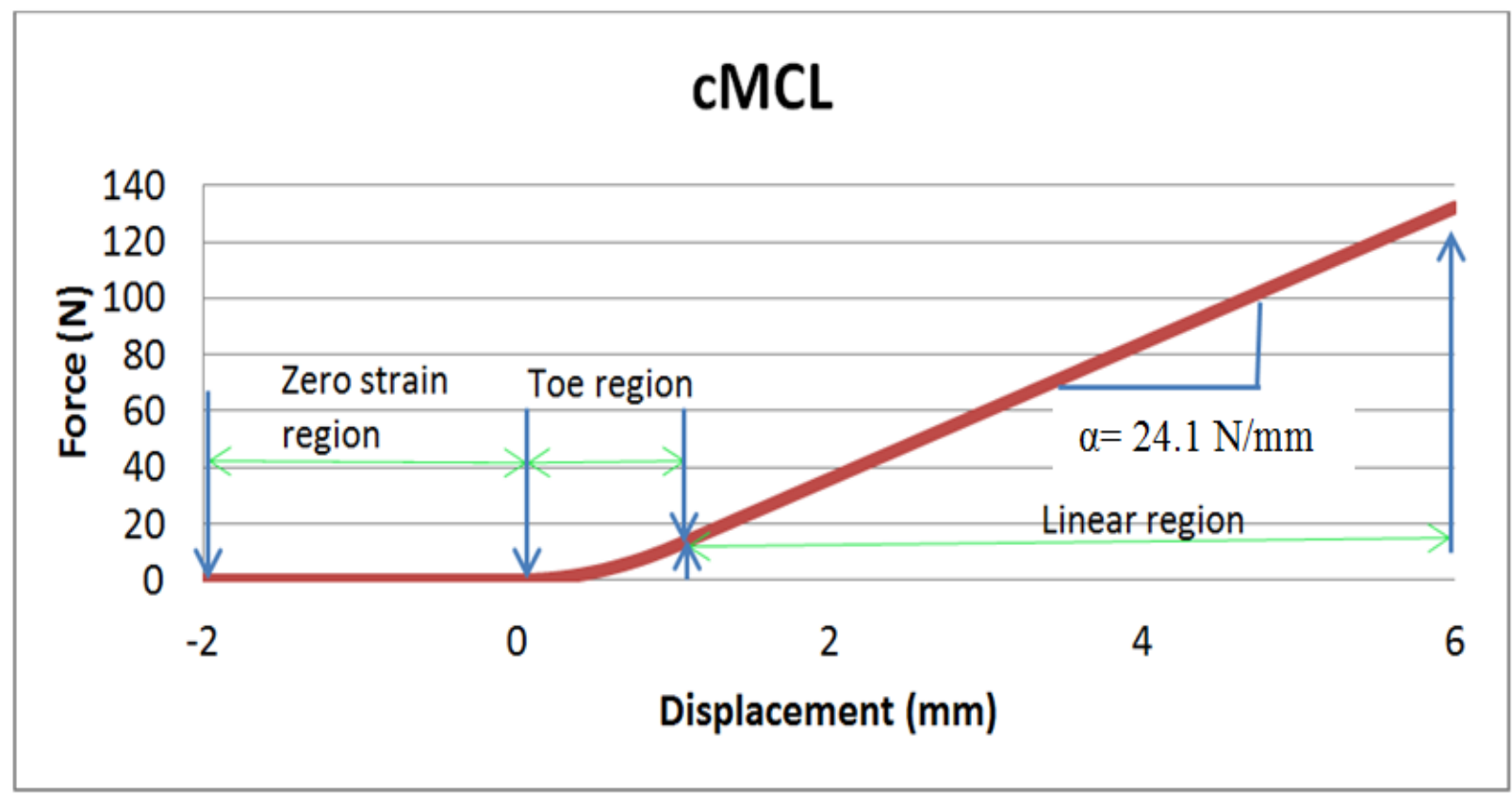

Figure 3.7. The force-displacement relationship for the central bundle of the medial collateral ligament (cMCL) anterior part. The measured zero-load length of the cMCL was $17.2 \mathrm{~mm}$ and the stiffness coefficient in the linear region was $24.1 \mathrm{~N} / \mathrm{mm}$.

A custom subroutine was written in ADAMS to implement equation (3-1) in the model. Input to the subroutine included the ligament insertion and origin point positions, the ligament stiffness parameter, damping coefficient, spring parameter, and the measured zeroload length. The stiffness coefficient $(\alpha)$ for each ligament bundle shown in Table 3.3 came 
from published literature (Fisk \& Wayne, 2009; Regan, Korinek, Morrey, \& An, 1991; Spratley \& Wayne, 2011)

Table 3.3. Ligament modeling parameters. (Fisk \& Wayne, 2009; Regan et al., 1991; Spratley \& Wayne, 2011)

\begin{tabular}{|c|c|c|c|c|c|}
\hline \multirow{2}{*}{$\begin{array}{l}\text { Ligament } \\
\text { Bundle }\end{array}$} & \multirow[b]{2}{*}{ Description } & \multirow{2}{*}{$\begin{array}{l}\text { Stiffness } \\
\text { coefficient } \\
(\mathrm{N} / \mathrm{mm})\end{array}$} & \multicolumn{3}{|c|}{ Zero-load length (mm) } \\
\hline & & & $\begin{array}{c}\text { Specimen } \\
1\end{array}$ & $\begin{array}{c}\text { Specimen } \\
2\end{array}$ & $\begin{array}{c}\text { Specimer } \\
3\end{array}$ \\
\hline aLUCL & $\begin{array}{l}\text { Lateral Ulnar Collateral } \\
\text { Ligament, anterior bundle }\end{array}$ & 19.0 & 28.3 & 36.4 & 34.2 \\
\hline cLUCL & $\begin{array}{l}\text { Lateral Ulnar Collateral } \\
\text { Ligament, central bundle }\end{array}$ & 19.0 & 29.8 & 37.3 & 36.7 \\
\hline pLUCL & $\begin{array}{l}\text { Lateral Ulnar Collateral } \\
\text { Ligament, posterior bundle }\end{array}$ & 19.0 & 34.3 & 38.5 & 38.8 \\
\hline aMCL & $\begin{array}{l}\text { Medial Collateral Ligament, } \\
\text { Anterior part, ant. bundle }\end{array}$ & 24.1 & 18.3 & 25.1 & 18.3 \\
\hline cMCL & $\begin{array}{l}\text { Medial Collateral Ligament, } \\
\text { Anterior part, cent. bundle }\end{array}$ & 24.1 & 19.2 & 24.6 & 18.0 \\
\hline pMCL & $\begin{array}{l}\text { Medial Collateral Ligament, } \\
\text { Anterior part, post. bundle }\end{array}$ & 24.1 & 19.8 & 23.6 & 17.3 \\
\hline PBAB & $\begin{array}{l}\text { MCL, Posterior part, ant. } \\
\text { bundle }\end{array}$ & 17.4 & 15.5 & 20.2 & 14.9 \\
\hline PBCB & $\begin{array}{l}\text { MCL, Posterior part, cent. } \\
\text { bundle }\end{array}$ & 17.4 & 15.3 & 19.3 & 13.5 \\
\hline PBPB & $\begin{array}{l}\text { MCL, Posterior part, post. } \\
\text { bundle }\end{array}$ & 17.4 & 15.6 & 22.9 & 15.7 \\
\hline $\mathrm{aRCL}$ & $\begin{array}{l}\text { Radial Collateral Ligament, } \\
\text { anterior bundle }\end{array}$ & 15.5 & 18.4 & 22.5 & 15.2 \\
\hline $\mathrm{cRCL}$ & $\begin{array}{l}\text { Radial Collateral Ligament, } \\
\text { central bundle }\end{array}$ & 15.5 & 17.6 & 21.7 & 14.5 \\
\hline $\mathrm{pRCL}$ & $\begin{array}{l}\text { Radial Collateral Ligament, } \\
\text { posterior bundle }\end{array}$ & 15.5 & 18.3 & 22.6 & 14.2 \\
\hline ALAB & $\begin{array}{l}\text { Annular Ligament, proximal } \\
\text { bundle }\end{array}$ & 28.5 & - & - & - \\
\hline ALPB & $\begin{array}{l}\text { Annular Ligament, distal } \\
\text { bundle }\end{array}$ & 28.5 & - & - & - \\
\hline
\end{tabular}

A damping coefficient of $0.5 \mathrm{Ns} / \mathrm{mm}$ was included in each spring element to remove the possibility of high frequency vibration during simulation (Guess, 2012). The triceps 
tendon was also modeled as a single bundle nonlinear spring damper element using a stiffness parameter $(0.2 \mathrm{~N} / \mathrm{mm})$ obtained from Tate (2012). The distal radioulnar joint ligament (For specimen 2 and 3) and interosseous membrane were modeled as five bundles and two bundles of linear spring elements respectively. The stiffness coefficient (Table 3.4) as well as attachment points (Fig. 3.8) of interosseous membrane in three-dimensional space were obtained from the literature Fisk (2007); (Peck et al., 2000; Schuind et al., 1991).

Table 3.4. Stiffness parameters for interosseous membrane. (Fisk, 2007; Peck et al., 2000; Schuind et al., 1991)

\begin{tabular}{c|c|c}
\hline Tissue part & Bundle name & Stiffness (N/mm) \\
\hline \multirow{4}{*}{$\begin{array}{c}\text { Interosseous } \\
\text { membrane }\end{array}$} & Accessory part, Distal bundle & 18.9 \\
\cline { 2 - 3 } & Accessory part, proximal bundle & 18.9 \\
\cline { 2 - 3 } & Central part, Distal bundle & 65.0 \\
\cline { 2 - 3 } & Central part, proximal bundle & 65.0 \\
\hline \multirow{2}{*}{$\begin{array}{c}\text { Distal } \\
\text { radioulnar } \\
\text { joint ligaments }\end{array}$} & Distal oblique bundle & 65.0 \\
\cline { 2 - 3 } & Dorsal bundle & 13.2 \\
\hline
\end{tabular}

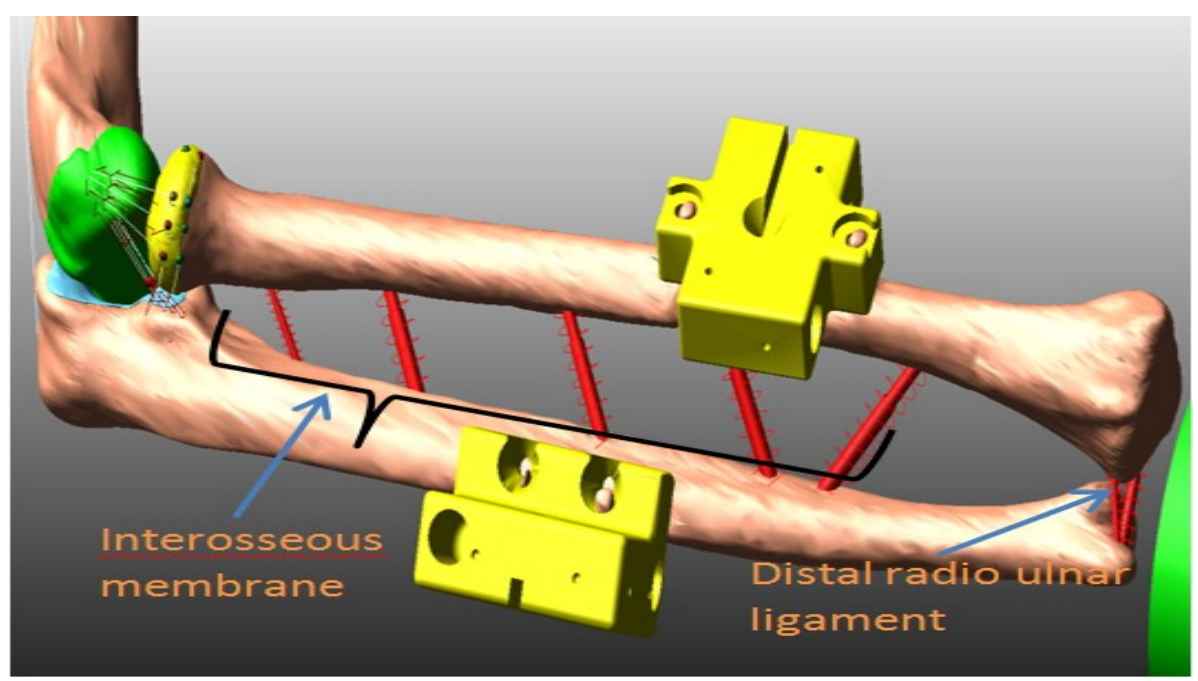

Figure 3.8. Interosseous membrane and distal radioulnar ligaments in the model. Right limb shown. 
Because of wrapping around the bone of the annular and lateral ulnar collateral ligament (LUCL), it would be ineffective to define these ligaments as direct ligament line of action. To simulate wrapping for these ligaments, each ligament bundle was divided into multiple elements attached in series according to their path structure (Fig. 3.9). For the LUCL, ellipsoids with a diameter equal to ligament thickness were inserted into the ligament. Deformable contact constraints using equation (3-3) were then defined between the ellipsoids and radius cartilage allowing the ellipsoids to slide over the radial head. For the annular ligament wrapping, small spheres were embedded in the ligament and one line arc for each ligament bundle was placed along the perimeter of the radius head. A point-curve constraint was then defined between the spheres and line arcs, allowing the spheres to move along the path of the curves. Therefore, the radius could rotate inside the annular ligament during forearm pronation-supination, similar to its physiological constraint. To restrain the ligament elements from crossing each other during simulation, parallel elements of the LUCL were connected with spring elements (Fig. 3.9). 


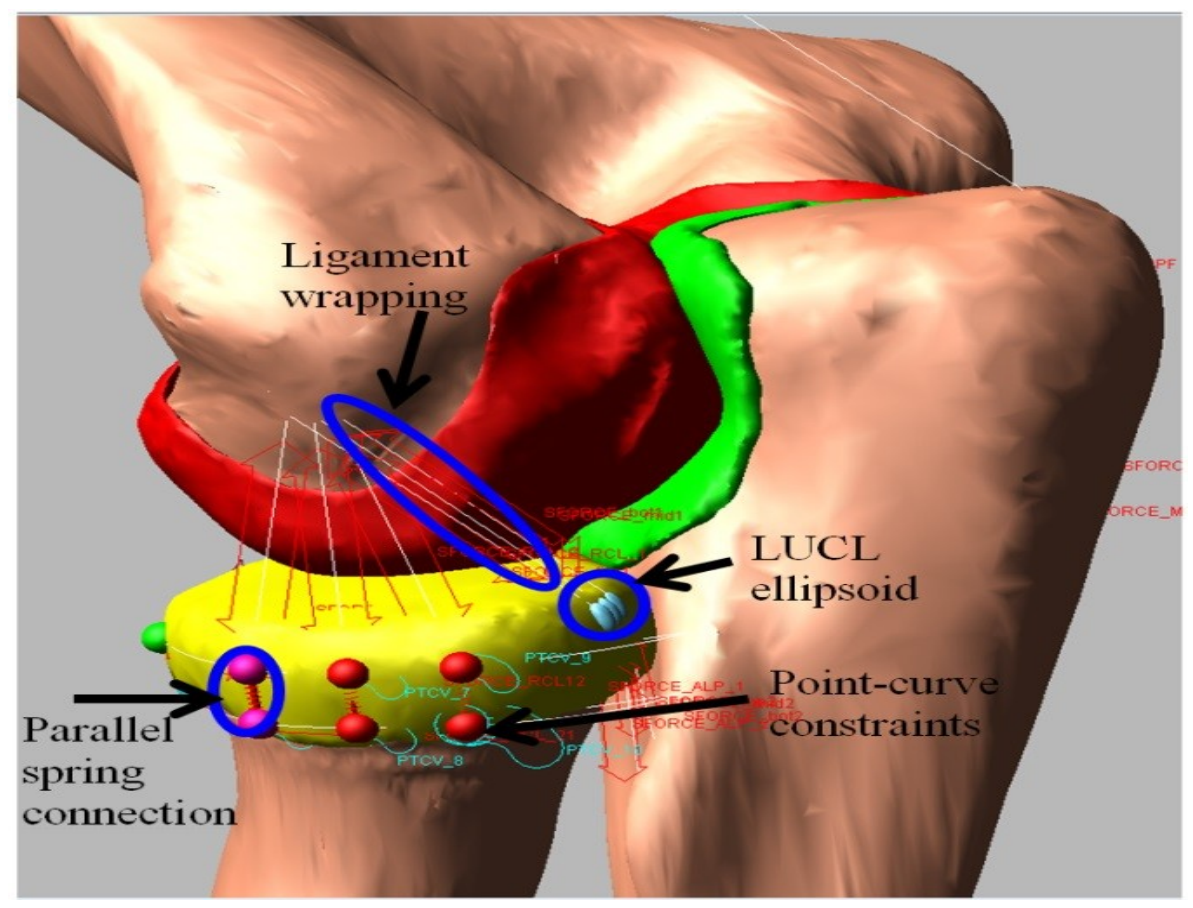

Figure 3.9. Wrapping of the LUCL and annular ligament around the bone. Also shown is the point-curve constraints and parallel connection between two spheres of the LUCL and annular ligaments.

Deformable contact constraints with no friction were defined between the articulating geometries by using a modified Hertzian contact law defined in ADAMS as:

$$
F_{c}=k_{c} \delta^{n}+B_{c}(\delta) \dot{\delta} \ldots \ldots(3-3)
$$

where $F_{c}$ is the contact force, $k_{c}$ is the contact stiffness, $\delta$ is the interpenetration of the geometries, $n$ is the nonlinear power exponent, $\dot{\delta}$ is the velocity of interpenetration, and $B_{c}(\delta)$ is a damping coefficient. To prevent discontinuities in the solution for when the rigid bodies first come in contact, the damping co-efficient was a function of interpenetration (Hunt \& Crossley, 1975). 
The contacts were defined by using equation (3-3) between the humerus and radius, the humerus and ulna, the radius and ulna, the humerus cartilage and radius cartilage, the humerus cartilage and ulna cartilage, and the ulna cartilage and radius cartilage. The geometries were converted into the Parasolid geometric modeling kernel before defining the contacts. Parasolids are three dimensional solids with continuous representation. If contact occurs between geometries, the ADAMS contact model computes the contact location, the contact normal force, and the contact penetration depth $(\delta)$. The contact parameters used for this study are shown in Table 3.5.

Table 3.5. Contact parameters information

\begin{tabular}{c|c}
\hline Parameters & Values \\
\hline Contact type & Impact (Deformable) \\
\hline Friction & No \\
\hline Stiffness $\left(k_{c}\right)$ & $\begin{array}{c}\text { N/mm (bone to bone contact), } 200 \mathrm{~N} / \mathrm{mm} \\
\text { (cartilage to cartilage contact) }\end{array}$ \\
\hline Interpenetration of geometries $(\delta)$ & $0.1 \mathrm{~mm}$ \\
\hline Exponent $(\mathrm{n})$ & 1.5 \\
\hline Damping coefficient $\left(\mathrm{B}_{\mathrm{c}}(\delta)\right)$ & $20 \mathrm{Ns} / \mathrm{mm}$ \\
\hline
\end{tabular}

Local coordinate systems for each bone segment were created as described by Ferreira et al. (2011) and Morrey and Chao (1976) to measure the segment motion of the model (Fig. 3.10). The origin for the humeral coordinate system was taken at the center of the capitellum. The capitellum was fitted with sphere a least squares sense to find its center. The positive $\mathrm{Z}$ axis was defined from the center of the capitellum to direct medially to the center of the trochlear sulcus. The $\mathrm{Z}$ axis formed the flexion-extension axis of the elbow joint. The positive $\mathrm{X}$ axis was defined from the center of the capitellum to proximally direct to the center of the humeral head. The $+\mathrm{Y}$ axis was made from the vector cross product of the 
$+\mathrm{Z}$ and $+\mathrm{X}$ axes and directed anteriorly for a right arm and posteriorly for a left arm. The $\mathrm{X}$, $\mathrm{Y}$ and $\mathrm{Z}$ axes correspond approximately to the superior-inferior (S-I), anterior-posterior (A$\mathrm{P})$, and medial-lateral (M-L) direction respectively.

The origin of ulnar coordinate system was placed to the center of the greater sigmoid notch obtained from fitting with a circle in a least squares sense. The $+Z$ axis was defined from the center of the circle and directed medially to the normal vector of the plane, forming the ulnar flexion axis. The $+\mathrm{X}$ axis was defined by a line from the distal ulnar styloid to the center of the greater sigmoid notch. The $+Y$ axis was made by the vector cross product of the $+\mathrm{Z}$ and $+\mathrm{X}$ axis that directed anteriorly for a right arm and posteriorly for a left arm.

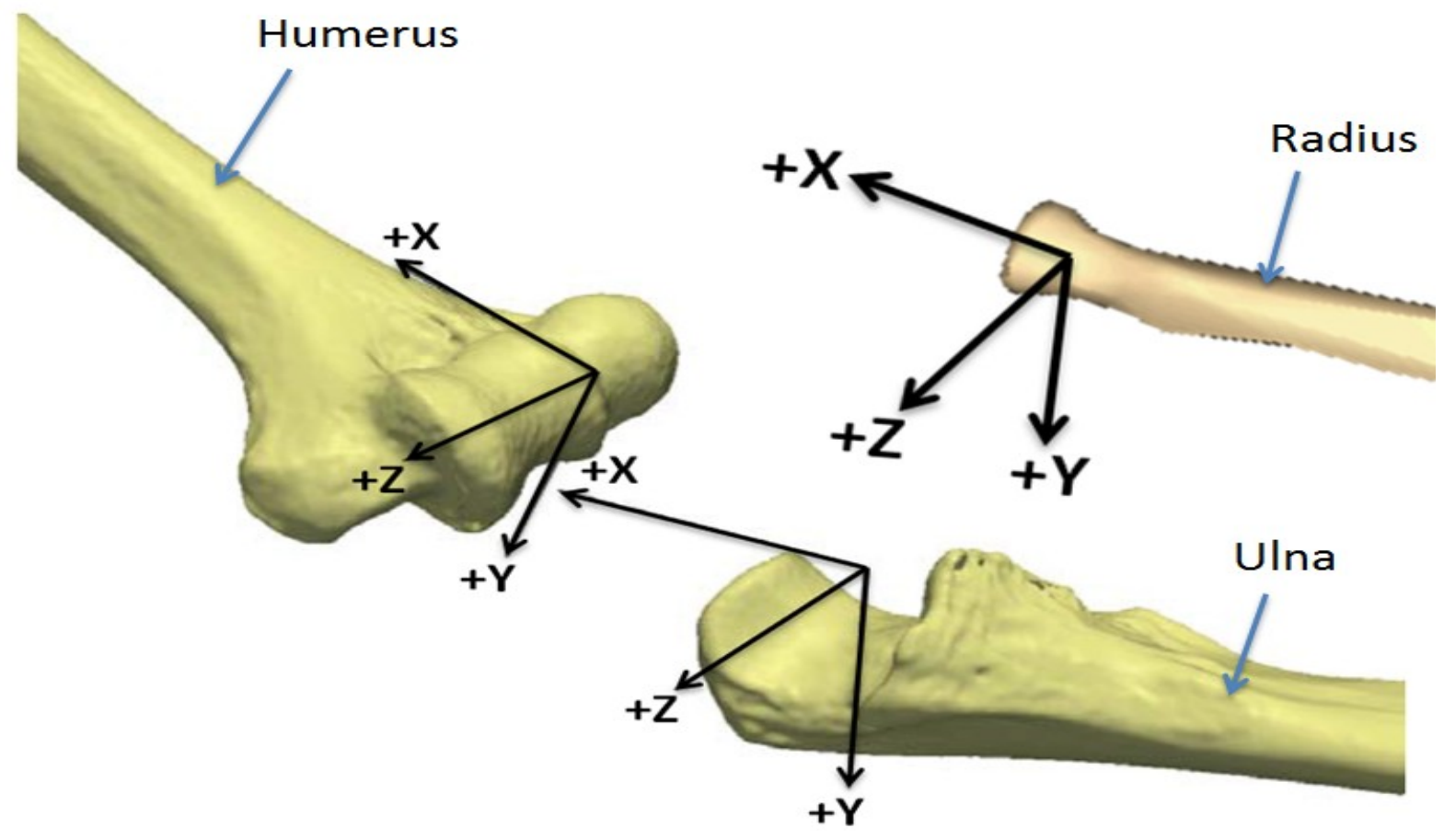

Figure 3.10. Approximate position and orientation of the elbow joint coordinate system. Source: Ferreira (2011). 
The origin of the radius coordinate system was located at the center of the radial head that was obtained from sphere fit in a least squares sense. The $+Z$ axis was defined from the center of the radial head and oriented similar to the humeral $+Z$ axis. The $+X$ axis was defined by a line from the center of the distal radius diaphysis to the center of the radial head. The $+\mathrm{Y}$ axis was made by the vector cross product of $+\mathrm{Z}$ and $+\mathrm{X}$ axis that directed anteriorly for a right arm and posteriorly for a left arm. The translations of the radius and ulna were computed from the origin of their respective local coordinate system relative to the humerus local coordinate system and were presented in humerus coordinates. The rotations were represented in a 123 Euler angle sequence (Body 1, 2 and 3 angles) which correspond to internal-external rotation (I-E), adduction-abduction (AD-AB duction), and flexion-extension (F-E) of the joint motion.

The model was then subjected to the same $50 \mathrm{~mm}$ upwards-downwards motion profile on the top ram and $10^{\circ}$ axial rotations on the bottom ram as the cadaver experimental testing. Finally for each simulation, the kinematics of each segment along with the forces on the triceps tendon was predicted. Another simulation was conducted to observe the kinematic difference for a linear-ligament model (the model that did not include ligament 'toe' region). To implement this in the model, the ligament spring parameter $\left(\varepsilon_{l}\right)$ were assumed to be very small $\left(3 \times 10^{-11}\right)$. An additional simulation was run to observe the kinematic difference for a non-wrapping model (the model that did not include ligament wrapping).

The RMS error between the experimental and predicted kinematics were calculated by using equation 3.4 for all simulations in every ligament condition. The RMS error was also measured between experimental and predicted triceps tendon forces. 


$$
\mathbf{R M S}=\sqrt{\frac{\sum\left(\mathbf{x}_{\mathbf{k}}-\mathbf{x}_{\mathbf{s}}\right)^{2}}{\mathbf{n}}} \ldots \ldots \ldots(3-4)
$$

Where $\mathrm{x}_{\mathrm{k}}$ is the single component position or orientation for experimental data, $\mathrm{x}_{\mathrm{s}}$ is the single component position or orientation for predicted data, and $\mathrm{n}$ is the total number of data points

Analysis of variance (ANOVA) was conducted to examine the effect of ligament wrapping and ligament nonlinearity on model outputs. Kinematics RMS error against the experimental data from the non-linear-wrapping model (the model that included both nonlinear ligament and ligament wrapping) was taken as one sample (Table 4.1) for ANOVA calculation. Similarly, the kinematic RMS error against experimental data from nonwrapping model was taken as second set of sample data. IBM SPSS (IBM Corporation, Armonk, NY, USA) statistics software was used to calculate the ANOVA from those two sets of data, and the F-ratios (the ratio of sample variances) were used to evaluate the significance of changing factors. A similar approach was followed for ANOVA calculation to compare the RMS error between non-linear-wrapping and the linear model. The p-value was then used to measure the statistical significance and percentage confidence on the results. If the p-value was less than 0.05 , then the change was statistically significant for the result. Conversely, if the p-value was greater than 0.05 , then the factor change was not statistically significant. 


\section{CHAPTER 4}

\section{RESULT}

Model predicted displacement and rotations of the ulna and radius coordinate relative to the humerus coordinate were compared to experimental data for specimen 1 (Figs. 4.14.12). The same conditions were followed for all other specimens (Appendix A). The predicted triceps tendon forces were also compared to the force obtained from the load cell (Fig. 4.13). The RMS error (Table 4.1) between the experimental and predicted kinematics and triceps tendon force were calculated to quantify how well the model followed the experiment. 


\subsection{RMS error}

Table 4.1. RMS error between predicted and measured kinematics and triceps tendon forces. Errors are shown for each ligament condition. The top values for each axis are shown in bold.

\begin{tabular}{|c|c|c|c|c|c|c|c|c|c|c|c|c|c|}
\hline & & \multicolumn{6}{|c|}{ Ulna Coordinate system } & \multicolumn{6}{|c|}{ Radius Coordinate system } \\
\hline & & \multicolumn{3}{|c|}{$\begin{array}{l}\text { Translation } \\
(\mathrm{mm})\end{array}$} & \multicolumn{3}{|c|}{$\begin{array}{l}\text { Rotation } \\
\text { (deg) }\end{array}$} & \multicolumn{3}{|c|}{$\begin{array}{c}\text { Translation } \\
(\mathrm{mm})\end{array}$} & \multicolumn{3}{|c|}{$\begin{array}{l}\text { Rotation } \\
\text { (deg) }\end{array}$} \\
\hline & & S-I & A-P & M-L & $\begin{array}{c}\text { Body } \\
1\end{array}$ & $\begin{array}{c}\text { Body } \\
2\end{array}$ & $\begin{array}{c}\text { Body } \\
3\end{array}$ & S-I & A-P & M-L & $\begin{array}{c}\text { Body } \\
1\end{array}$ & $\begin{array}{l}\text { Body } \\
2\end{array}$ & $\begin{array}{c}\text { Body } \\
3\end{array}$ \\
\hline \multirow{3}{*}{ 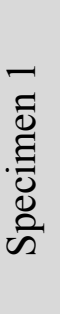 } & $\begin{array}{c}\text { Non- } \\
\text { linear } \\
\text {-wrapping }\end{array}$ & 1.2 & 1.3 & 1.8 & 0.2 & 0.1 & 0.2 & 0.7 & 1.0 & 1.9 & 2.4 & 2.7 & 0.6 \\
\hline & $\begin{array}{c}\text { Linear } \\
\text {-ligament }\end{array}$ & 0.8 & 1.3 & 1.8 & 0.6 & 0.1 & 0.2 & 1.0 & 1.3 & 2.6 & 2.7 & 4.2 & 0.7 \\
\hline & $\begin{array}{c}\text { Non- } \\
\text { wrapping }\end{array}$ & 1.2 & 1.2 & 1.9 & 0.3 & 0.1 & 0.3 & 0.8 & 1.1 & 2.5 & 2.6 & 4.4 & 0.6 \\
\hline \multirow{3}{*}{ 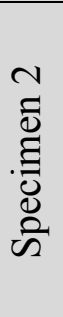 } & $\begin{array}{c}\text { Non- } \\
\text { linear- } \\
\text { wrapping }\end{array}$ & 1.6 & 1.5 & 4.6 & 0.6 & 0.2 & 0.1 & 1.4 & 1.6 & 4.5 & 1.0 & 1.9 & 0.3 \\
\hline & $\begin{array}{c}\text { Linear- } \\
\text { ligament }\end{array}$ & 1.8 & 1.5 & 4.6 & 0.6 & 0.2 & 0.2 & 1.1 & 1.8 & 4.9 & 1.0 & 1.9 & 0.1 \\
\hline & $\begin{array}{c}\text { Non- } \\
\text { wrapping }\end{array}$ & 1.6 & 1.7 & 4.6 & 0.6 & 0.2 & 0.1 & 1.2 & 1.8 & 4.6 & 0.6 & 1.3 & 0.2 \\
\hline \multirow{3}{*}{ 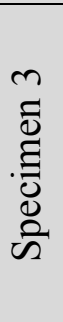 } & $\begin{array}{c}\text { Non- } \\
\text { linear- } \\
\text { wrapping }\end{array}$ & 1.4 & 1.9 & 3.0 & 0.5 & 0.6 & 0.6 & 1.4 & 2.1 & 3.1 & 3.3 & 5.3 & 0.3 \\
\hline & $\begin{array}{c}\text { Linear- } \\
\text { ligament }\end{array}$ & 1.1 & 1.9 & 3.0 & 0.4 & 0.5 & 0.5 & 1.3 & 2.1 & 3.4 & 3.3 & 5.3 & 0.5 \\
\hline & $\begin{array}{c}\text { Non- } \\
\text { wrapping }\end{array}$ & 0.7 & 2.0 & 3.5 & 0.5 & 0.4 & 0.4 & 1.2 & 1.9 & 2.8 & 1.5 & 2.4 & 0.5 \\
\hline & $\begin{array}{l}\text { Triceps } \\
\text { Tendon }\end{array}$ & \multicolumn{4}{|c|}{$\begin{array}{c}\text { Specimen } 1 \\
3.9\end{array}$} & \multicolumn{4}{|c|}{$\begin{array}{c}\text { Specimen } 2 \\
2.6\end{array}$} & \multicolumn{4}{|c|}{$\begin{array}{c}\text { Specimen } 3 \\
7.5\end{array}$} \\
\hline
\end{tabular}

Table 4.1 gives the RMS error between the measured and predicted kinematics and triceps tendon forces. The maximum value for each axis is shown in bold. As shown in the table, the peak translational error occurs along the medial-lateral direction for radius coordinate system for specimen 2 and the value reaches $4.9 \mathrm{~mm}$. The maximum rotational 
error of $5.3^{\circ}$ occurs for specimen 3 for body 2 rotation of the radius coordinate system. The largest translational error for the ulna happens along the medial-lateral direction for specimen 3 and the value reach $4.6 \mathrm{~mm}$. The largest rotation error for the ulna occurs in the body 1 rotation of the 123 sequence (internal/external rotation) for specimen $2\left(0.6^{\circ}\right)$. The greatest RMS error is $7.5 \mathrm{~N}$ for the triceps tendon force in specimen 3 .

\subsection{Ulna kinematics}

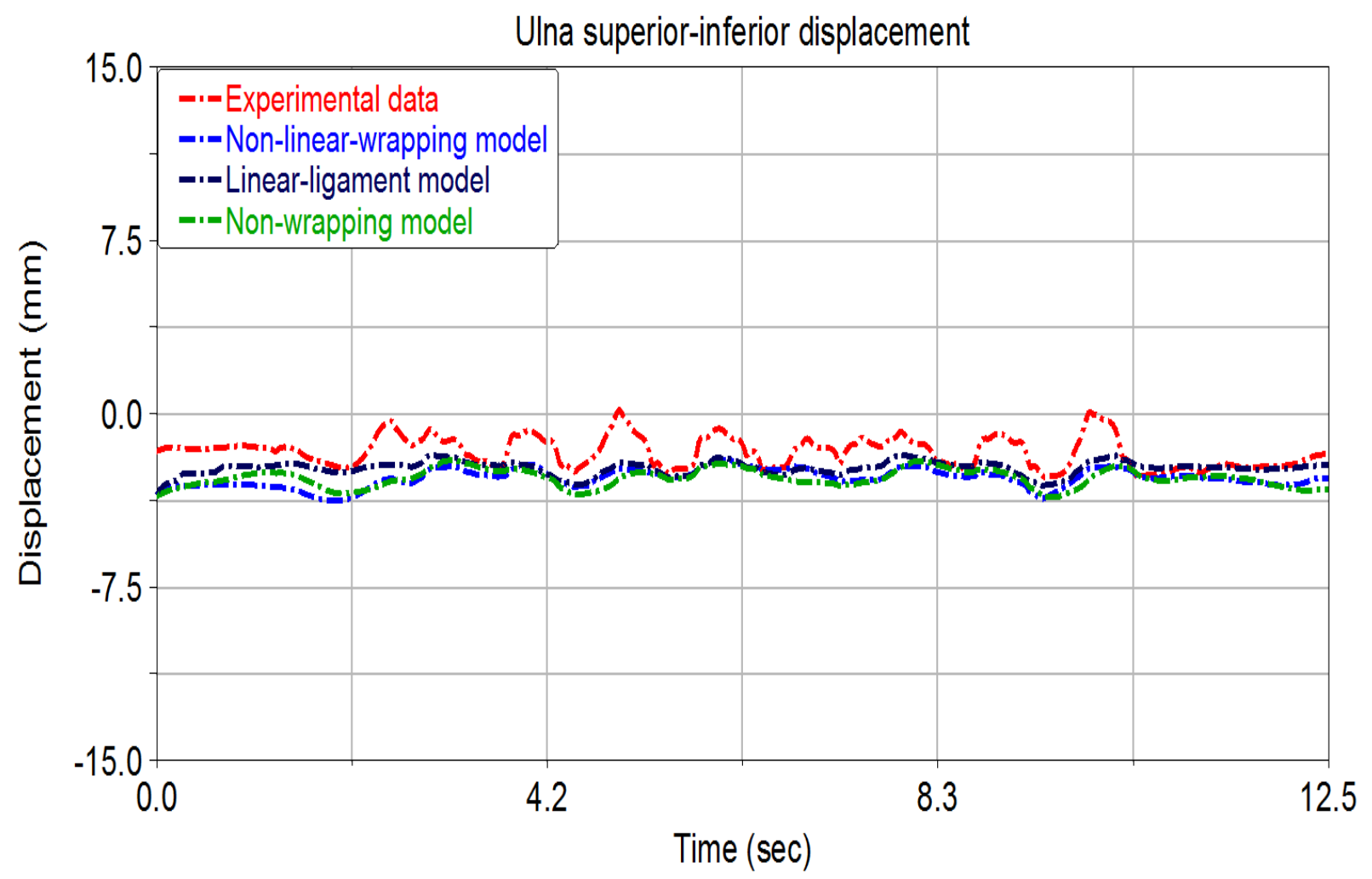

Figure 4.1. Superior-inferior (S-I) displacement of the ulna coordinate system relative to the humerus coordinates for specimen 1 . 


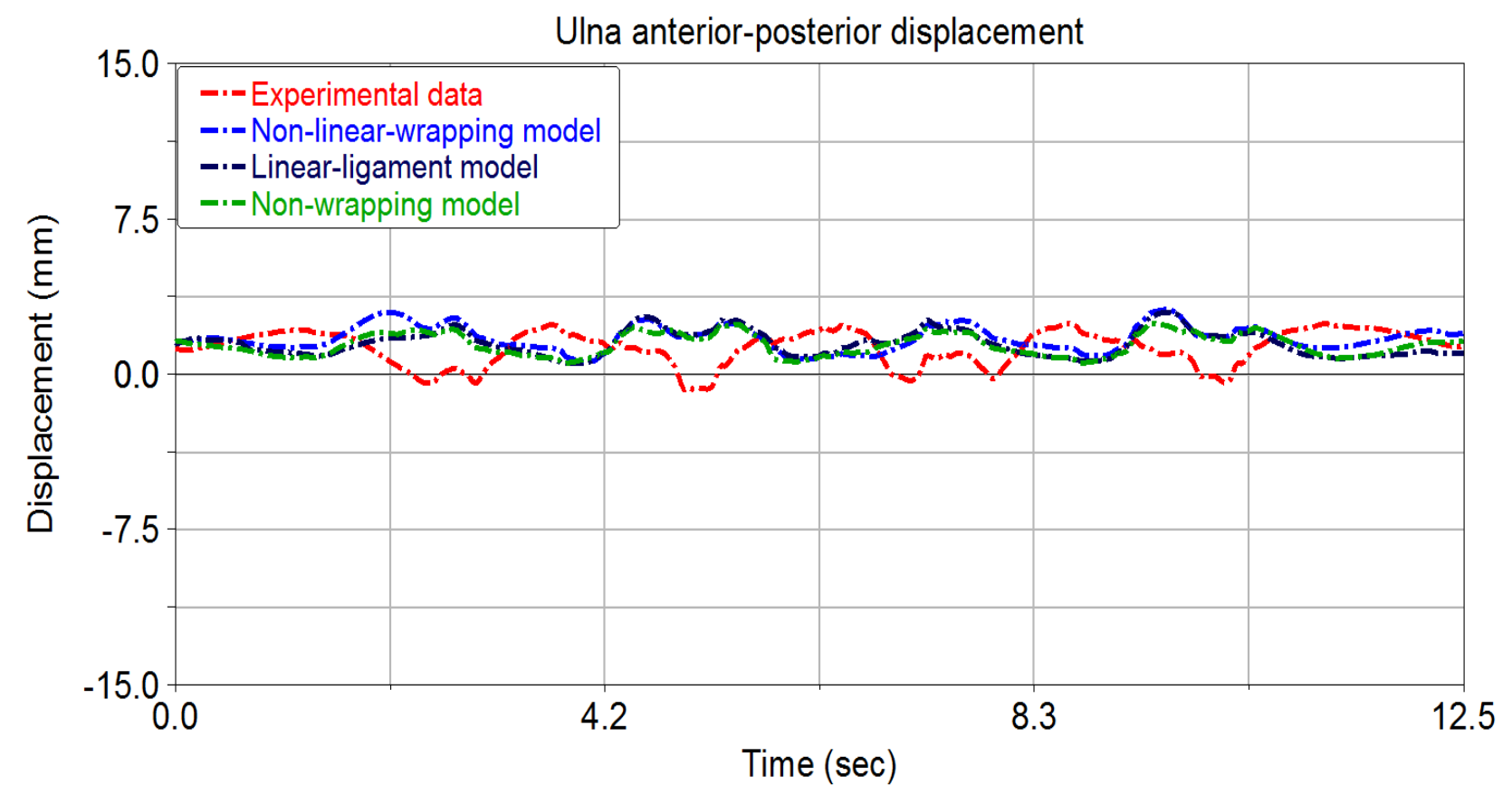

Figure 4.2. Anterior-posterior (A-P) displacement of the ulna coordinate system relative to the humerus coordinates for specimen 1 .

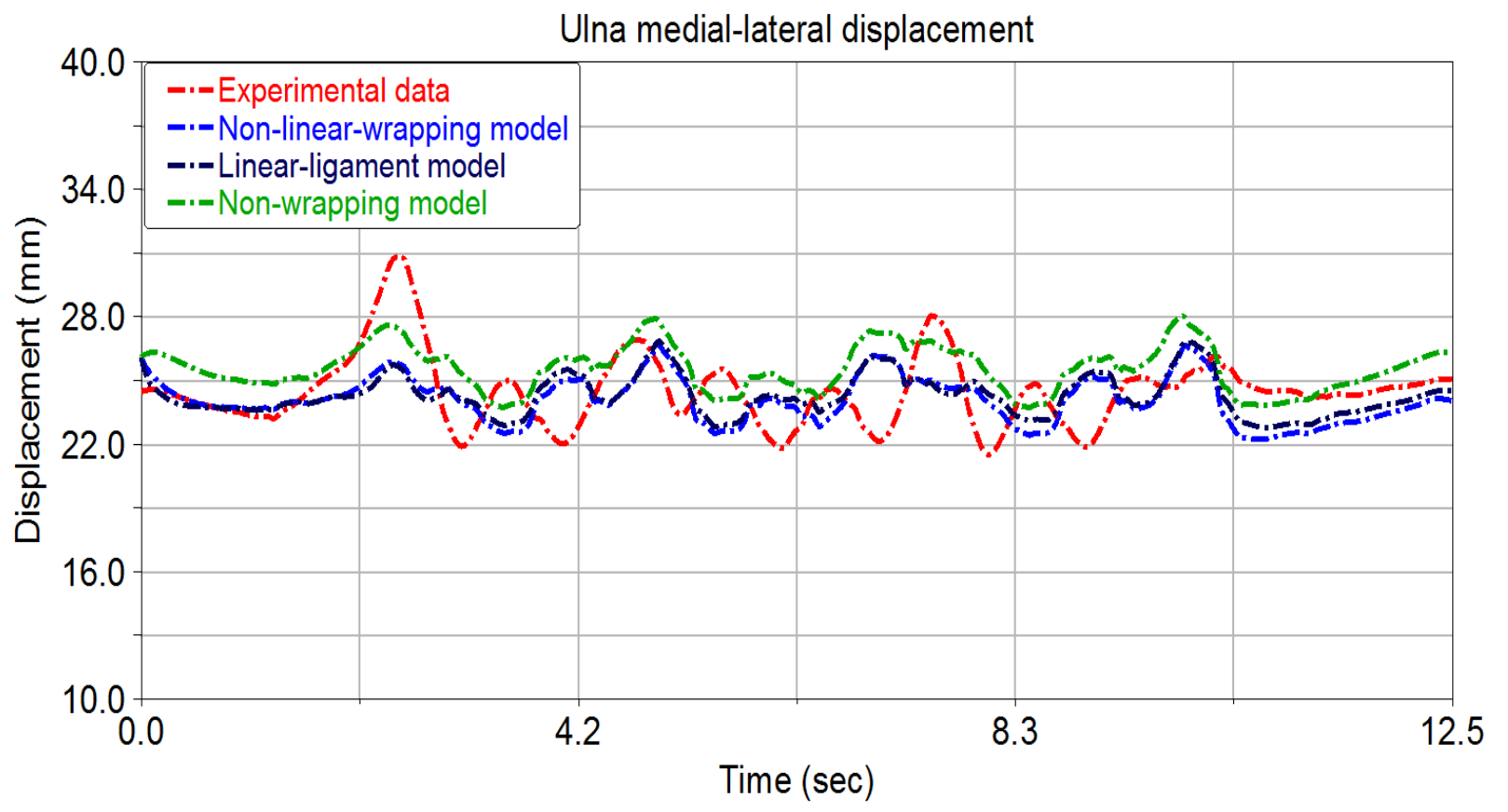

Figure 4.3. Medial-lateral (M-L) displacement of the ulna coordinate system relative to the humerus coordinates for specimen 1 . 
Figures 4.1-4.3 provide the S-I, A-P, and M-L displacements of ulna coordinate system relative to the humerus coordinate system respectively and presented in humerus coordinate. The magnitude of S-I and A-P displacement is comparatively small and reached a maximum range of $3 \mathrm{~mm}$. The value for $\mathrm{M}-\mathrm{L}$ displacement is greater than $\mathrm{S}-\mathrm{I}$ and $\mathrm{M}-\mathrm{L}$ displacement values and the range extend to about $8 \mathrm{~mm}$. The predicted results of ulna displacements are an average agreement with the experimental values, and the maximum RMS error (1.8 mm) occurs in M-L direction.

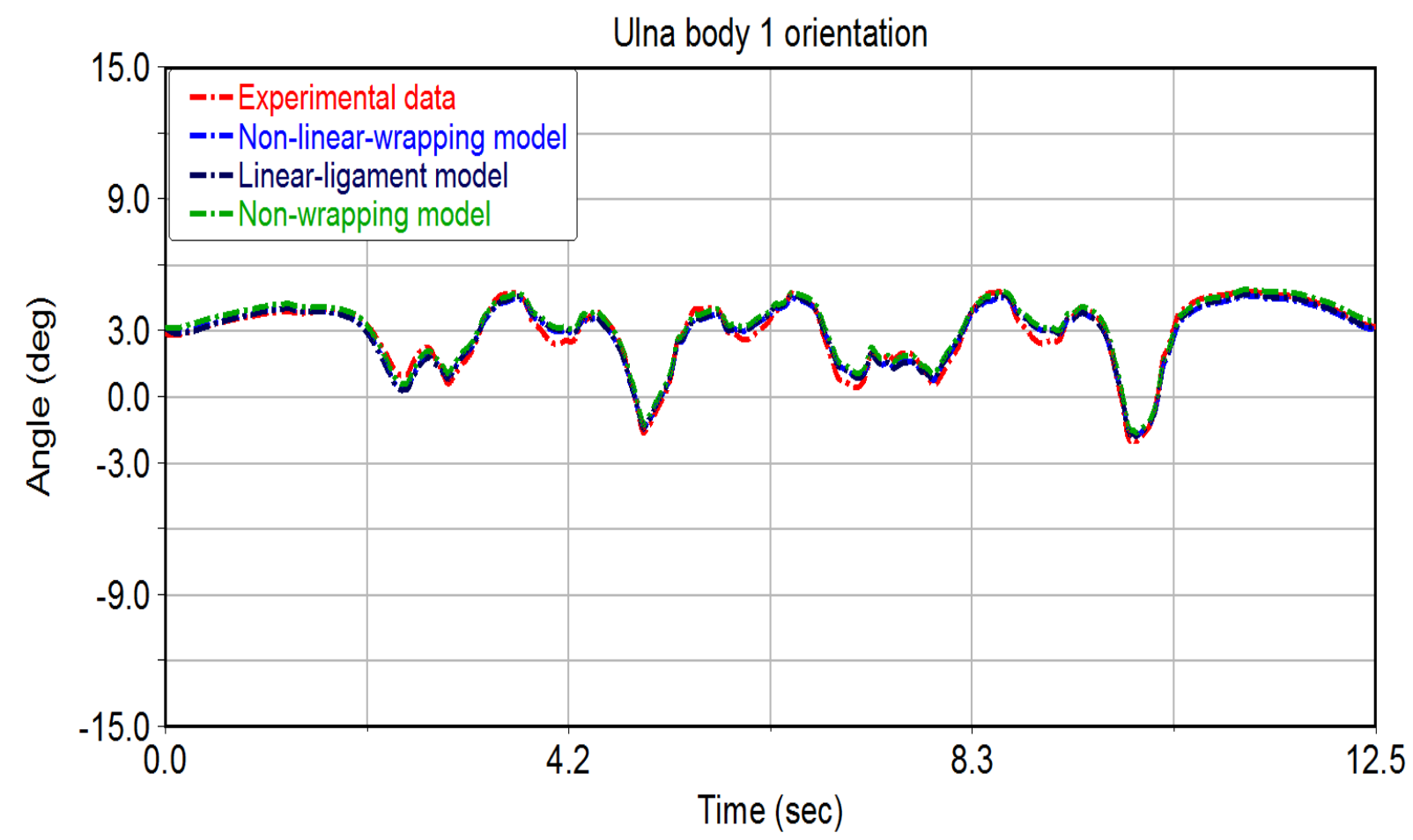

Figure 4.4. Ulna internal-external (I-E) rotation relative to the humerus coordinates for specimen 1 . 


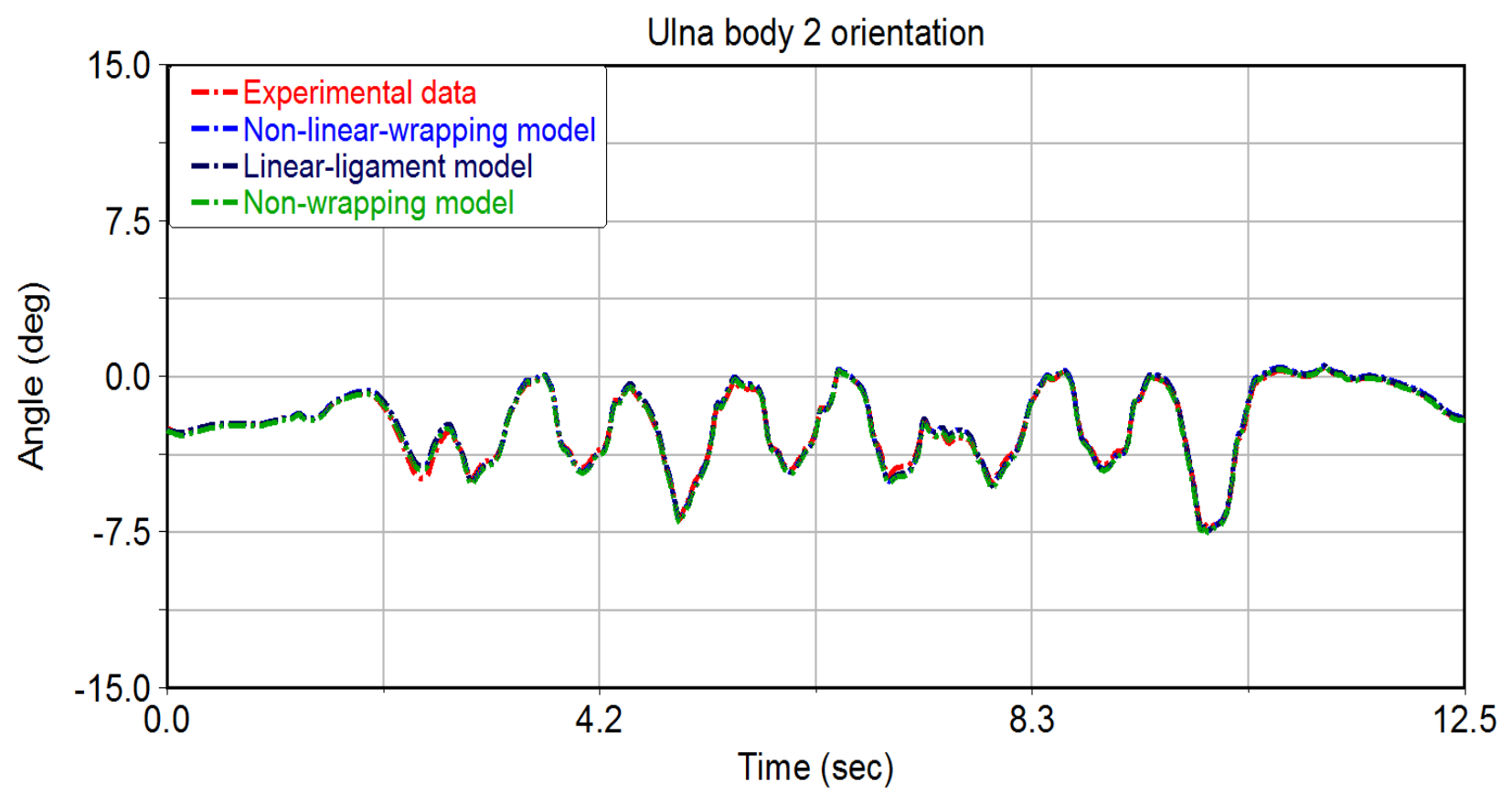

Figure 4.5. Ulna adduction-abduction (AD-AB duction) relative to the humerus coordinates for specimen 1 .

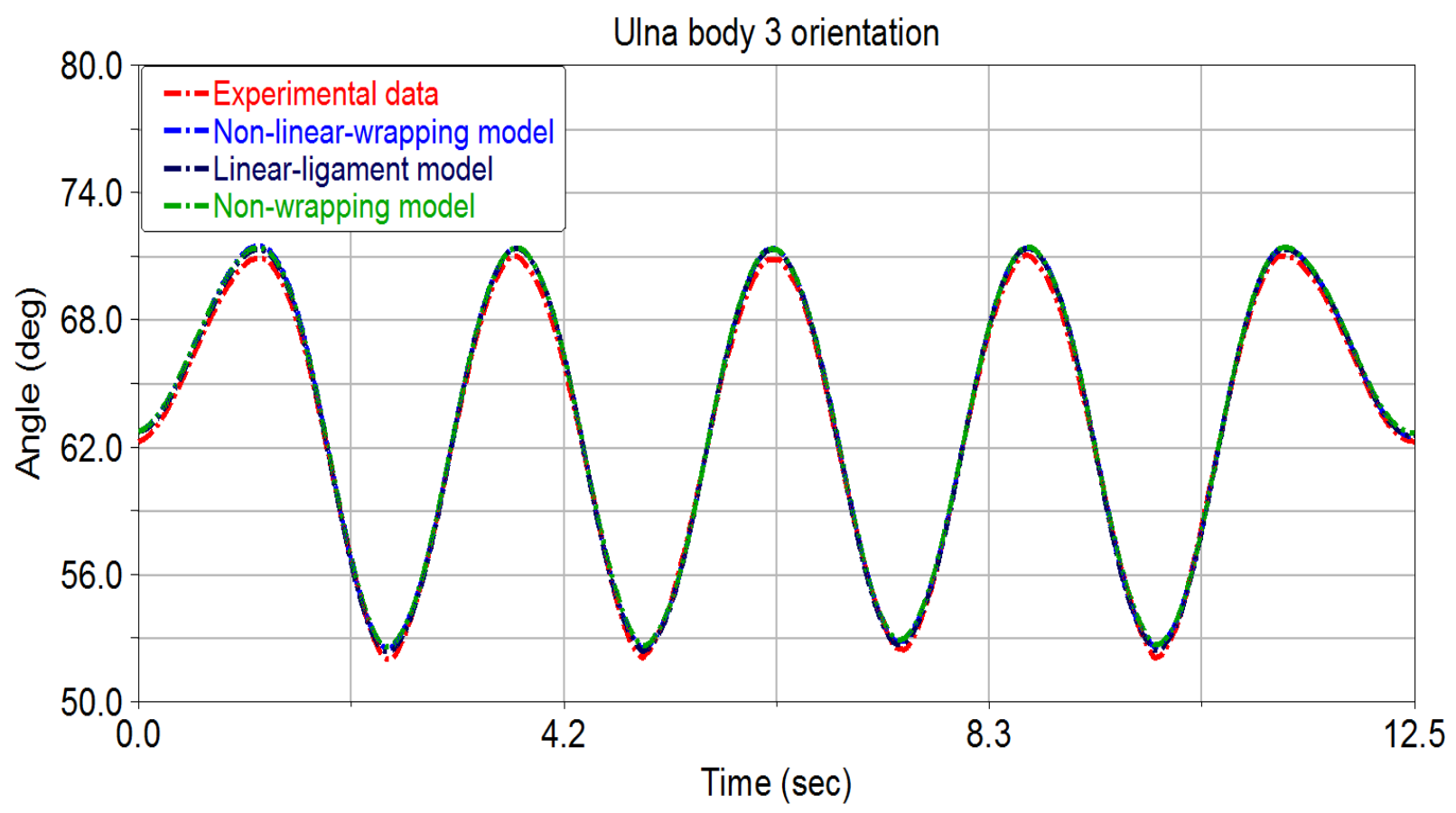

Figure 4.6. Ulna flexion-extension (F-E) relative to the humerus coordinates for specimen 1. 
The presented figures 4.4-4.6 shown above provide the comparison between the body $1,2,3$ orientations of ulna coordinate system relative to the humerus coordinate system about the $\mathrm{x}, \mathrm{y}$ and $\mathrm{z}$-axes of humerus coordinate system respectively. The orientation magnitudes are higher from their corresponding displacement magnitude. The body 3 orientation angle of the ulna relative to the humerus represents the flexion-extension (F-E) of the forearm. The value for F-E is the highest for the angles and ranged to nearly $20^{\circ}$. The angle values for body 1 and 2 are smaller than body 3 . The angel range was approximately $5^{\circ}$ for body 1 and $8^{\circ}$ for body 2 . The body 1 and 2 rotations represent the internal-external (I-E) and adductionabduction (AD-AB duction ) of the ulna relative to humerus respectively. As illustrated in the figures, the predicted results of ulna orientations are in a good agreement with the experimental values throughout the period of study.

\subsection{Radius kinematics}

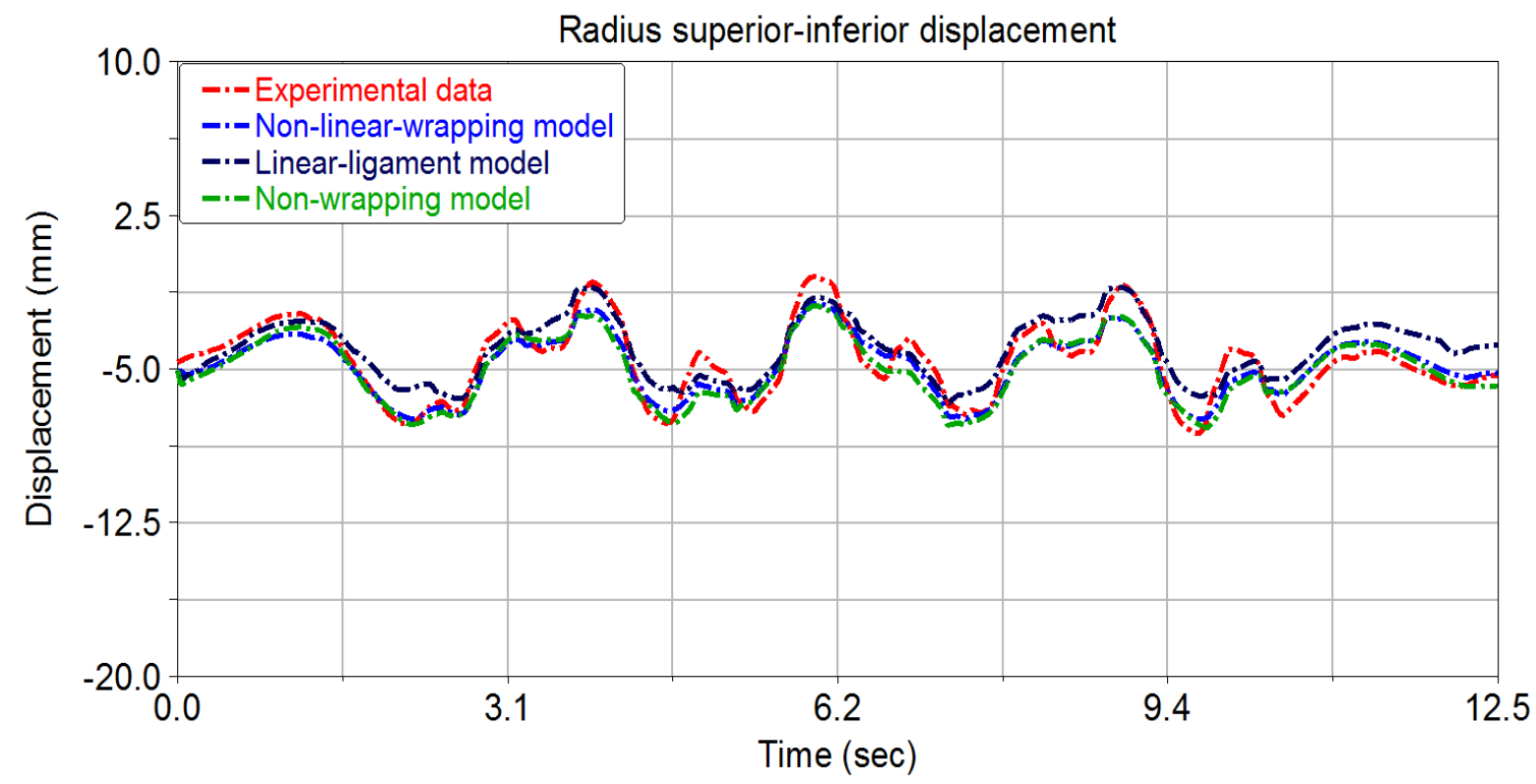

Figure 4.7. S-I displacement of the radius coordinate system relative to the humerus coordinates for specimen 1. 


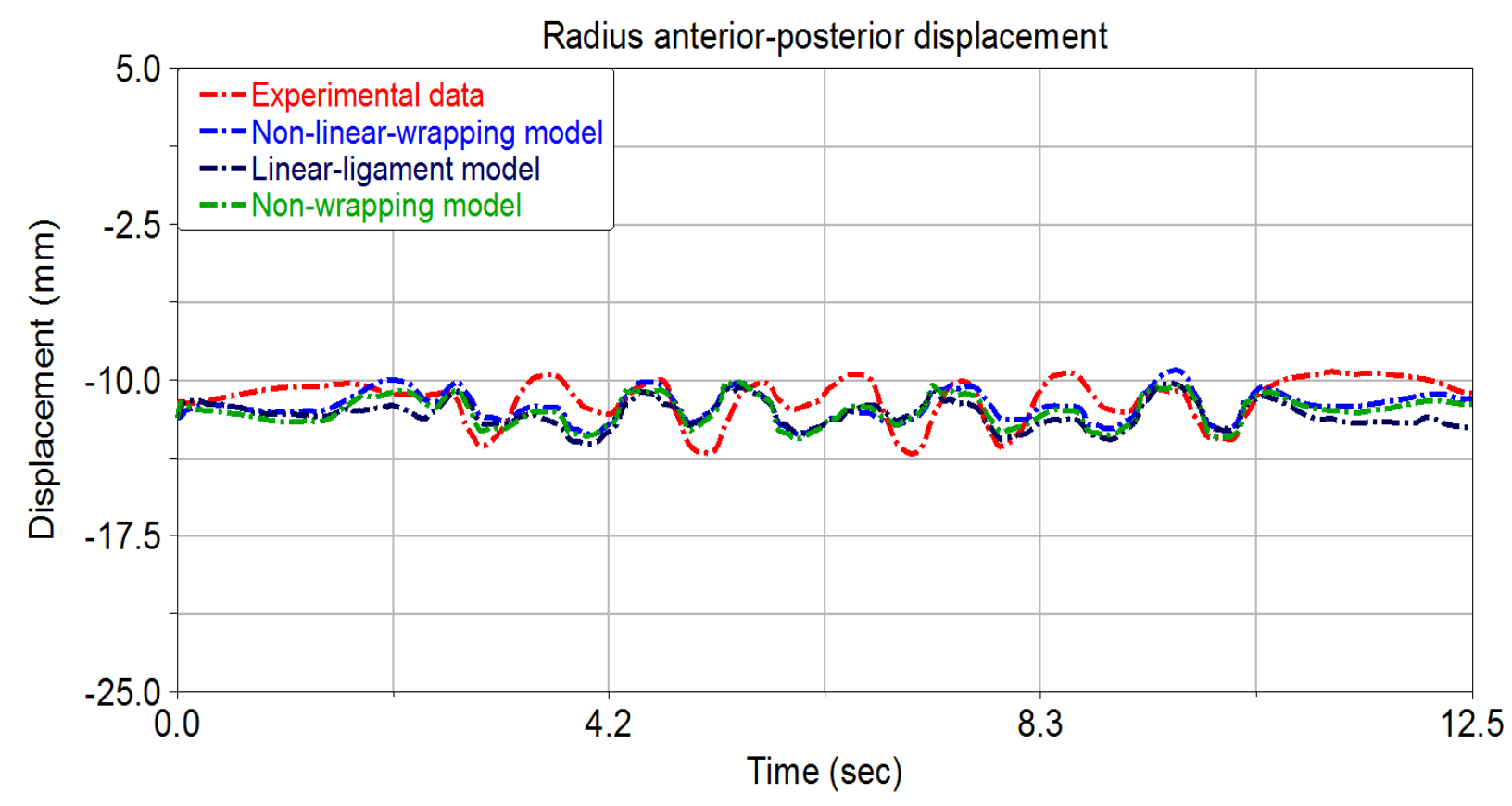

Figure 4.8. A-P displacement of the radius coordinate system relative to the humerus coordinates for specimen 1 .

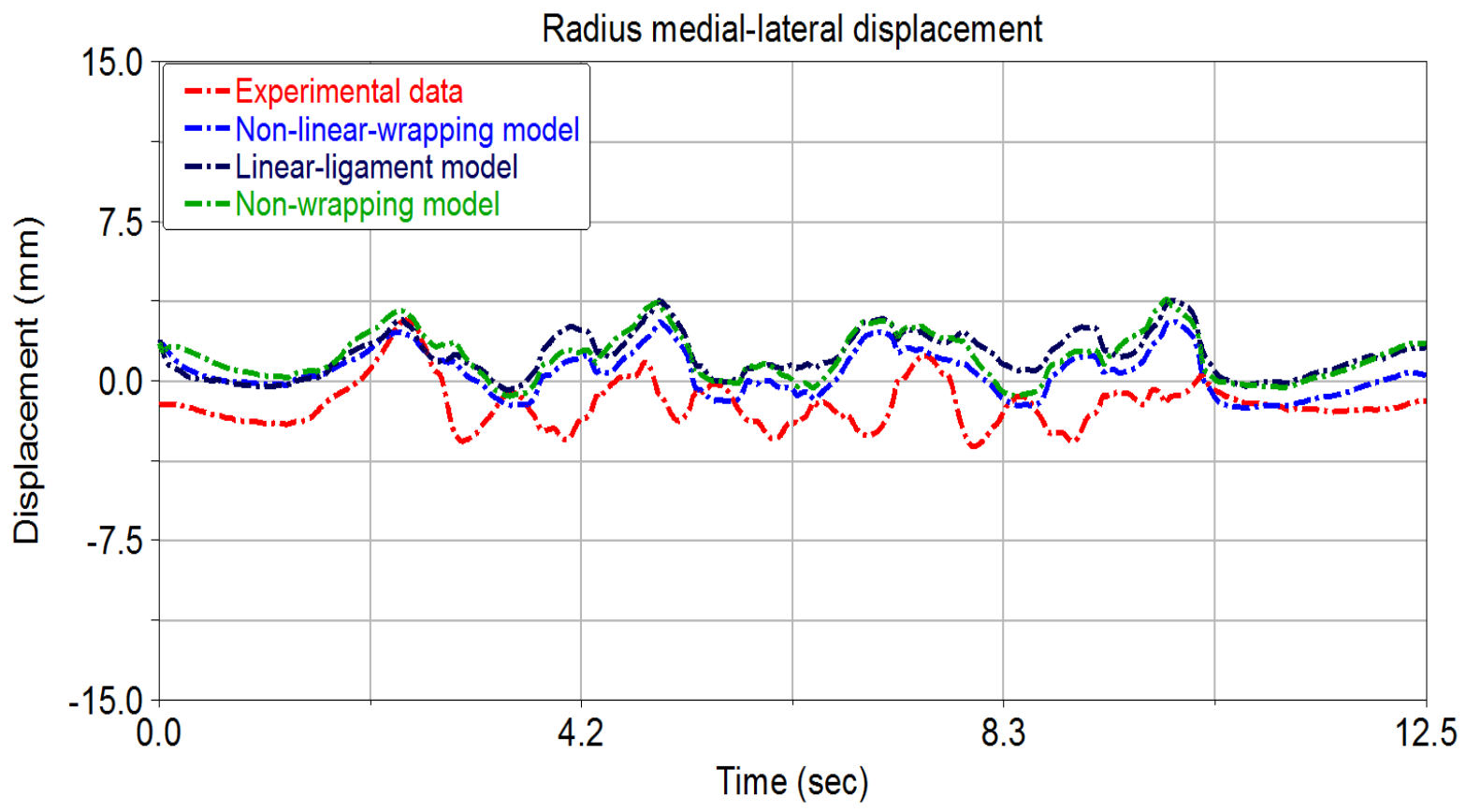

Figure 4.9. M-L displacement of the radius coordinate system relative to the humerus coordinates for specimen 1 . 
Figures 4.7-4.9 provides the S-I, A-P and M-L displacement of radius coordinate system relative to the humerus coordinate system respectively and presented in humerus coordinate system. The radius displacement practically shows the similar trends with the ulnar displacement as they are connected with the interosseous membrane and annular ligaments. However, since the radius has more laxity and less constraint than ulna, the magnitude of radius displacement is little bigger compare to ulna. The maximum value for M-L displacement is $7 \mathrm{~mm}$; and for S-I and A-P displacements are $6 \mathrm{~mm}$ and $4 \mathrm{~mm}$ respectively. It is observed from the figure that the predicted radius displacements have good proximity with the experimental values.

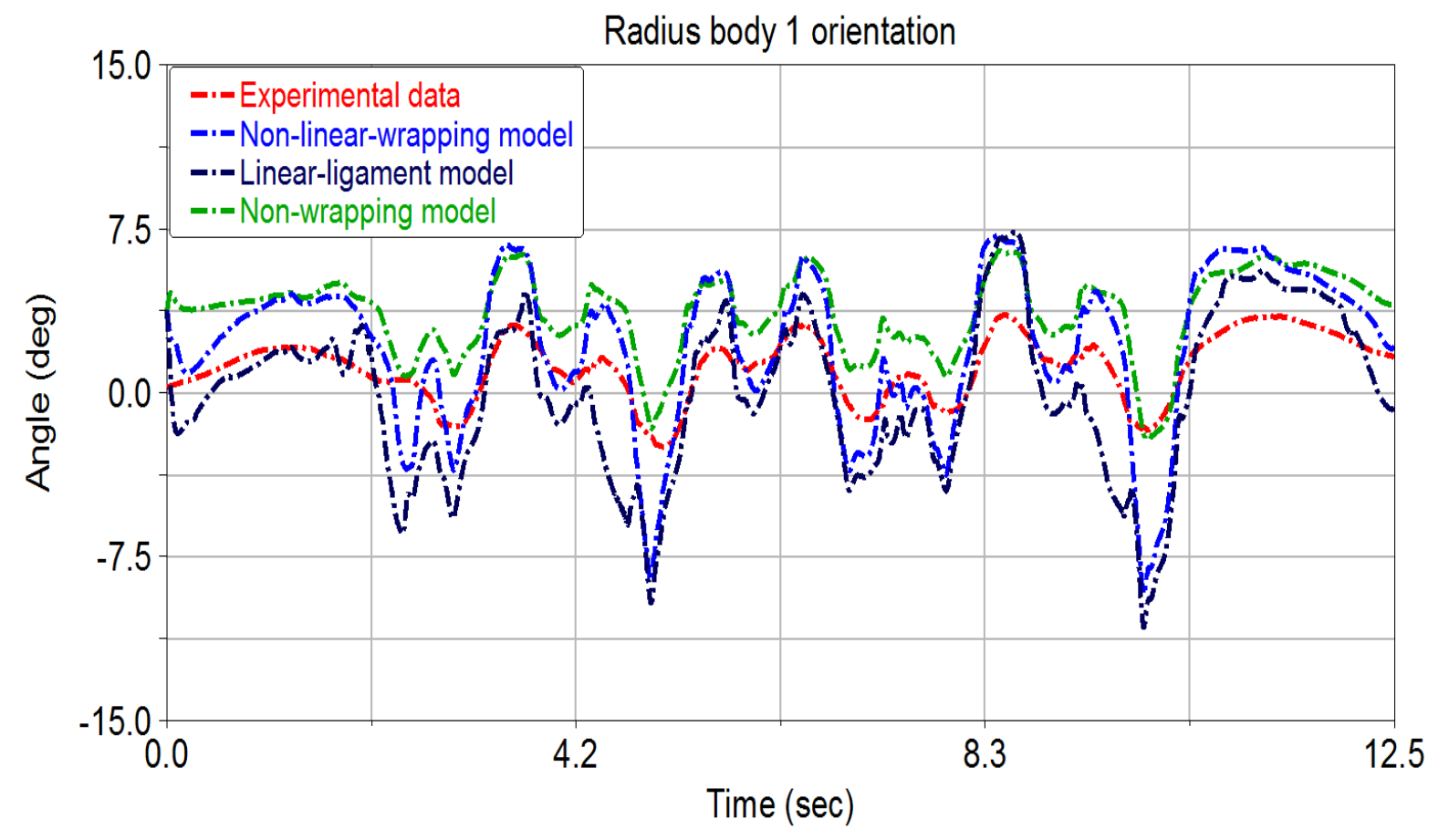

Figure 4.10. Radius I-E rotation relative to the humerus coordinates for specimen 1. 


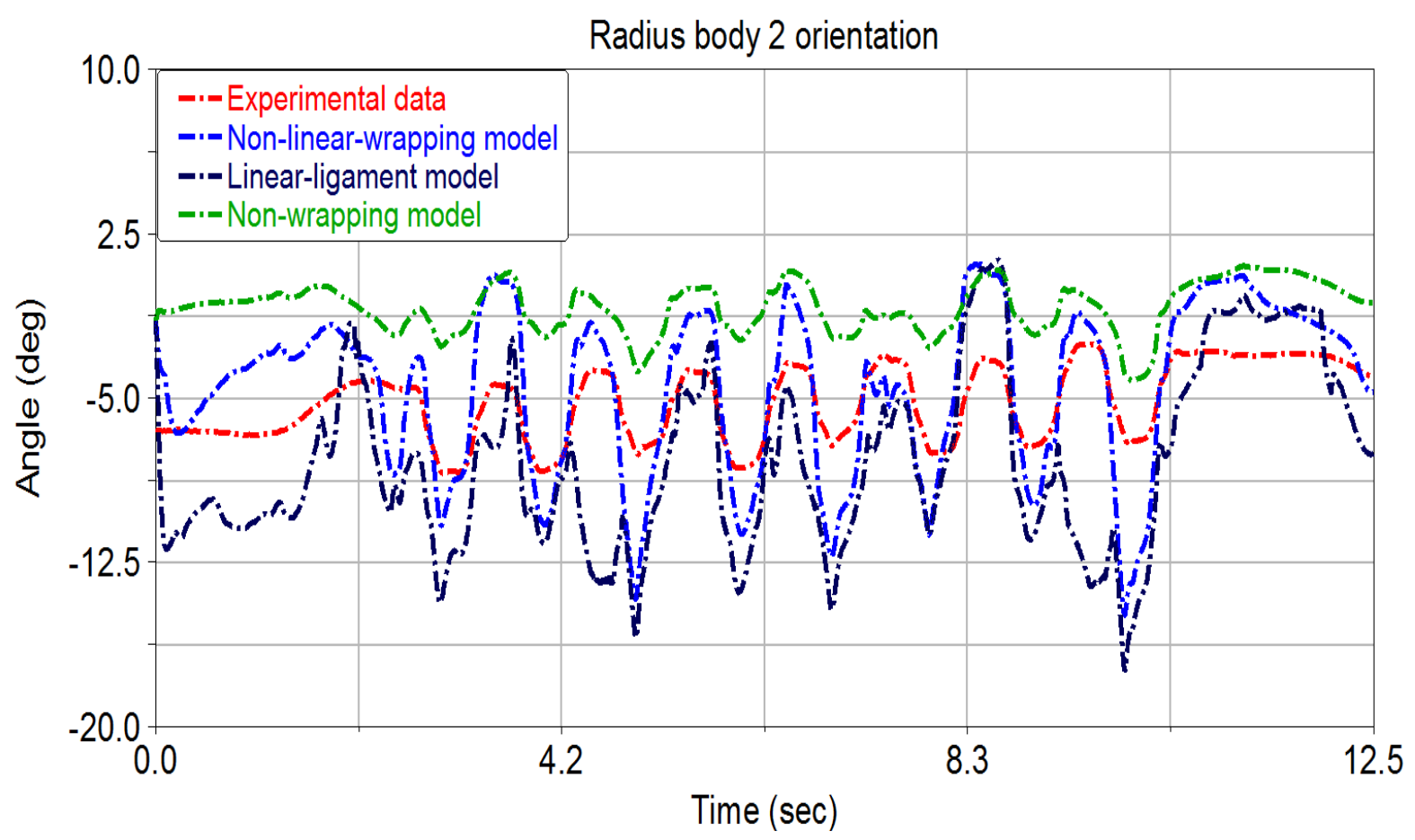

Figure 4.11. Radius I-E rotation relative to the humerus coordinates for specimen 1

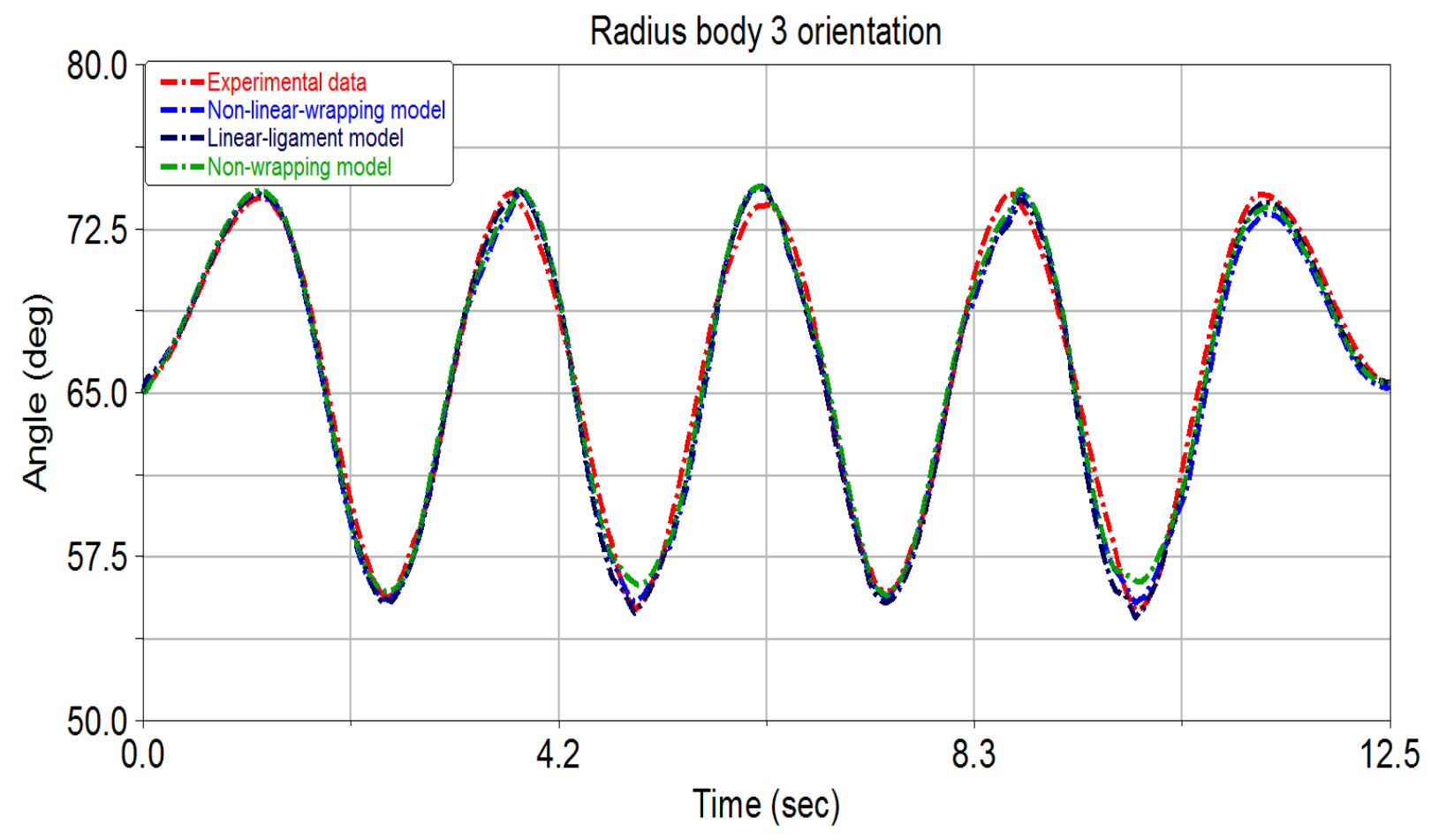

Figure 4.12. Radius AD-AB duction relative to the humerus coordinates for specimen 1. 
The body $1,2,3$ rotations of radius coordinate system relative to the humerus coordinate system as shown in figures 4.10-4.12 respectively represents the I-E rotation, AD$\mathrm{AB}$ duction, and $\mathrm{F}-\mathrm{E}$ of radius. Although the $\mathrm{I}-\mathrm{E}$ rotation and $\mathrm{AD}-\mathrm{AB}$ duction are somewhat deviated from the experimental observation, the F-E has a very good agreement with the experiment. The difference might be explained by a defected structure of cartilages that produced unpredicted contact surfaces and contact forces. As presented in the figures, the range of I-E rotation, $\mathrm{AD}-\mathrm{AB}$ duction, and $\mathrm{F}-\mathrm{E}$ are $12^{\circ}, 10^{\circ}$ and $20^{\circ}$ respectively.

\subsection{Triceps tendon forces}

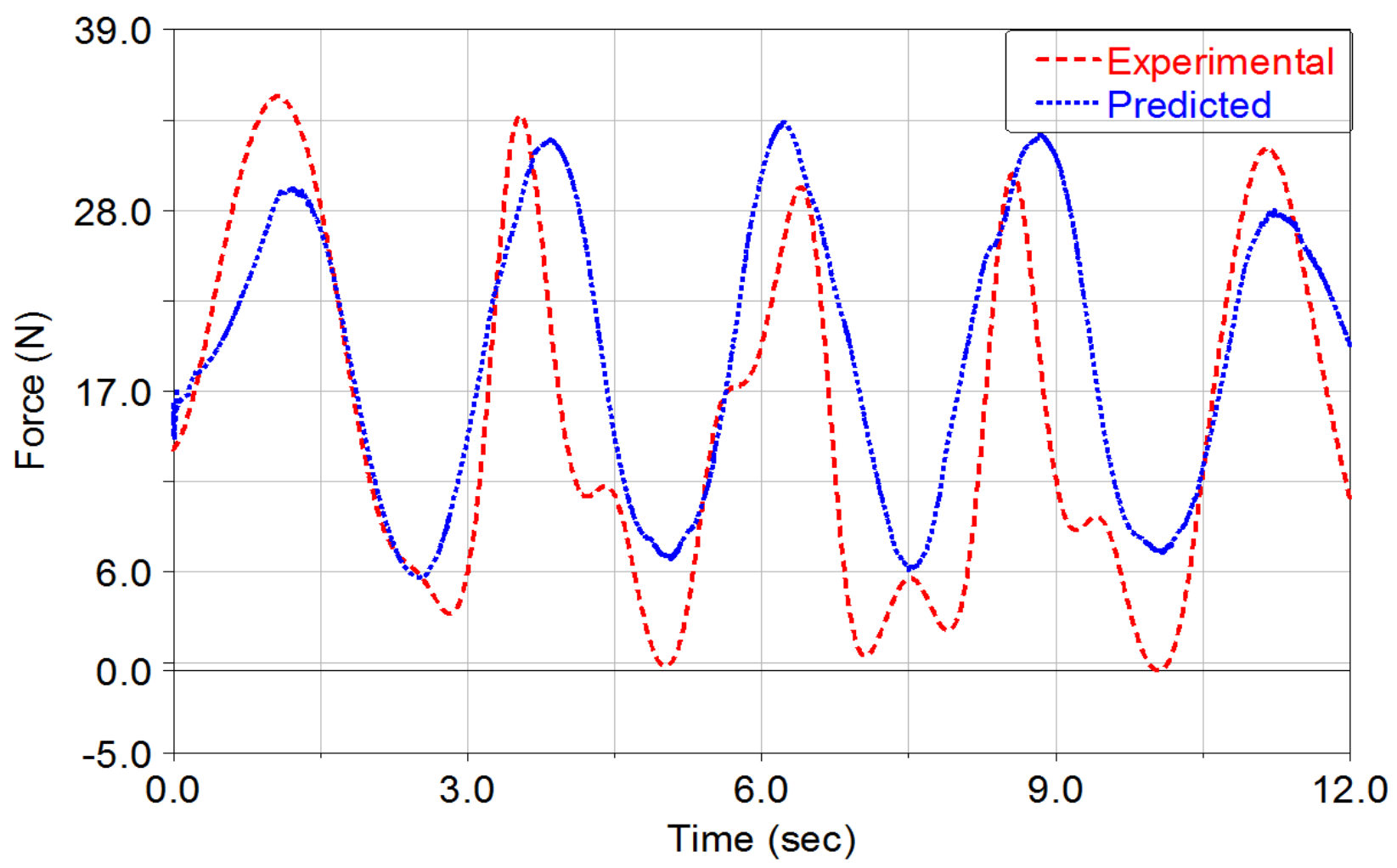

Figure 4.13. Comparison of triceps tendon force for specimen 1. 
The peak triceps tendon force observed from the figure 4.13 is about $33 \mathrm{~N}$. As illustrated in the figure, the triceps tendon was loaded when the elbow was flexing and unloaded when the elbow was extending. The experimental load cell signals were smoothed using a low-pass $2^{\text {nd }}$ order Butterworth filter with a cut-off frequency of $4 \mathrm{~Hz}$. The predicted force data has nearly similar trends to the experimental measured data although some difference was observed due to lack of accurate measurement of zero-load length and suture stiffness parameter. 


\section{CHAPTER 5}

\section{DISCUSSION}

The main purpose of this study was to develop and validate an anatomically correct subject specific 3D computational multibody model of the elbow joint complex. The models presented here were developed in the multibody framework that could be placed in neuromusucluloskeletal models of the upper arm. The models were validated by comparing the predicted bone kinematics and triceps tendon force to experimentally measured data from an identically loaded cadaver (Figs. 4.1 - 4.13). In order to quantify the model accuracy, the root mean square errors (RMS error) between model predictions and experimental measures were calculated. Overall, the RMS errors are small throughout the models that support a good agreement between the models and experiments.

A small improvement in kinematics compared to experimental measurement was observed when the lateral ulnar collateral and annular ligament were wrapped around the bones. Some additional reductions of RMS error were also acquired when a non-linear toe region was modeled in the ligament compared to models that had only a linear forcedisplacement relationship. Although these observations were not statistically significant (ANOVA $p$-value was greater than 0.05 ), this may suggest that ligament toe region and ligament wrapping should be included.

Review of the literature revealed several modeling approaches for the elbow joint (Buchanan et al., 1998; Garner \& Pandy, 2001; Gonzalez et al., 1999; Gonzalez et al., 1996; Holzbaur et al., 2005; Lemay \& Crago, 1996; Raikova, 1992; Schuind et al., 1991; Triolo et 
al., 2001). However, these models typically ignored the ligament contribution in joint modeling. Although some studies incorporated the ligament effect in the model (Fisk \& Wayne, 2009; Spratley \& Wayne, 2011), these studies ignored cartilages, ligament non-linear property and ligament wrapping around the bone. Modeling cartilage in the joints, wrapping the ligament around the bone, and incorporating the ligament non-linearity in the model is the unique work presented in this study.

The modeling of annular ligament wrapping benefits simulation of the radial head rotation. Modeling a circular path of for the annular ligament allows the radius to rotate inside the ligament similar to its physiological motion. Furthermore, it provides the attachment of radial collateral ligament with the annular ligament that provides the pathway for forces to be transmitted between the lateral epicondyle and radius/lateral ulna (Fisk, 2007). Along with the ligament contributions, the contact between the articular cartilages is essential to the elbow joint. The contact between the olecranon and olecranon fossa provides the elbow extension limit (Morrey, 2000). In addition, contact between the coronoid process and coronoid fossa provides significant effects on elbow flexion range of motion. Due to the multiple contact points between the coronoid process and coronoid fossa, the computational model here provides greater ulnohumeral contact force in elbow flexion (Fisk, 2007).

In the present study, the developed computational multibody model was able to represent flexion-extension associated with forearm pronation-supination accurately. The model was also able to persuasively predict joint function by providing a more detailed description of the underlying structures. Furthermore, this model allowed prediction of important biomechanical parameters that are difficult to measure in cadaver studies, such as contact and ligament forces. 
Although the results of this study are promising, differences still exist between the model and experimental investigations. In most cases, the largest values of RMS error occurred for the radius coordinate system relative to the humerus coordinate system. The biggest RMS errors for translation occurred in the medial-lateral direction for the radius relative to the humerus for specimen 2 and the value reached to $4.9 \mathrm{~mm}$ (Table 4.1). The largest rotation RMS error was $5.3^{0}$ adduction-abduction of the radius relative to the humerus for specimen 3 .

\subsection{Study limitations}

Multiple sources of modeling and experimental error exist that may have caused differences between model prediction and experimentally measured values. Due to poor quality of MRI, it was difficult to extract the real cartilage geometries. So, the articular cartilage geometries were assumed to have a uniform thickness over bone surfaces. But actual cartilage thickness is non-homogenous and varies over the bone. As a result, the modeled contact surface will differ from the actual contact surfaces that may affect contact magnitudes, locations and orientations, creating a source of modeling error. Furthermore, during experiment for the large rotations of the radius, the Optotrak cameras could lose sight of the ired markers, causing loss of kinematic data that affected the model validation. Additionally, the model was validated only by testing the resulting joint segment motions and triceps tendon force. Contact forces and pressures in the cadaver elbow joint were not directly measured. In addition, the models were validated with only three specimens. A larger sample size may help to make more generalized conclusions. 
The inability to predict stress and strain within the tissue is a major limitation of rigid multibody models. Predicting strain in cartilage and ligament tissues is important to understand tissue level degeneration and disease (Bei \& Fregly, 2004; Stylianou et al., 2012). The ligament path was chosen for annular ligaments as a rigid spline that may resist the translational movement of the radius. Including compliance contact instead of using point curve constraint in the ligament may improve the medial-lateral translation errors of the radius. Specimen 1 was disarticulated from the distal radioulnar joint, reducing constraints for the radius and introducing unexpected movements. However, these limitations had been overcome by using the steel dynamic compression plate for specimen 2 and 3 . Finally, the biaxial mechanical tester had a limited range of motion $(50 \mathrm{~mm})$ that producing small changes in flexion angle during experimental testing. A larger range of motion evaluating the elbow joint is important to understand ligament characteristics.

\subsection{Future work}

Various future works could be explored for this model such as; adding non-uniform discrete cartilage, more ligament wrapping, incorporating ligament viscoelastic properties, adding more muscle force and muscle contractile properties, and build a full musculoskeletal model of upper extremities. Discretizing the cartilage would make it possible to predict contact pressures in the cartilage during loading profiles. As a result, valuable insight about the joint functions and developing better surgical technique for cartilage deficiency could be attainable. To get a more generalized idea about model prediction and to intensify the model validation, a larger sample size would be recommended. It is also recommended to use the large value of flexion-extension to understand the ligament contribution in joint stability. 
This modeling work is the first step in developing a musculoskeletal model of the elbow joint. The developed model will then be used for subject specific full musculoskeletal movement simulations of the upper-extremity.

\subsection{Conclusion}

The computational multibody model has been developed and validated by comparing the simulated results with the experimental investigations. The models can predict some parameters that are difficult to investigate experimentally, such as forces within ligaments and contact forces between cartilages covered bones. While these results are promising, further refinements of model parameters may increase its accuracy and applicability. The models presented here were developed in the multibody framework that will be finally incorporate into the neuro-musucluloskeletal models of the upper arm. 
KINEMATICS COMPARISON FOR SPECIMEN 2 AND 3
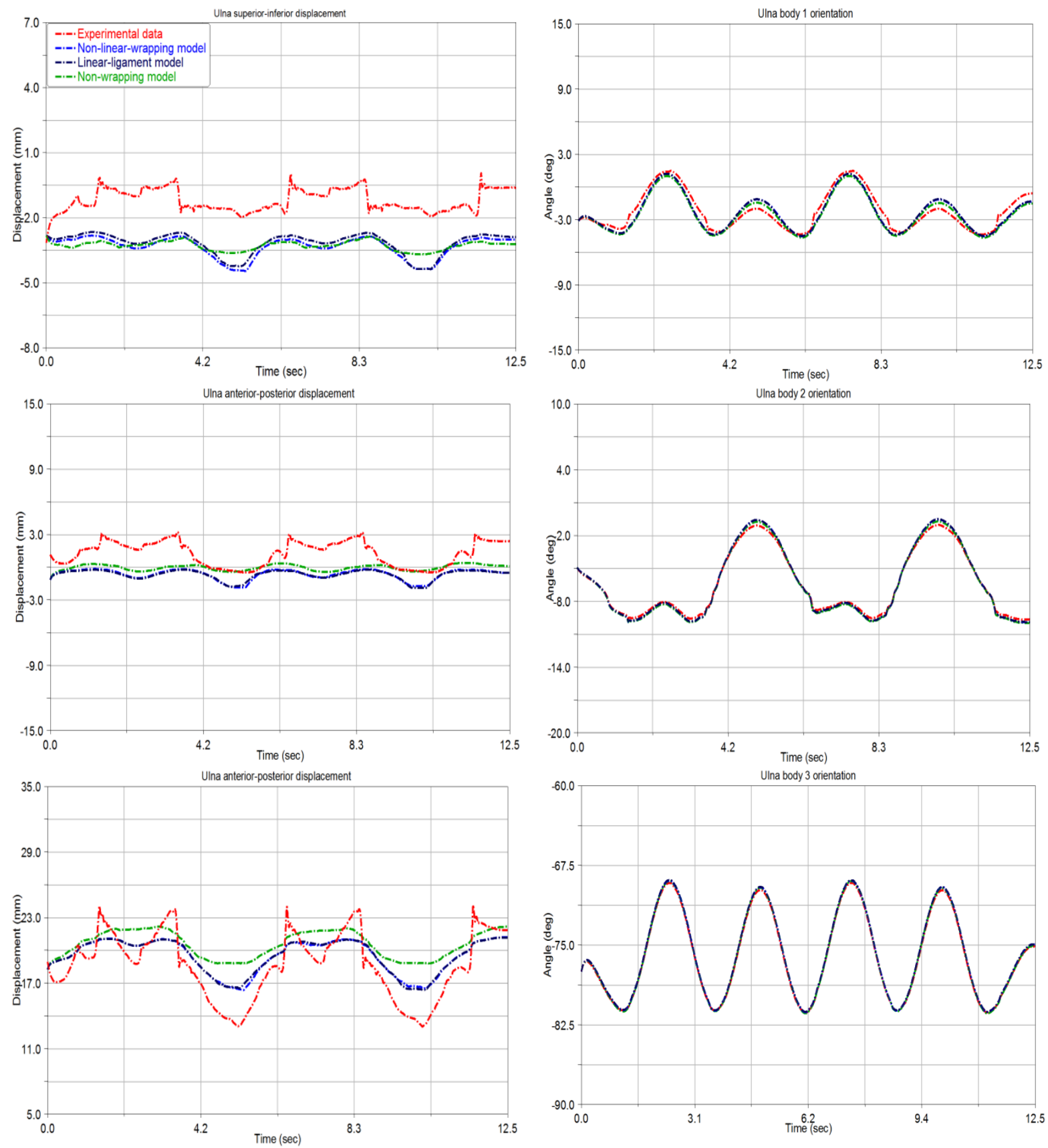

Figure A.1. Measured and predicted displacement and rotation of the ulna coordinate system relative to the humerus coordinate system for specimen 2 . 

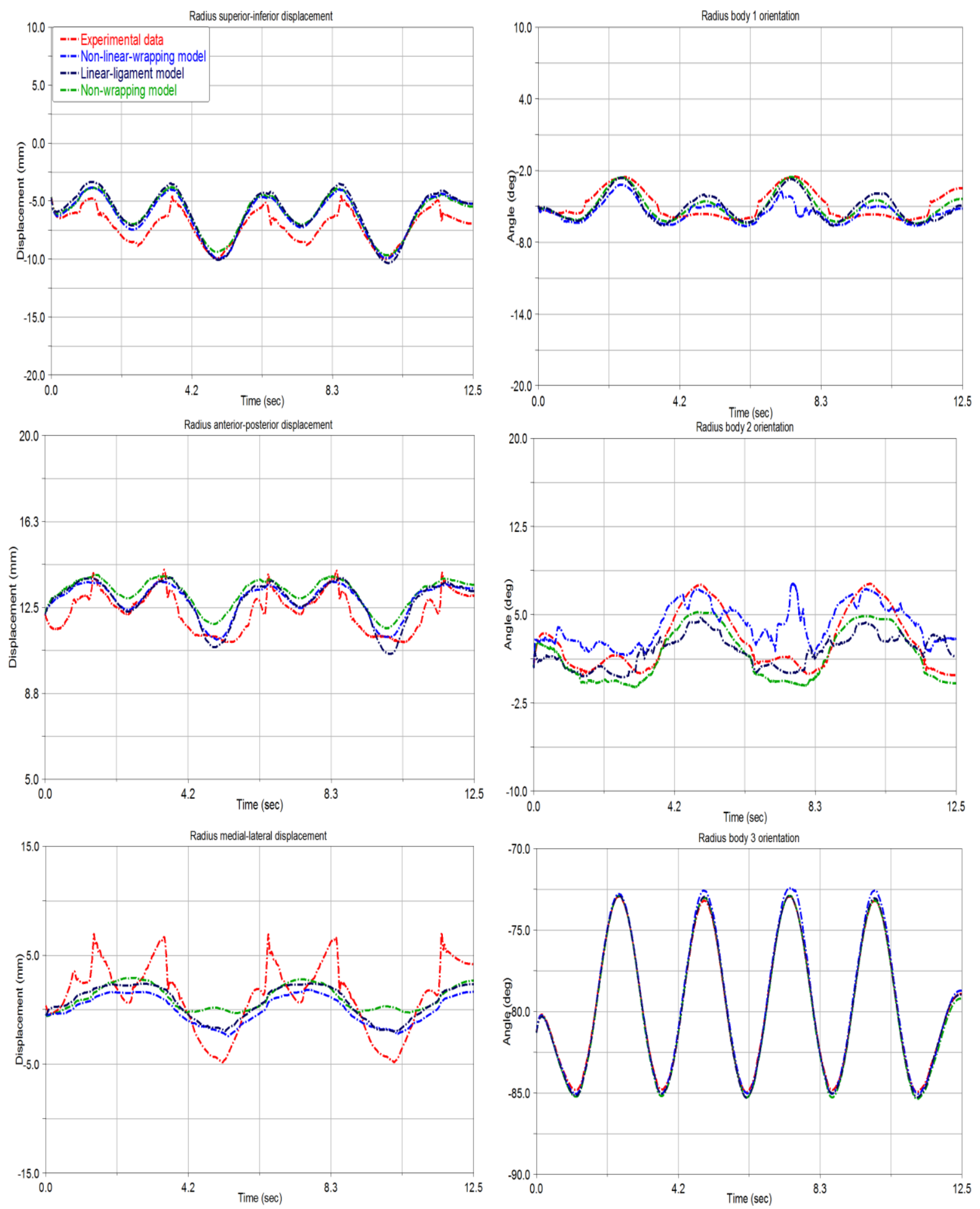

Figure A.2 Measured and predicted displacement and rotation of the radius coordinate system relative to the humerus coordinate system for specimen 2 . 

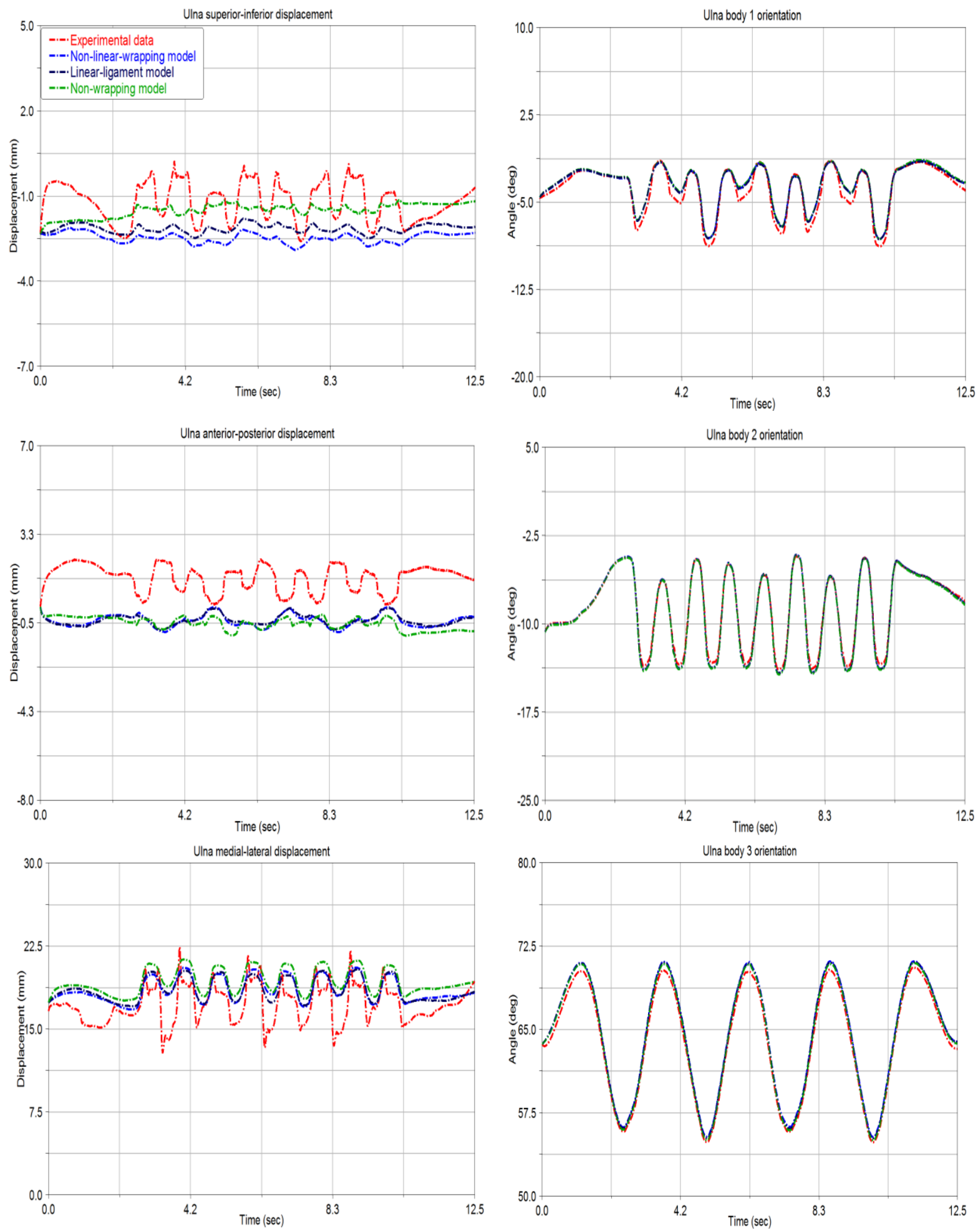

Figure A.3. Measured and predicted displacement and rotation of the ulna coordinate system relative to the humerus coordinate system for specimen 3 . 

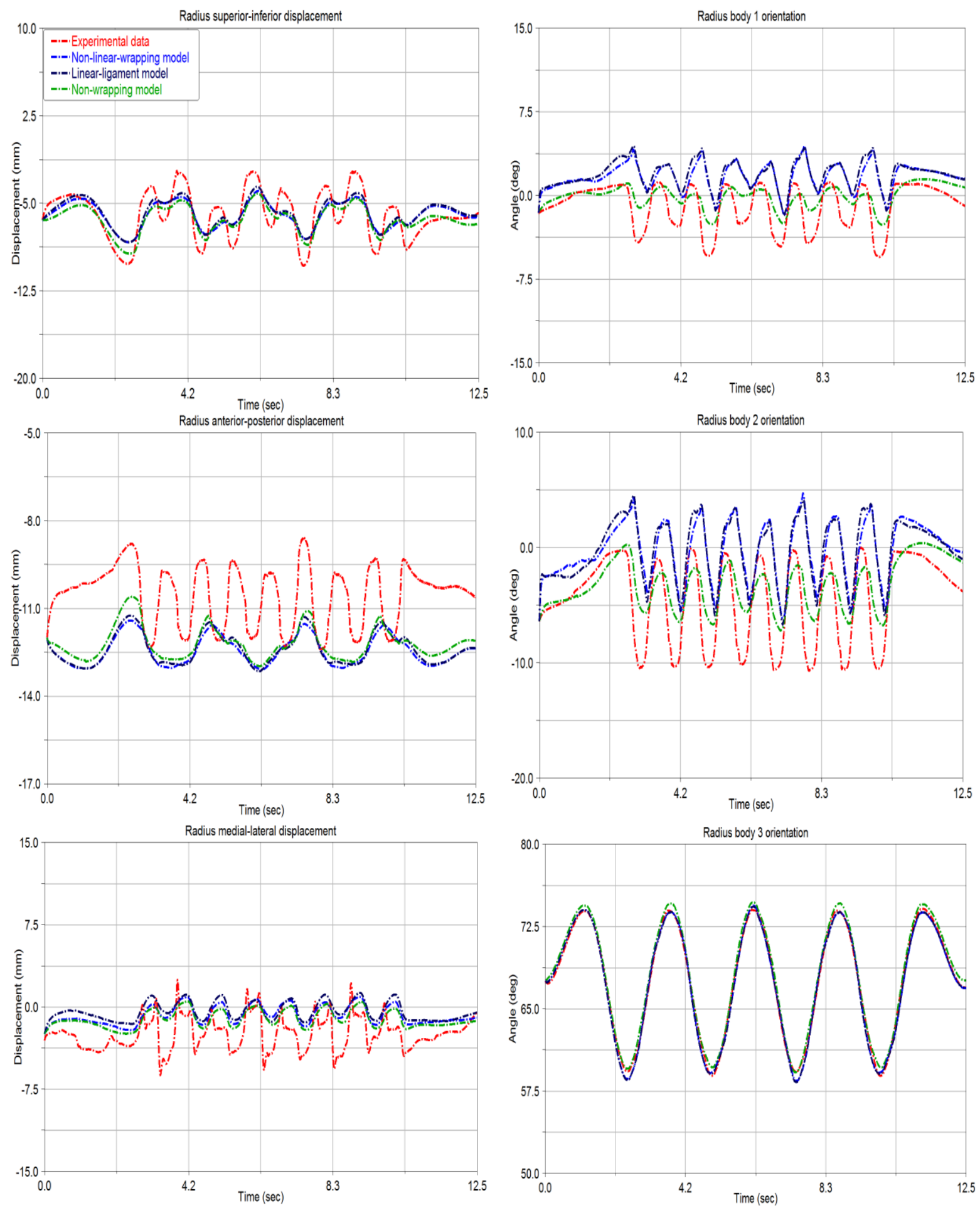

Figure A.4. Measured and predicted displacement and rotation of the radius coordinate system relative to the humerus coordinate system for specimen 3 . 


\section{REFERENCES}

Alcid, J. G., Ahmad, C. S., \& Lee, T. Q. (2004). Elbow anatomy and structural biomechanics. Clin Sports Med, 23(4), 503-517, vii. doi: 10.1016/j.csm.2004.06.008

Amis, A., Dowson, D., \& Wright, V. (1979). Muscle strengths and musculoskeletal geometry of the upper limb. Engineering in Medicine, 8(1), 41-48.

An, K. N. (2005). Kinematics and constraint of total elbow arthroplasty. J Shoulder Elbow Surg, 14(1 Suppl S), 168S-173S. doi: 10.1016/j.jse.2004.09.035

Anderson, F. C., \& Pandy, M. G. (2001). Dynamic optimization of human walking. $J$ Biomech Eng, 123(5), 381-390.

Arnold, A. S., \& Delp, S. L. (2001). Rotational moment arms of the medial hamstrings and adductors vary with femoral geometry and limb position: implications for the treatment of internally rotated gait. J Biomech, 34(4), 437-447. doi: 10.1016/s00219290(00)00232-3

Barker, T. M., Kirtley, C., \& Ratanapinunchai, J. (1997). Calculation of multi-segment rigid body joint dynamics using MATLAB. Proc Inst Mech Eng H, 211(6), 483-487.

Bei, Y., \& Fregly, B. J. (2004). Multibody dynamic simulation of knee contact mechanics. Med Eng Phys, 26(9), 777-789. doi: 10.1016/j.medengphy.2004.07.004

Benham, M. P., Wright, D. K., \& Bibb, R. (2001). Modelling soft tissue for kinematic analysis of multi-segment human body models. Biomed Sci Instrum, 37, 111-116.

Blankevoort, L., Kuiper, J. H., Huiskes, R., \& Grootenboer, H. J. (1991). Articular contact in a three-dimensional model of the knee. J Biomech, 24(11), 1019-1031.

Bloemker, K. H., Guess, T. M., Maletsky, L., \& Dodd, K. (2012). Computational knee ligament modeling using experimentally determined zero-load lengths. Open Biomed Eng J, 6, 33-41. doi: 10.2174/1874230001206010033 
Buchanan, T. S., Delp, S. L., \& Solbeck, J. A. (1998). Muscular resistance to varus and valgus loads at the elbow. J Biomech Eng, 120(5), 634-639.

Chao, E. Y., Armiger, R. S., Yoshida, H., Lim, J., \& Haraguchi, N. (2007). Virtual Interactive Musculoskeletal System (VIMS) in orthopaedic research, education and clinical patient care. J Orthop Surg Res, 2, 2. doi: 10.1186/1749-799x-2-2

Chaudhari, A. M., \& Andriacchi, T. P. (2006). The mechanical consequences of dynamic frontal plane limb alignment for non-contact ACL injury. J Biomech, 39(2), 330-338. doi: 10.1016/j.jbiomech.2004.11.013

Chuang, L. L., Wu, C. Y., Lin, K. C., \& Lur, S. Y. (2012). Quantitative mechanical properties of the relaxed biceps and triceps brachii muscles in patients with subacute stroke: a reliability study of the myoton-3 myometer. Stroke Res Treat, 2012, 617694. doi: $10.1155 / 2012 / 617694$

Cohen, M. S., \& Bruno, R. J. (2001). The collateral ligaments of the elbow: anatomy and clinical correlation. Clin Orthop Relat Res(383), 123-130.

Cohen, Z. A., Henry, J. H., McCarthy, D. M., Mow, V. C., \& Ateshian, G. A. (2003). Computer simulations of patellofemoral joint surgery. Patient-specific models for tuberosity transfer. Am J Sports Med, 31(1), 87-98.

Currier, D. P. (1972). Maximal isometric tension of the elbow extensors at varied positions. I. Assessment by cable tensiometer. Phys Ther, 52(10), 1043-1049.

de Haan, J., Schep, N. W., Eygendaal, D., Kleinrensink, G. J., Tuinebreijer, W. E., \& den Hartog, D. (2011). Stability of the elbow joint: relevant anatomy and clinical implications of in vitro biomechanical studies. Open Orthop J, 5, 168-176. doi: $10.2174 / 1874325001105010168$

Degreef, I., \& De Smet, L. (2011). The arthroscopic ulnohumeral arthroplasty: from miniopen to arthroscopic surgery. Minim Invasive Surg, 2011, 798084. doi: $10.1155 / 2011 / 798084$

Delp, S. L., \& Loan, J. P. (1995). A graphics-based software system to develop and analyze models of musculoskeletal structures. Comput Biol Med, 25(1), 21-34. 
Donahue, T. L., Hull, M. L., Rashid, M. M., \& Jacobs, C. R. (2002). A finite element model of the human knee joint for the study of tibio-femoral contact. $J$ Biomech Eng, 124(3), 273-280.

Fernandez, J. W., \& Pandy, M. G. (2006). Integrating modelling and experiments to assess dynamic musculoskeletal function in humans. Exp Physiol, 91(2), 371-382. doi: 10.1113/expphysiol.2005.031047

Ferreira, L. M., (2011). Development of an Active Elbow Motion Simulator and Coordinate Systems to Evaluate Kinematics in Multiple Positions. Paper 84.

Ferreira, L. M., King, G. J., \& Johnson, J. A. (2011). Motion-derived coordinate systems reduce inter-subject variability of elbow flexion kinematics. J Orthop Res, 29(4), 596601. doi: $10.1002 /$ jor.21278

Fisk, J. P. (2007). Development and validation of a computational musculoskeletal model of the elbow joint. Virginia Commonwealth University. Richmond, Virginia

Fisk, J. P., \& Wayne, J. S. (2009). Development and validation of a computational musculoskeletal model of the elbow and forearm. Ann Biomed Eng, 37(4), 803-812. doi: 10.1007/s10439-009-9637-x

Freund, J., \& Takala, E. P. (2001). A dynamic model of the forearm including fatigue. $J$ Biomech, 34(5), 597-605.

Fuss, F. K. (1991). The ulnar collateral ligament of the human elbow joint. Anatomy, function and biomechanics. J Anat, 175, 203-212.

Garner, B. A., \& Pandy, M. G. (2001). Musculoskeletal model of the upper limb based on the visible human male dataset. Comput Methods Biomech Biomed Engin, 4(2), 93-126. doi: $10.1080 / 10255840008908000$

Gavronski, G., Veraksits, A., Vasar, E., \& Maaroos, J. (2007). Evaluation of viscoelastic parameters of the skeletal muscles in junior triathletes. Physiol Meas, 28(6), 625-637. doi: $10.1088 / 0967-3334 / 28 / 6 / 002$

Giddings, V. L., Beaupre, G. S., Whalen, R. T., \& Carter, D. R. (2000). Calcaneal loading during walking and running. Med Sci Sports Exerc, 32(3), 627-634. 
Gonzalez, R. V., Abraham, L. D., Barr, R. E., \& Buchanan, T. S. (1999). Muscle activity in rapid multi-degree-of-freedom elbow movements: solutions from a musculoskeletal model. Biol Cybern, 80(5), 357-367.

Gonzalez, R. V., Andritsos, M. J., Barr, R. E., \& Abraham, L. D. (1993). Comparison of experimental and predicted muscle activation patterns in ballistic elbow joint movements. Biomed Sci Instrum, 29, 9-16.

Gonzalez, R. V., Hutchins, E. L., Barr, R. E., \& Abraham, L. D. (1996). Development and evaluation of a musculoskeletal model of the elbow joint complex. J Biomech Eng, $118(1), 32-40$.

Guess, T. M. (2012). Forward dynamics simulation using a natural knee with menisci in the multibody framework. Multibody System Dynamics, 28(1-2), 37-53. doi: 10.1007/s11044-011-9293-4

Guess, T. M., Liu, H., Bhashyam, S., \& Thiagarajan, G. (2013). A multibody knee model with discrete cartilage prediction of tibio-femoral contact mechanics. Comput Methods Biomech Biomed Engin, 16(3), 256-270. doi: $10.1080 / 10255842.2011 .617004$

Guess, T. M., \& Stylianou, A. (2012). Simulation of anterior cruciate ligament deficiency in a musculoskeletal model with anatomical knees. Open Biomed Eng J, 6, 23-32. doi: $10.2174 / 1874230001206010023$

Guess, T. M., Thiagarajan, G., Kia, M., \& Mishra, M. (2010). A subject specific multibody model of the knee with menisci. Med Eng Phys, 32(5), 505-515. doi: 10.1016/j.medengphy.2010.02.020

Hirokawa, S. (1991). Three-dimensional mathematical model analysis of the patellofemoral joint. J Biomech, 24(8), 659-671.

Holzbaur, K. R., Murray, W. M., \& Delp, S. L. (2005). A model of the upper extremity for simulating musculoskeletal surgery and analyzing neuromuscular control. Ann Biomed Eng, 33(6), 829-840. 
Hotchkiss, R. N., An, K. N., Sowa, D. T., Basta, S., \& Weiland, A. J. (1989). An anatomic and mechanical study of the interosseous membrane of the forearm: pathomechanics of proximal migration of the radius. J Hand Surg Am, 14(2 Pt 1), 256-261.

Hunt, K. H., \& Crossley, F. R. E. (1975). Coefficient of Restitution Interpreted as Damping in Vibroimpact. Journal of Applied Mechanics, 42(2), 440. doi: 10.1115/1.3423596

Hutchins, E. L., Gonzalez, R. V., \& Barr, R. E. (1993). Comparison of experimental and analytical torque-angle relationships of the human elbow joint complex. Biomed Sci Instrum, 29, 17-24.

Identify the muscles crossing the elbow joint. (2013). Retrieved 11/5/2013, from http://www.usi.edu/science/biology/mkhopper/2401/01Ex14MuscleAnatomy/IDElbo wJoint.htm

Iwasaki, N., Genda, E., Barrance, P. J., Minami, A., Kaneda, K., \& Chao, E. Y. (1998). Biomechanical analysis of limited intercarpal fusion for the treatment of Kienbock's disease: a three-dimensional theoretical study. J Orthop Res, 16(2), 256-263. doi: 10.1002/jor.1100160213

Kwak, S. D., Blankevoort, L., \& Ateshian, G. A. (2000). A Mathematical Formulation for 3D Quasi-Static Multibody Models of Diarthrodial Joints. Comput Methods Biomech Biomed Engin, 3(1), 41-64. doi: 10.1080/10255840008915253

Lemay, M. A., \& Crago, P. E. (1996). A dynamic model for simulating movements of the elbow, forearm, an wrist. J Biomech, 29(10), 1319-1330.

Li, G., Gil, J., Kanamori, A., \& Woo, S. L. (1999). A validated three-dimensional computational model of a human knee joint. J Biomech Eng, 121(6), 657-662.

Liacouras, P. C., \& Wayne, J. S. (2007). Computational modeling to predict mechanical function of joints: application to the lower leg with simulation of two cadaver studies. J Biomech Eng, 129(6), 811-817. doi: 10.1115/1.2800763

Lin, H. T., Nakamura, Y., Su, F. C., Hashimoto, J., Nobuhara, K., \& Chao, E. Y. (2005). Use of virtual, interactive, musculoskeletal system (VIMS) in modeling and analysis of shoulder throwing activity. J Biomech Eng, 127(3), 525-530. 
McGinley, J. C., \& Kozin, S. H. (2001). Interosseous membrane anatomy and functional mechanics. Clin Orthop Relat Res(383), 108-122.

Morey-Klapsing, G., Arampatzis, A., \& Bruggemann, G. P. (2005). Joint stabilising response to lateral and medial tilts. Clin Biomech (Bristol, Avon), 20(5), 517-525. doi: 10.1016/j.clinbiomech.2005.01.008

Morrey, B. F. (2000). The elbow and its disorders. N.B. Saunders. Independent square west. Philadelphia, PA.

Morrey, B. F., \& An, K. N. (1985). Functional anatomy of the ligaments of the elbow. Clin Orthop Relat Res(201), 84-90.

Morrey, B. F., \& Chao, E. Y. (1976). Passive motion of the elbow joint. J Bone Joint Surg Am, 58(4), 501-508.

Murray, W. M., Delp, S. L., \& Buchanan, T. S. (1995). Variation of muscle moment arms with elbow and forearm position. $J$ Biomech, 28(5), 513-525.

Nagano, A., Komura, T., Yoshioka, S., \& Fukashiro, S. (2005). Contribution of non-extensor muscles of the leg to maximal-effort countermovement jumping. Biomed Eng Online, 4, 52. doi: 10.1186/1475-925x-4-52

Netter, F. H., \& Hansen, J. T. (2003). Atlas of human anatomy (3rd ed.). Teterboro, N.J.: Icon Learning Systems.

Peck, C. C., Langenbach, G. E., \& Hannam, A. G. (2000). Dynamic simulation of muscle and articular properties during human wide jaw opening. Arch Oral Biol, 45(11), 963982.

Piazza, S. J., \& Delp, S. L. (2001). Three-dimensional dynamic simulation of total knee replacement motion during a step-up task. J Biomech Eng, 123(6), 599-606.

Pigeon, P., Yahia, L., \& Feldman, A. G. (1996). Moment arms and lengths of human upper limb muscles as functions of joint angles. J Biomech, 29(10), 1365-1370. 
Raikova, R. (1992). A general approach for modelling and mathematical investigation of the human upper limb. J Biomech, 25(8), 857-867.

Raikova, R. (1996). A model of the flexion-extension motion in the elbow joint some problems concerning muscle forces modelling and computation. J Biomech, 29(6), 763-772.

Regan, W. D., Korinek, S. L., Morrey, B. F., \& An, K. N. (1991). Biomechanical study of ligaments around the elbow joint. Clin Orthop Relat Res(271), 170-179.

Ring, D., \& Jupiter, J. B. (2000). Reconstruction of posttraumatic elbow instability. Clin Orthop Relat Res(370), 44-56.

Schuind, F., An, K. N., Berglund, L., Rey, R., Cooney, W. P., 3rd, Linscheid, R. L., \& Chao, E. Y. (1991). The distal radioulnar ligaments: a biomechanical study. J Hand Surg Am, 16(6), 1106-1114.

Shelburne, K. B., Pandy, M. G., Anderson, F. C., \& Torry, M. R. (2004). Pattern of anterior cruciate ligament force in normal walking. $J$ Biomech, 37(6), 797-805. doi: 10.1016/j.jbiomech.2003.10.010

Shiba, R., Sorbie, C., Siu, D. W., Bryant, J. T., Cooke, T. D., \& Wevers, H. W. (1988). Geometry of the humeroulnar joint. $J$ Orthop Res, 6(6), 897-906. doi: 10.1002/jor.1100060614

Skahen, J. R., 3rd, Palmer, A. K., Werner, F. W., \& Fortino, M. D. (1997). The interosseous membrane of the forearm: anatomy and function. J Hand Surg Am, 22(6), 981-985. doi: $10.1016 / \mathrm{s} 0363-5023(97) 80036-6$

Spratley, E. M., \& Wayne, J. S. (2011). Computational model of the human elbow and forearm: application to complex varus instability. Ann Biomed Eng, 39(3), 10841091. doi: 10.1007/s10439-010-0224-y

Stylianou, A. P., Guess, T. M., \& Cook, J. L. (2012). Development and validation of a multibody model of the canine stifle joint. Comput Methods Biomech Biomed Engin. doi: $10.1080 / 10255842.2012 .684243$ 
Tate, P. (2012). Seeley's Principles of anatomy \& physiology (2. ed.). New York, NY: McGraw-Hill.

Triolo, R. J., Werner, K. N., \& Kirsch, R. F. (2001). Modeling the postural disturbances caused by upper extremity movements. IEEE Trans Neural Syst Rehabil Eng, 9(2), 137-144. doi: 10.1109/7333.928573

van der Helm, F. C. (1994a). Analysis of the kinematic and dynamic behavior of the shoulder mechanism. J Biomech, 27(5), 527-550.

van der Helm, F. C. (1994b). A finite element musculoskeletal model of the shoulder mechanism. J Biomech, 27(5), 551-569.

Wiesel, S. W., \& Delahay, J. N. (2010). Essentials of Orthopedic Surgery: Springer.

Wismans, J., Veldpaus, F., Janssen, J., Huson, A., \& Struben, P. (1980). A three-dimensional mathematical model of the knee-joint. J Biomech, 13(8), 677-685.

Woo, S. L., Debski, R. E., Wong, E. K., Yagi, M., \& Tarinelli, D. (1999). Use of robotic technology for diathrodial joint research. J Sci Med Sport, 2(4), 283-297.

Wu, J. Z., Dong, R. G., Smutz, W. P., \& Schopper, A. W. (2003). Modeling of timedependent force response of fingertip to dynamic loading. J Biomech, 36(3), 383-392.

Zielinska, B., \& Donahue, T. L. (2006). 3D finite element model of meniscectomy: changes in joint contact behavior. J Biomech Eng, 128(1), 115-123. 


\section{VITA}

Md Munsur Rahman was born March 1, 1985 in Joypurhat, Bangladesh. He attended Kalai M.U. High school, a local public school and finished his tenth grade in 2001. The same year, he enrolled at the Kalai Degree College and graduated with "outstanding honors" in 2003. The following year, he entered in the Bangladesh University of Engineering and Technology (BUET) and began studying mechanical engineering. In the fall of 2008, he graduated with a Bachelor of Science degree in Mechanical Engineering.

After finishing the graduation, he started working with a famous refrigeration and air condition manufacturing company in Bangladesh. There, he had a chance to work closely with the manufacturing process and obtained a firsthand experience on industrial environment. After getting admission in University of Missouri-Kansas City in spring 2012 semester, he moved to the United States and started pursuing his M.S in Mechanical Engineering, with an emphasis in Biomechanics.

In January 2012, Munsur joined the Musculoskeletal Biomechanics Research Laboratory as a graduate research assistant and spent two full years working in the biomechanical lab under the direction of Dr. Trent Guess. Throughout his academic years, he gained considerable experience at developing a multi-body modeling of human elbow joints. Upon completion of his degree requirements for a Master of Science degree in Mechanical Engineering, Munsur plans to use his education to follow a career in biomechanics. 